\title{
On functoriality of Zelevinski involutions
}

\author{
Kaoru Hiraga
}

\begin{abstract}
In this paper, we present a conjecture on a relation between the Zelevinski involutions for reductive groups over a $p$-adic field and the conjectural $A$-packets. As evidence for this conjecture, we prove that the Zelevinski involutions, which are regarded as operators on the space of virtual characters, commute with the endoscopic transfers under the assumption of the fundamental lemma for groups and for Lie algebras.
\end{abstract}

\section{Introduction}

Let $F$ be a $p$-adic field and $G$ a connected reductive algebraic group defined over $F$. We denote by $W_{F}$ the Weil group of $F$. Let ${ }^{L} G=\hat{G} \rtimes W_{F}$ be the $L$-group of $G$. We denote by $\mathcal{L}^{G}$ the set of standard Levi subgroups of $G$. For $M \in \mathcal{L}^{G}$, we denote by $r(M)$ the semisimple split $F$-rank of $M$. Let $\Pi(G)$ be the set of equivalence classes of irreducible admissible representations of $G(F)$ and let $\mathbb{C}[\Pi(G)]$ be the space of virtual characters of $G(F)$. The parabolic induction defines a homomorphism $i_{M}^{G}: \mathbb{C}[\Pi(M)] \longrightarrow \mathbb{C}[\Pi(G)]$ and the (normalized) Jacquet functor defines a homomorphism $r_{M}^{G}: \mathbb{C}[\Pi(G)] \longrightarrow \mathbb{C}[\Pi(M)]$. Following Kato $\left[\right.$ Kat93], we define the Zelevinski involution $\mathbf{D}_{G}$ by

$$
\mathbf{D}_{G}=\sum_{M \in \mathcal{L}^{G}}(-1)^{r(M)} i_{M}^{G} \circ r_{M}^{G}
$$

Let $\{M\}$ be the set of associate standard Levi subgroups of $M$. We say that $\pi \in \Pi(G)$ is of type $\{M\}$ if $r_{M}^{G}(\pi)$ is a non-zero linear combination of supercuspidal representations of $M(F)$. If $\pi$ is of type $\{M\}$, then we put $r_{\pi}=r(M)$. For $\pi \in \Pi(G)$, we define

$$
\mathbf{d}_{G}(\pi)=(-1)^{r_{\pi}} \mathbf{D}_{G}(\pi) .
$$

Aubert [Aub95, Aub96] proved that $\mathbf{d}_{G}(\pi)$ is irreducible. Thus the Zelevinski involution preserves the irreducibility. It seems natural to consider the relation between the Zelevinski involution and the conjectural Langlands functoriality. Nevertheless, the Zelevinski involution does not preserve the $L$-packets. We consider the $A$-packets conjectured by Arthur [Art89, Conjecture 6.1]. (In this paper, we follow the formulation of [Art89, Conjecture 6.1], although we can find a modified conjecture due to Vogan in [Vog93]). For a Langlands parameter $\phi: W_{F} \times S U_{2}(\mathbb{C}) \longrightarrow{ }^{L} G$, we denote by $\Pi_{\phi}(G)$ the corresponding conjectural $L$-packet. Although $S U_{2}(\mathbb{C})$ is isomorphic to $S L_{2}(\mathbb{C})$, we denote the second factor of this group by $S U_{2}(\mathbb{C})$ in order to distinguish it from the factor $S L_{2}(\mathbb{C})$ used to define the Arthur parameters in [Art89]. Let

$$
\psi: W_{F} \times S U_{2}(\mathbb{C}) \times S L_{2}(\mathbb{C}) \longrightarrow{ }^{L} G
$$

be an Arthur parameter. We put

$$
\begin{gathered}
S_{\psi}=\operatorname{Cent}(\psi, \hat{G}), \\
\mathbb{S}_{\psi}=S_{\psi} / S_{\psi}^{0},
\end{gathered}
$$

Received 10 October 2002, accepted in final form 22 September 2003, published online 15 October 2004. 2000 Mathematics Subject Classification 22E50 (primary), 11F70, 22E35 (secondary).

Keywords: Zelevinski involution, functoriality, endoscopy.

This journal is (C) Foundation Compositio Mathematica 2004. 


\section{K. HiRAGA}

where $S_{\psi}^{0}$ is the identity component of $S_{\psi}$. Let $\Pi_{\psi}(G)$ be the conjectural $A$-packet of $\psi$ and $\rho=$ $\rho_{\psi}: S_{\psi} \longrightarrow \mathbb{C}^{\times}$the conjectural normalizing function. For $\bar{s} \in \mathbb{S}_{\psi}$ and $\pi \in \Pi_{\psi}(G)$, we define $\langle\bar{s}, \pi \mid \rho\rangle$ as in [Art89, Conjecture 6.1]. Then it is conjectured that

$$
\bar{s} \longrightarrow\langle\bar{s}, \pi \mid \rho\rangle
$$

is a virtual character of $\mathbb{S}_{\psi}$. We say that a virtual character $\theta \in \mathbb{C}[\Pi(G)]$ is stable if $\theta$ is stable as a distribution on $G(F)$. Let $\mathbb{C}[\Pi(G)]^{\text {st }}$ be the space of stable virtual characters of $G(F)$ and $\mathbb{C}\left[\Pi_{\psi}(G)\right]$ the subspace of $\mathbb{C}[\Pi(G)]$ generated by $\Pi_{\psi}(G)$. We put $\mathbb{C}\left[\Pi_{\psi}(G)\right]^{\text {st }}=\mathbb{C}[\Pi(G)]^{\text {st }} \cap \mathbb{C}\left[\Pi_{\psi}(G)\right]$.

In the case that $G$ is quasi-split, we put

$$
\mathbb{S}_{\psi}^{*}=S_{\psi} / S_{\psi}^{0} \cdot Z_{\hat{G}}^{\Gamma}
$$

where $Z_{\hat{G}}^{\Gamma}$ is the subgroup of the center $Z_{\hat{G}}$ of $\hat{G}$ consisting of the elements fixed by $\Gamma=\operatorname{Gal}(\bar{F} / F)$. We fix Whittaker data $\chi$ for $G$ (see $[\mathrm{KS} 99, \S 5.3]$ ). We determine the base point $\pi_{\chi} \in \Pi_{\phi_{\psi}}(G)$ as in $[\operatorname{Art} 89, \S 6]$, where $\Pi_{\phi_{\psi}}(G)$ is the $L$-packet corresponding to $\psi$. We define $\left\langle\bar{s}, \pi \mid \pi_{\chi}\right\rangle$ as in $[\operatorname{Art} 89$, Conjecture 6.1]. Then it is conjectured that $\left\langle\cdot, \pi \mid \pi_{\chi}\right\rangle$ is an irreducible character of $\mathbb{S}_{\psi}$. As $F$ is a $p$-adic field, the following hypothesis is believed.

Hypothesis 1.1. We have

$$
\operatorname{dim} \mathbb{C}\left[\Pi_{\psi}(G)\right]^{\mathrm{st}}=1 .
$$

In the following, we assume the Arthur conjecture [Art89, Conjecture 6.1] and Hypothesis 1.1.

Now we turn to the Zelevinski involution. We identify $S U_{2}(\mathbb{C})$ with $S L_{2}(\mathbb{C})$ and define $d(\psi)$ by

$$
d(\psi)(w \times t \times u)=\psi(w \times u \times t), \quad w \times t \times u \in W_{F} \times S U_{2}(\mathbb{C}) \times S L_{2}(\mathbb{C}) .
$$

Then $d(\psi)$ is the Arthur parameter constructed from $\psi$ by interchanging the role of $S U_{2}(\mathbb{C})$ and $S L_{2}(\mathbb{C})$.

Conjecture 1.2. We have

$$
\mathbf{d}_{G}\left(\Pi_{\psi}(G)\right)=\Pi_{d(\psi)}(G)
$$

Since $S_{\psi}=S_{d(\psi)}$, we may identify $\mathbb{S}_{\psi}$ with $\mathbb{S}_{d(\psi)}$. Let $\rho_{d}$ be the conjectural normalizing function of $d(\psi)$. In the case that $G$ is quasi-split, we denote the base point in $\Pi_{\phi_{d(\psi)}}(G)$ by $\pi_{d, \chi}$.

Conjecture 1.3. There exists a one-dimensional character $\mu$ of $\mathbb{S}_{\psi}$ such that

$$
\left\langle\bar{s}, \mathbf{d}_{G}(\pi) \mid \rho_{d}\right\rangle=\mu(\bar{s})\langle\bar{s}, \pi \mid \rho\rangle
$$

for all $\bar{s} \in \mathbb{S}_{\psi}$.

If $G$ is quasi-split, then the above formula is equal to

$$
\left\langle\bar{s}, \mathbf{d}_{G}(\pi) \mid \pi_{d, \chi}\right\rangle=\mu(\bar{s})\left\langle\bar{s}, \pi \mid \pi_{\chi}\right\rangle .
$$

(In the general case, the character $\mu$ may not be determined by the above relation.) The following conjecture is a special case of Conjecture 1.2.

Conjecture 1.4. If $G$ is quasi-split and if $\mathbb{S}_{\psi}^{*}=\{1\}$, then

$$
\mathbf{d}_{G}\left(\Pi_{\psi}(G)\right)=\Pi_{d(\psi)}(G) .
$$

As $F$ is a $p$-adic field, it is believed that the condition $G=G^{*}$ and $\mathbb{S}_{\psi}^{*}=\{1\}$ implies that $\Pi_{\psi}(G)=$ $\left\{\pi_{\chi}\right\}$ and $\Pi_{d(\psi)}(G)=\left\{\pi_{d, \chi}\right\}$. If we assume this, then Conjecture 1.4 asserts that $\mathbf{d}_{G}\left(\pi_{\chi}\right)=\pi_{d, \chi}$. In general, nevertheless, $\mathbf{d}_{G}\left(\pi_{\chi}\right)$ may not be equivalent to $\pi_{d, \chi}$. In fact, even in the case that $G=S L_{2}$, there exists $\psi$ such that $\mathbb{S}_{\psi}^{*} \neq\{1\}$ and that $\mathbf{d}_{G}\left(\pi_{\chi}\right) \neq \pi_{d, \chi}$ (see [LL79]). 


\section{ON FUNCTORIALITY OF ZELEVINSKI INVOLUTIONS}

In the case that $G=G L_{n}$, Conjecture 1.2 follows from the results of Moeglin and Waldspurger [MW86].

Recently, Takuya Konno and Kazuko Konno checked that Conjecture 1.2 is compatible with their candidates for the $A$-packets on the quasi-split unitary group in four variables in [Kon03].

Conjecture 1.3 implies that the Zelevinski involutions behave well under the endoscopic transfers. In this paper, we discuss the relation between the Zelevinski involutions and the endoscopic transfers. By Corollary 3.4, we have

$$
\mathbf{D}_{G}\left(\mathbb{C}[\Pi(G)]^{\text {st }}\right)=\mathbb{C}[\Pi(G)]^{\text {st }} .
$$

Let $(H, \mathcal{H}, s, \xi)$ be a set of (standard) endoscopic data. For the sake of brevity, we assume that $\mathcal{H} \cong{ }^{L} H$. Unfortunately the existence of the endoscopic transfer is still hypothetical. In this paper, we assume the fundamental lemma for groups [Art96, Hypothesis 3.1] and for Lie algebras [Wal97, Conjecture 1.3] to define the endoscopic transfer

$$
\operatorname{Tran}_{H}^{G}: \mathbb{C}[\Pi(H)]^{\text {st }} \longrightarrow \mathbb{C}[\Pi(G)]
$$

of virtual characters (see Proposition 4.6). Let $A_{0}$ be a maximal split torus of $G$ and let $A_{H, 0}$ be a maximal split torus of $H$. We put $a(G)=\operatorname{dim}\left(A_{0}\right)$ and $a(H)=\operatorname{dim}\left(A_{H, 0}\right)$. Then we have the following theorem (see Theorem 6.6).

Theorem 1.5. Assume the fundamental lemma for groups and for Lie algebras. Then we have

$$
\mathbf{D}_{G} \circ \operatorname{Tran}_{H}^{G}=(-1)^{a(G)-a(H)} \operatorname{Tran}_{H}^{G} \circ \mathbf{D}_{H} .
$$

(In the case that $\mathcal{H} \not{ }^{L} H$, we take a $z$-pair $\left(H_{1}, \xi_{H_{1}}\right)$ as in $[K S 99, \S 2.2]$; see the formula in Theorem 6.7.)

By using this theorem, we can reduce Conjecture 1.2 to Conjecture 1.4 (see Lemma 7.2). Moreover, if $G$ is quasi-split, then by using Theorem 1.5, we can show that Conjecture 1.4 implies the following formula (see Proposition 7.4):

$$
\left\langle\bar{s}, \mathbf{d}_{G}(\pi) \mid \pi_{d, \chi}\right\rangle=\left\langle\bar{s}, \mathbf{d}_{G}\left(\pi_{\chi}\right) \mid \pi_{d, \chi}\right\rangle\left\langle\bar{s}, \pi \mid \pi_{\chi}\right\rangle,
$$

where $\left\langle\cdot, \mathbf{d}_{G}\left(\pi_{\chi}\right) \mid \pi_{d, \chi}\right\rangle$ is a one-dimensional character of $\mathbb{S}_{\psi}$. This is Conjecture 1.3. In the case that $G$ is not quasi-split, Conjecture 1.4 implies the following formula:

$$
\langle\overline{1}, \pi \mid \rho\rangle=\left\langle\overline{1}, \mathbf{d}_{G}(\pi) \mid \rho_{d}\right\rangle .
$$

In the theory of endoscopy, some relations are defined modulo inner automorphisms. To avoid this ambiguity, we fix endoscopic data, an inner twisting and splittings in the following way. Let $\varphi: G \longrightarrow G^{*}$ be a quasi-split inner twisting of $G$ and $A_{0}^{*}$ a maximal split torus of $G^{*}$. We fix an $F$-splitting $\left(B_{0}^{*}, T_{0}^{*},\left\{X_{\alpha}\right\}\right)$ of $G^{*}$, an $F$-splitting $\left(B_{H, 0}, T_{H, 0},\left\{Y_{\alpha}\right\}\right)$ of $H$, a $\Gamma$-splitting $\left(\mathcal{B}, \mathcal{T},\left\{\mathcal{X}_{\check{\alpha}}\right\}\right)$ of $\hat{G}$ and a $\Gamma$-splitting $\left(\mathcal{B}_{H}, \mathcal{T}_{H},\left\{\mathcal{Y}_{\check{\alpha}}\right\}\right)$ of $\hat{H}$. Then we may identify $\hat{T}_{0}^{*}$ with $\mathcal{T}$ and $\hat{T}_{H, 0}$ with $\mathcal{T}_{H}$. We may assume that $A_{0}^{*} \subset T_{0}^{*}$ and that $A_{H, 0} \subset T_{H, 0}$. We say that a subtorus of $A_{0}^{*}$ is standard if it is equal to the split component of the center of a standard Levi subgroup of $G^{*}$. We assume that $s \in \mathcal{T}, \xi\left(\mathcal{T}_{H}\right)=\mathcal{T}$ and $\xi\left(\mathcal{B}_{H}\right) \subset \mathcal{B}$. Let $i_{0}^{*}: T_{H, 0} \longrightarrow T_{0}^{*}$ be the dual homomorphism of $\xi^{-1}: \mathcal{T} \longrightarrow \mathcal{T}_{H}$. We may assume that $i_{0}^{*}\left(A_{H, 0}\right)$ is a standard subtorus of $A_{0}^{*}$ (see $\S 4$ ). We choose an inner twisting $\varphi$ such that $\varphi\left(A_{0}\right)$ is a standard subtorus of $A_{0}^{*}$. We define a positive root system of $G$ by the pullback of the positive root system of $G^{*}$. For $M \in \mathcal{L}^{G}$, we have $\varphi(M) \in \mathcal{L}^{G^{*}}$.

In $\S 2$, we collect the properties of double cosets of Weyl groups with respect to endoscopic groups and standard Levi subgroups, which is a generalization of [Car93, $\S 2.7]$. The proofs of the results in $\S 2$ are contained in the Appendix. Assume that $G=G^{*}$. Let

$$
\begin{gathered}
\Omega(G)=\operatorname{Norm}\left(A_{0}, G\right) / \operatorname{Cent}\left(A_{0}, G\right), \\
\Omega(H)=\operatorname{Norm}\left(A_{H, 0}, H\right) / \operatorname{Cent}\left(A_{H, 0}, H\right),
\end{gathered}
$$




\section{K. HiRAGA}

be the Weyl groups. We denote the set of roots of $\left(G, A_{0}\right)$ by $R(G)=R\left(G, A_{0}\right)$ and the set of roots $\left(H, A_{H, 0}\right)$ by $R(H)=R\left(H, A_{H, 0}\right)$. We identify $A_{H, 0}$ with the image $i_{0}^{*}\left(A_{H, 0}\right)$ in $A_{0}=A_{0}^{*}$. By Lemma 4.3, we may regard $\Omega(H)$ as a subgroup of $\Omega(G)$. For $M \in \mathcal{L}^{G}$, we put

$$
\Omega(G)_{M, H}=\left\{\omega \in \Omega(G) \mid \omega\left(A_{H, 0}\right) \supset A_{M}\right\},
$$

where $A_{M}$ is the split component of the center of $M$. Let

$$
\tilde{D}_{M}=\left\{\omega \in \Omega(G)_{M, H}^{-1} \mid \omega\left(R^{+}(M)\right)>0\right\} .
$$

Then by Lemma 2.1, we can define $D_{M, H}$ by

$$
D_{M, H}=\left\{\omega \in \tilde{D}_{M}^{-1} \mid \omega\left(R^{+}(H)\right)>0\right\} .
$$

Proposition 2.2 asserts that $D_{M, H}$ is a system of representatives for

$$
\Omega(M) \backslash \Omega(G)_{M, H} / \Omega(H) .
$$

For $\omega \in D_{M, H}$, let $R=R(H) \cap \operatorname{res}_{A_{H, 0}}\left(\omega^{-1}(R(M))\right)$, then by Lemma $2.3, R$ is a root system of a standard Levi subgroup $M_{\omega}$ of $H$. For $L \in \mathcal{L}^{H}$, we put

$$
D_{M, H, L}=\left\{\omega \in D_{M, H} \mid M_{\omega}=L\right\}
$$

and

$$
a_{M, H, L}=\sharp D_{M, H, L} \text {. }
$$

Then we have the following formula, which is a generalization of [Car93, Proposition 2.7.7]:

$$
\sum_{M \in \mathcal{L}^{G}}(-1)^{r(M)} a_{M, H, L}=(-1)^{a(G)-a(H)} \cdot(-1)^{r(L)} .
$$

We turn to the general $G$. For $M \in \mathcal{L}^{G}$, let $M^{*}=\varphi(M)$ and $D_{M, H}=D_{M^{*}, H}$. For $\omega \in D_{M, H}$, we put $M_{\omega}=\left(M^{*}\right)_{\omega}$. Let ${ }^{L} M_{\omega}$ be the $L$-group of $M_{\omega}$. Then we may regard ${ }^{L} M_{\omega}$ as a standard Levi subgroup of ${ }^{L} H$. We choose a representative $\hat{n}_{\omega} \in \operatorname{Norm}(\mathcal{T}, \hat{G})$ for

$$
\omega \in \Omega\left(G^{*}\right) \subset \Omega\left(G^{*}, T_{0}^{*}\right) \cong \Omega(\hat{G}, \mathcal{T}) .
$$

We put $s_{\omega}^{\prime}=\operatorname{Int} \hat{n}_{\omega}(s)$ and $\xi_{\omega}=\operatorname{Int} \hat{n}_{\omega} \circ \xi$. Then Lemma 5.1 asserts that $\left(M_{\omega},{ }^{L} M_{\omega}, s_{\omega}^{\prime}, \xi_{\omega}\right)$ is a set of endoscopic data for $M$. The following formula in Theorem 5.6 is an analogue of the formula of Bernstein and Zelevinski [BZ77, Lemma 2.12]:

$$
r_{M}^{G} \circ \operatorname{Tran}_{H}^{G}=\sum_{\omega \in D_{M, H}} \operatorname{Tran}_{M_{\omega}}^{M} \circ r_{M_{\omega}}^{H} .
$$

Note that $D_{M, H}$ depends on the choice of a set of endoscopic data, an inner twisting and splittings.

In $\S \S 4$ and 5 , we prove this formula. In $\S 6$, we prove Theorem 1.5 by using (1.1) and (1.2). In $\S 7$, we discuss the conjectural relation between the Zelevinski involutions and the Arthur conjecture.

\section{Cosets of Weyl groups}

Properties of the double cosets of Weyl groups with respect to standard Levi subgroups are well known [Car93, § 2.7]. In this section, we collect results on the double cosets of Weyl groups with respect to endoscopic groups and standard Levi subgroups. Proofs of the results in this section are contained in the Appendix.

Let $\mathfrak{a}^{G}$ be a finite-dimensional vector space over the real number field $\mathbb{R}$ and let $\left(\mathfrak{a}^{G}\right)^{\prime}$ be the vector space of linear forms of $\mathfrak{a}^{G}$. In this paper, we allow a root system to have a center. Therefore, a subset $R \subset\left(\mathfrak{a}^{G}\right)^{\prime}$ is called a root system on $\mathfrak{a}^{G}$ if $R$ is a (non-reduced) root system in the vector space generated by $R$. Let $R(G)$ be a root system on $\mathfrak{a}^{G}$. We denote by $\Omega(G)$ the Weyl group of $R(G)$. 


\section{ON FUNCTORIALITY OF ZELEVINSKI INVOLUTIONS}

We fix a positive definite $\Omega(G)$-invariant symmetric bilinear form $($,$) on \left(\mathfrak{a}^{G}\right)^{\prime}$. This defines an isomorphism from $\left(\mathfrak{a}^{G}\right)^{\prime}$ to $\mathfrak{a}^{G}$ and a positive definite symmetric bilinear form on $\mathfrak{a}^{G}$. For $\tilde{\alpha} \in R(G)$, we denote the corresponding reflection on $\mathfrak{a}^{G}$ by $s_{\tilde{\alpha}}$. We fix a positive root system. We denote the set of positive roots by $R^{+}(G)$ and the set of negative roots by $R^{-}(G)$. The set of simple roots is denoted by $S(G)$. For a subset $S(M)$ of $S(G)$, we denote the corresponding subroot system by $R(M)$. We put $R^{+}(M)=R(M) \cap R^{+}(G)$ and

$$
\mathfrak{a}_{M}=\left\{a \in \mathfrak{a}^{G} \mid \tilde{\alpha}(a)=0 \text { for all } \tilde{\alpha} \in S(M)\right\} .
$$

Let $\Omega(M)$ be the Weyl group of $R(M)$. We have

$$
\begin{aligned}
& R(M)=\left\{\tilde{\alpha} \in R(G) \mid \tilde{\alpha}(a)=0 \text { for all } a \in \mathfrak{a}_{M}\right\}, \\
& \Omega(M)=\left\{\omega \in \Omega(G) \mid \omega(a)=a \text { for all } a \in \mathfrak{a}_{M}\right\} .
\end{aligned}
$$

We say that a subroot system $R \subset R(G)$ is standard if there exists $S(M) \subset S(G)$ such that $R=R(M)$ and a subspace $\mathfrak{a} \subset \mathfrak{a}^{G}$ is standard if there exists $S(M) \subset S(G)$ such that $\mathfrak{a}=\mathfrak{a}_{M}$. For a subspace $\mathfrak{a} \subset \mathfrak{a}^{G}$, we put

$$
\Omega(G)_{\mathfrak{a}}=\{\omega \in \Omega(G) \mid \omega(\mathfrak{a})=\mathfrak{a}\} .
$$

We denote the restriction of $\tilde{\alpha} \in R(G)$ to $\mathfrak{a}$ by $\operatorname{res}_{\mathfrak{a}}(\tilde{\alpha})$. If $\omega \in \Omega(G)_{\mathfrak{a}}$, then we denote the restriction of $\omega$ to $\mathfrak{a}$ by $\operatorname{res}_{\mathfrak{a}}(\omega)$. For a standard subspace $\mathfrak{a}_{M} \subset \mathfrak{a}^{G}$, we put

$$
R\left(G ; \mathfrak{a}_{M}\right)=\left\{\operatorname{res}_{\mathfrak{a}_{M}}(\tilde{\alpha}) \mid \tilde{\alpha} \in R(G)\right\}-\{0\} .
$$

For $\alpha \in R\left(G ; \mathfrak{a}_{M}\right)$, we denote by $s_{\alpha}$ the reflection on $\mathfrak{a}_{M}$ corresponding to $\alpha$ with respect to the restriction of the bilinear form $($,$) to \mathfrak{a}_{M}$. In general, $R\left(G ; \mathfrak{a}_{M}\right)$ may not be a root system on $\mathfrak{a}_{M}$. In this paper, we say that $\alpha \in R\left(G ; \mathfrak{a}_{M}\right)$ is $R(G)$-symmetric if there exists $\omega \in \Omega(G)_{\mathfrak{a}_{M}}$ such that $\operatorname{res}_{\mathfrak{a}_{M}}(\omega)=s_{\alpha}$. If there exists a simple root $\tilde{\alpha}$ such that $\alpha=\operatorname{res}_{\mathfrak{a}_{M}} \tilde{\alpha}$, then Lemma 4 asserts that $\alpha$ is $R(G)$-symmetric if and only if we have $\omega_{-}^{\alpha} \tilde{\alpha}=-\tilde{\alpha}$, where $\omega_{-}^{\alpha}$ is the longest element in the Weyl group of $S(M) \cup\{\tilde{\alpha}\}$. We denote by $R\left(G ; \mathfrak{a}_{M}\right)_{\text {sym }}$ the subset consisting of the $R(G)$-symmetric roots on $\mathfrak{a}_{M}$. We put $R^{+}\left(G ; \mathfrak{a}_{M}\right)=\left\{\operatorname{res}_{\mathfrak{a}_{M}}(\tilde{\alpha}) \mid \tilde{\alpha} \in R^{+}(G)\right\}-\{0\}$ and $R^{-}\left(G ; \mathfrak{a}_{M}\right)=-R^{+}\left(G ; \mathfrak{a}_{M}\right)$. Since $\mathfrak{a}_{M}$ is a standard subspace, the intersection of $R^{+}\left(G ; \mathfrak{a}_{M}\right)$ and $R^{-}\left(G ; \mathfrak{a}_{M}\right)$ are empty. We put $R^{+}\left(G ; \mathfrak{a}_{M}\right)_{\text {sym }}=R^{+}\left(G ; \mathfrak{a}_{M}\right) \cap R\left(G ; \mathfrak{a}_{M}\right)_{\text {sym }}$. We write $\tilde{\alpha}>0$ if $\tilde{\alpha}$ is a positive root and $\tilde{\alpha}<0$ if $\tilde{\alpha}$ is a negative root. Moreover, for a subset $R \subset R(G)$, we write $R>0$ if $R \subset R^{+}(G)$ and $R<0$ if $R \subset R^{-}(G)$.

Until the end of this section, we fix $S\left(M^{H}\right) \subset S(G)$. We put $\mathfrak{a}^{H}=\mathfrak{a}_{M^{H}}$. We abbreviate res $\mathfrak{a}^{H}$ to $\operatorname{res}_{H}$ and $\Omega(G)_{\mathfrak{a}^{H}}$ to $\Omega(G)_{H}$. We also fix a root system $R(H) \subset R\left(G ; \mathfrak{a}^{H}\right)_{\text {sym }}$ on $\mathfrak{a}^{H}$. Let $R^{+}(H)=$ $R(H) \cap R^{+}\left(G ; \mathfrak{a}^{H}\right)_{\text {sym }}$, then $R^{+}(H)$ is a positive system of $R(H)$. For $S(M) \subset S(G)$ and $\omega \in \Omega(G)$, we put

$$
l_{M}(\omega)=\sharp\left\{\tilde{\alpha} \in R^{+}(M) \mid \omega \tilde{\alpha}<0\right\} .
$$

We denote by $\Omega(H)$ the Weyl group of $R(H)$ acting on $\mathfrak{a}^{H}$. Since $\alpha \in R(H)$ is $R(G)$-symmetric, there exists a unique $\tilde{s}_{\alpha} \in \Omega(G)_{H}$ such that $\operatorname{res}_{H} \tilde{s}_{\alpha}=s_{\alpha}$ and $l_{M^{H}}\left(\tilde{s}_{\alpha}\right)=0$. Therefore, for each $\omega \in \Omega(H)$, there exists a unique $\tilde{\omega} \in \Omega(G)_{H}$ such that $\operatorname{res}_{H}(\tilde{\omega})=\omega$ and $l_{M^{H}}(\tilde{\omega})=0$. The homomorphism $\omega \longrightarrow \tilde{\omega}$ allows us to regard $\Omega(H)$ as a subgroup of $\Omega(G)$. We put

$$
\tilde{D}_{M}=\left\{\omega \in \Omega(G) \mid l_{M}(\omega)=0 \text { and } \omega\left(\mathfrak{a}_{M}\right) \subset \mathfrak{a}^{H}\right\} .
$$

We write $\mathbb{R}_{+}^{\times}$for $\{x \in \mathbb{R} \mid x>0\}$.

Lemma 2.1. Let $\omega \in \tilde{D}_{M}^{-1}$ and $\omega^{\prime} \in \Omega(G)_{H}$. Let $\tilde{\alpha} \in R^{+}(G)$ be a positive root satisfying $\operatorname{res}_{H}(\tilde{\alpha}) \neq 0$. If $\omega \omega^{\prime} \tilde{\alpha}>0$, then for any $\tilde{\alpha}^{\prime} \in R^{+}(G)$ satisfying $\operatorname{res}_{H}\left(\tilde{\alpha}^{\prime}\right) \in \mathbb{R}_{+}^{\times} \operatorname{res}_{H}(\tilde{\alpha})$, we have $\omega \omega^{\prime} \tilde{\alpha}^{\prime}>0$ and if $\omega \omega^{\prime} \tilde{\alpha}<0$, then for any $\tilde{\alpha}^{\prime} \in R^{+}(G)$ satisfying $\operatorname{res}_{H}\left(\tilde{\alpha}^{\prime}\right) \in \mathbb{R}_{+}^{\times} \operatorname{res}_{H}(\tilde{\alpha})$, we have $\omega \omega^{\prime} \tilde{\alpha}^{\prime}<0$. 


\section{K. HiRAGA}

Let $\omega \in \tilde{D}_{M}^{-1}$ and $\alpha \in R^{+}\left(G ; \mathfrak{a}^{H}\right)$, then we say that $\omega \alpha$ is positive if we have $\omega \tilde{\alpha}>0$ for all $\tilde{\alpha} \in R^{+}(G)$ satisfying $\operatorname{res}_{H}(\tilde{\alpha}) \in \mathbb{R}_{+}^{\times} \alpha$ and $\omega \alpha$ is negative if we have $\omega \tilde{\alpha}<0$ for all $\tilde{\alpha} \in R^{+}(G)$ satisfying $\operatorname{res}_{H}(\tilde{\alpha}) \in \mathbb{R}_{+}^{\times} \alpha$. We write $\omega \alpha>0$ if $\omega \alpha$ is positive and $\omega \alpha<0$ if $\omega \alpha$ is negative. For $\omega \in \tilde{D}_{M}^{-1}$, we define $l_{H}(\omega)$ by

$$
l_{H}(\omega)=\sharp\left\{\alpha \in R^{+}(H) \mid \omega \alpha<0\right\} .
$$

We put

$$
D_{M, H}=\left\{\omega \in \tilde{D}_{M}^{-1} \mid l_{H}(\omega)=0\right\}
$$

We also put

$$
\Omega(G)_{M, H}=\left\{\omega \in \Omega(G) \mid \omega\left(\mathfrak{a}^{H}\right) \supset \mathfrak{a}_{M}\right\},
$$

then $\Omega(G)_{M, H}$ is invariant under the left and right action of $\Omega(M), \Omega(H)$, respectively.

Proposition 2.2. The subset $D_{M, H} \subset \Omega(G)_{M, H}$ is a system of representatives for the set of double cosets $\Omega(M) \backslash \Omega(G)_{M, H} / \Omega(H)$.

The set of standard subroot systems $\{R(M) \mid S(M) \subset S(G)\}$ of $R(G)$ is denoted by $\mathcal{L}^{G}$. We write $\mathcal{L}^{H}$ for the set of standard subroot systems of $R(H)$.

Lemma 2.3. Let $\omega \in D_{M, H}$, then

$$
R(H) \cap \operatorname{res}_{H}\left(\omega^{-1}(R(M))\right) \in \mathcal{L}^{H} .
$$

For $R(M) \in \mathcal{L}^{G}$ and $R(L) \in \mathcal{L}^{H}$, we put

$$
\begin{aligned}
D_{M, H, L} & =\left\{\omega \in D_{M, H} \mid R(H) \cap \operatorname{res}_{H}\left(\omega^{-1}(R(M))\right)=R(L)\right\}, \\
a_{M, H, L} & =\sharp D_{M, H, L} .
\end{aligned}
$$

We write $r(M)$ for $\sharp S(M)$ and $r(L)$ for $\sharp S(L)$. The following theorem is the main result of this section.

TheOREM 2.4. We have

$$
\sum_{R(M) \in \mathcal{L}^{G}}(-1)^{r(M)} a_{M, H, L}=(-1)^{r\left(M^{H}\right)} \cdot(-1)^{r(L)} .
$$

The following lemma will be used in $\S 6$.

Lemma 2.5. Let $R(M), R\left(M_{0}\right) \in \mathcal{L}^{G}$. Assume that $R\left(M_{0}\right)$ has no other associate standard subroot system than $R\left(M_{0}\right)$ itself. If $R(M)$ satisfies $\omega\left(\mathfrak{a}_{M}\right) \subset \mathfrak{a}_{M_{0}}$ for some $\omega \in \Omega(G)$, then we have

$$
R(M) \supset R\left(M_{0}\right) .
$$

\section{Zelevinski involutions}

Let $G$ be a connected reductive linear algebraic group defined over a $p$-adic field $F$. We denote by $\Gamma$ the Galois group $\operatorname{Gal}(\bar{F} / F)$ of $F$. We fix a minimal parabolic subgroup $P_{0}$ of $G$ defined over $F$. We also fix a Levi subgroup $M_{0} \subset P_{0}$ defined over $F$ and a maximal torus $T_{0} \subset M_{0}$ defined over $F$. We denote by $A_{0}$ the split component of $T_{0}$. Let $G^{*}$ be a quasi-split inner form of $G$ and let $\varphi: G \longrightarrow G^{*}$ be an inner twisting. We fix a Borel subgroup $B_{0}^{*}$ of $G^{*}$ defined over $F$ and a maximal torus $T_{0}^{*} \subset B_{0}^{*}$ defined over $F$. Let $R\left(G^{*}, T_{0}^{*}\right)$ be the set of roots of $\left(G^{*}, T_{0}^{*}\right)$, $R^{+}\left(G^{*}, T_{0}^{*}\right)$ the set of positive roots corresponding to $B_{0}^{*}$ and $S\left(G^{*}, T_{0}^{*}\right)$ the set of simple roots. We write $\mathcal{L}^{G^{*}}$ for the set of standard Levi subgroups of $G^{*}$. Let $A_{0}^{*}$ be the split component of $T_{0}^{*}$. 


\section{ON FUNCTORIALITY OF ZELEVINSKI INVOLUTIONS}

For $M^{*} \in \mathcal{L}^{G^{*}}$, we denote by $A_{M^{*}}$ the split component of the center $Z_{M^{*}}$ of $M^{*}$. We say that a subtorus $A \subset A_{0}^{*}$ is standard if there exists a standard Levi subgroup $M^{*} \in \mathcal{L}^{G^{*}}$ such that $A=A_{M^{*}}$. For a subtorus $A \subset A_{0}^{*}$, we put $M_{A}^{*}=\operatorname{Cent}\left(A, G^{*}\right)$. We may assume that $\varphi$ satisfies $\varphi\left(T_{0}\right)=T_{0}^{*}$ and that $\varphi\left(A_{0}\right)$ is a standard subtorus of $A_{0}^{*}$. Moreover, we assume that $\varphi\left(P_{0}\right) \supset B_{0}^{*}$. Therefore, we can define a set of positive roots $R^{+}\left(G, T_{0}\right)$ by the pullback of $R^{+}\left(G^{*}, T_{0}^{*}\right)$. Let $\mathcal{L}^{G}$ be the set of standard Levi subgroups of $G$, then for any $M \in \mathcal{L}^{G}$, we have $\varphi(M) \in \mathcal{L}^{G^{*}}$. Moreover, it is easy to see that the restriction of $\varphi$ to $M$ is an inner twisting of $M$ to the quasi-split inner form $\varphi(M)$. We put $r(M)=\sharp S\left(M, A_{0}\right)$. We denote by $\Omega\left(G, T_{0}\right)$ the Weyl group of $\left(G, T_{0}\right)$ and by $\Omega\left(G, T_{0}\right)_{F}$ the subgroup consisting of the elements defined over $F$. We write $R\left(G, A_{0}\right)$ for the set of relative roots of $\left(G, A_{0}\right)$. We define the set of positive roots $R^{+}\left(G, A_{0}\right)$ by the restriction of $R^{+}\left(G, T_{0}\right)$. Let $S\left(G, A_{0}\right)$ be the set of simple roots and $\Omega\left(G, A_{0}\right)$ the Weyl group of $\left(G, A_{0}\right)$. We define $R\left(G^{*}, A_{0}^{*}\right), R^{+}\left(G^{*}, A_{0}^{*}\right), S\left(G^{*}, A_{0}^{*}\right), \Omega\left(G^{*}, A_{0}^{*}\right)$ and $r\left(M^{*}\right)$ similarly. For a maximal torus $T \subset G$ defined over $F$, we denote by $A_{T}$ the split component of $T$. Therefore, $A_{T}$ is conjugate to a standard subtorus of $A_{0}$. Let $\Omega(G(F), T)=\operatorname{Norm}(T, G(F)) / T(F)$, then $\Omega(G(F), T)$ is a subgroup of $\Omega(G, T)_{F}$. Since $G^{*}$ is quasi-split, we have

$$
\Omega\left(G^{*}(F), T_{0}^{*}\right)=\Omega\left(G^{*}, T_{0}^{*}\right)_{F} \cong \Omega\left(G^{*}, A_{0}^{*}\right) .
$$

We identify $\Omega\left(G^{*}, A_{0}^{*}\right)$ with $\Omega\left(G^{*}, T_{0}^{*}\right)_{F}$. We determine a Haar measure on $T(F)$ by the condition that the volume of the maximal compact subgroup of $T(F)$ is 1 . Let $G_{\text {reg }}$ be the set of strongly regular semisimple elements in $G(F)$ and $G_{\text {ell }}$ the set of elliptic elements in $G_{\text {reg }}$. We denote by $\Pi(G)$ the set of equivalence classes of irreducible admissible representations of $G(F)$. We write $\mathbb{C}[\Pi(G)]$ for the space of virtual characters. Then $\mathbb{C}[\Pi(G)]$ consists of the finite linear combinations of $\Pi(G)$. For $M \in \mathcal{L}^{G}$, let

$$
i_{M}^{G}: \mathbb{C}[\Pi(M)] \longrightarrow \mathbb{C}[\Pi(G)]
$$

be the homomorphism corresponding to the (normalized) induction and

$$
r_{M}^{G}: \mathbb{C}[\Pi(G)] \longrightarrow \mathbb{C}[\Pi(M)]
$$

the homomorphism corresponding to the (normalized) Jacquet functor. Let $\pi \in \Pi(G)$, then by the theorem of Harish-Chandra [Har78], the distribution character of $\pi$ can be represented by a locally constant function $\mathrm{ch}_{\pi}$ on $G_{\text {reg. }}$. We define a function $I^{G}(\pi)$ on $G_{\text {reg }}$ by

$$
I^{G}(\pi, \gamma)=\Delta_{G}(\gamma) \operatorname{ch}_{\pi}(\gamma)
$$

where $\gamma \in G_{\text {reg }}$ and $\Delta_{G}(\gamma)=\left|\prod_{\alpha}(\alpha(\gamma)-1)\right|_{F}^{1 / 2}$ is the Weyl denominator. We extend this definition to $\theta \in \mathbb{C}[\Pi(G)]$. We denote by $C_{c}^{\infty}(G)$ the space of locally constant compactly supported functions on $G(F)$. For $f \in C_{c}^{\infty}(G)$, we define

$$
I(\gamma, f)=\Delta_{G}(\gamma) \int_{G(F) / G_{\gamma}(F)} f\left(g \gamma g^{-1}\right) d g,
$$

where $\gamma \in G_{\text {reg }}$ and $G_{\gamma}=\operatorname{Cent}(\gamma, G)$. Let $\{\gamma\}_{\text {st }}$ be the set of conjugacy classes in the stable conjugacy class of $\gamma$. We define

$$
I^{\mathrm{st}}(\gamma, f)=\sum_{\gamma^{\prime} \in\{\gamma\}_{\mathrm{st}}} I\left(\gamma^{\prime}, f\right) .
$$

Put $C_{c}^{\infty}(G)^{-}=\left\{f \in C_{c}^{\infty}(G) \mid I^{\text {st }}(\gamma, f)=0\right.$ for all $\left.\gamma \in G_{\text {reg }}\right\}$. We say that a distribution $D$ on $G(F)$ is stable if $D$ satisfies $D(f)=0$ for all $f \in C_{c}^{\infty}(G)^{-}$. We also say that a virtual character $\theta \in \mathbb{C}[\Pi(G)]$ is stable if $\theta$ is stable as a distribution on $G(F)$. Then $\theta$ is stable if and only if $I^{G}(\theta, \gamma)=I^{G}\left(\theta, \gamma^{\prime}\right)$ holds for all $\gamma \in G_{\text {reg }}$ and $\gamma^{\prime} \in\{\gamma\}_{\text {st }}$. We denote by $\mathbb{C}[\Pi(G)]^{\text {st }}$ the subspace of stable virtual characters. For a maximal torus $T^{M}$ of $M$ and a maximal torus $T^{G}$ of $G$, we put

$$
I_{\text {conj }}^{G, M}\left(T^{M}, T^{G}\right)=\left\{i: T^{M} \longrightarrow T^{G} \mid i=\operatorname{Int} g \text { for some } g \in G(F)\right\} .
$$




\section{K. Hiraga}

Then $\Omega\left(G(F), T^{G}\right)$ and $\Omega\left(M(F), T^{M}\right)$ act on $I_{\text {conj }}^{G, M}\left(T^{M}, T^{G}\right)$ from the left and right, respectively. Let $\mathcal{T}_{\text {conj }}^{G}$ be a system of representatives for the conjugacy classes of maximal tori of $G$. We choose a Haar measure on $M(F)$ in such a way that the following formula holds.

$$
I^{G}\left(i_{M}^{G}(\theta), \gamma\right)=\sum_{T^{M} \in \mathcal{T}_{\text {conj }}^{M}} \sum_{i \in I_{\text {conj }}^{G, M}\left(T^{M}, G_{\gamma}\right) / \Omega\left(M(F), T^{M}\right)} I^{M}\left(\theta, i^{-1}(\gamma)\right), \quad \gamma \in G_{\text {reg. }} .
$$

We put $M_{G \text {-reg }}=M(F) \cap G_{\text {reg. }}$. We denote by $A_{M}^{-}$the set of $a \in A_{M}(F)$ satisfying $|\alpha(a)|_{F}<1$ for all $\alpha \in R^{+}\left(G, A_{0}\right)-R^{+}\left(M, A_{0}\right)$. In this paper, we use Casselman's character formula in the following form.

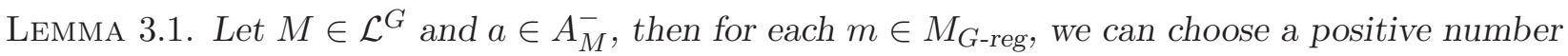
$n_{0}$ such that

$$
I^{G}\left(\theta, a^{n} m\right)=I^{M}\left(r_{M}^{G}(\theta), a^{n} m\right)
$$

holds for any $\theta \in \mathbb{C}[\Pi(G)]$ and $n \geqslant n_{0}$.

Proof. Let $P$ be the standard parabolic subgroup with Levi factor $M$. For each $m \in M_{G \text {-reg }}$ we can choose an $M$-conjugate $m^{\prime} \in M(F)$ and a positive number $n_{0}$ so that for all $n \geqslant n_{0}$ the parabolic subgroup $P_{a^{n} m^{\prime}}$ in [Cas77] is a standard parabolic subgroup contained in $P$ and $a^{n} m^{\prime} \in G_{\text {reg. }}$. Let $L \in \mathcal{L}^{G}$ be the standard Levi subgroup of $P_{a^{n} m^{\prime}}$. Then by [Cas77, Theorem 5.2], we have $I^{G}\left(\theta, a^{n} m^{\prime}\right)=I^{L}\left(r_{L}^{G}(\theta), a^{n} m^{\prime}\right)$ for all $n \geqslant n_{0}$ and $\theta \in \mathbb{C}[\Pi(G)]$. On the other hand, by applying [Cas77, Theorem 5.2] to $r_{M}^{G}(\theta)$, we have $I^{M}\left(r_{M}^{G}(\theta), a^{n} m^{\prime}\right)=I^{L}\left(r_{L}^{M} \circ r_{M}^{G}(\theta), a^{n} m^{\prime}\right)$. Thus $I^{G}\left(\theta, a^{n} m^{\prime}\right)=I^{M}\left(r_{M}^{G}(\theta), a^{n} m^{\prime}\right)$. Since $a^{n} m$ and $a^{n} m^{\prime}$ are $M$-conjugate, this completes the proof of the lemma.

Let $\theta \in \mathbb{C}[\Pi(M)], m \in M_{\text {reg }}$ and $a \in A_{M}$, then

$$
n \in \mathbb{Z} \longrightarrow I^{M}\left(\theta, a^{n} m\right)
$$

is a finite linear combination of quasi-characters of $\mathbb{Z}$. Hence, we have the following lemma.

Lemma 3.2. Let $\theta, \theta^{\prime} \in \mathbb{C}[\Pi(M)], m, m^{\prime} \in M_{\text {reg }}$ and $a, a^{\prime} \in A_{M}$. If there exists a positive number $n_{0}$ such that

$$
I^{M}\left(\theta, a^{n} m\right)=I^{M}\left(\theta^{\prime}, a^{\prime n} m^{\prime}\right)
$$

holds for all $n \geqslant n_{0}$, then we have

$$
I^{M}(\theta, m)=I^{M}\left(\theta^{\prime}, m^{\prime}\right) .
$$

Lemma 3.3. We have

$$
\begin{aligned}
& i_{M}^{G}\left(\mathbb{C}[\Pi(M)]^{\mathrm{st}}\right) \subset \mathbb{C}[\Pi(G)]^{\mathrm{st}}, \\
& r_{M}^{G}\left(\mathbb{C}[\Pi(G)]^{\mathrm{st}}\right) \subset \mathbb{C}[\Pi(M)]^{\mathrm{st}} .
\end{aligned}
$$

Proof. Let $m, m^{\prime} \in M_{G \text {-reg }}, \theta \in \mathbb{C}[\Pi(G)]^{\text {st }}$ and $a \in A_{M}^{-}$. Suppose that $m$ and $m^{\prime}$ are stably $M$-conjugate. Then $a^{n} m$ and $a^{n} m^{\prime}$ are stably $G$-conjugate. Therefore, by using Lemma 3.1, we have $I^{M}\left(r_{M}^{G}(\theta), a^{n} m\right)=I^{M}\left(r_{M}^{G}(\theta), a^{n} m^{\prime}\right)$ for sufficiently large $n$. Hence, by Lemma 3.2, we have the required relation for $r_{M}^{G}$. The relation for $i_{M}^{G}$ is well known.

We define the Zelevinski involution $\mathbf{D}_{G}$ by

$$
\mathbf{D}_{G}=\sum_{M \in \mathcal{L}^{G}}(-1)^{r(M)} i_{M}^{G} \circ r_{M}^{G} .
$$




\section{ON FUNCTORIALITY OF ZELEVINSKI INVOLUTIONS}

(See [Kat93] and [Aub95].) It is known that $\mathbf{D}_{G} \circ \mathbf{D}_{G}=$ id and that $\mathbf{D}_{G} \circ i_{M}^{G}=i_{M}^{G} \circ \mathbf{D}_{M}$. If $\pi \in \Pi(G)$, then Aubert [Aub95, Aub96] has shown that either $\mathbf{D}_{G}(\pi)$ or $-\mathbf{D}_{G}(\pi)$ is irreducible. Now, Lemma 3.3 implies the following corollary.

Corollary 3.4. We have

$$
\mathbf{D}_{G}\left(\mathbb{C}[\Pi(G)]^{\mathrm{st}}\right)=\mathbb{C}[\Pi(G)]^{\mathrm{st}} .
$$

\section{Endoscopy}

We denote by $W_{F}$ the Weil group of $F$. We write $\hat{G}$ for the dual group of $G$ and write ${ }^{L} G$ for the $L$-group $\hat{G} \rtimes W_{F}$ of $G$. We fix a $\Gamma$-splitting $\left(\mathcal{B}, \mathcal{T},\left\{\mathcal{X}_{\check{\alpha}}\right\}\right)$ of $\hat{G}$. We also fix an $F$-splitting $\left(B_{0}^{*}, T_{0}^{*},\left\{X_{\alpha}\right\}\right)$ containing the pair $\left(B_{0}^{*}, T_{0}^{*}\right)$. Then we may identify $\mathcal{T}$ with the dual group $\hat{T}_{0}^{*}$ of $T_{0}^{*}$. Let $(H, \mathcal{H}, s, \xi)$ be a set of endoscopic data for $G$. In this paper, we say that $(H, \mathcal{H}, s, \xi)$ and $\left(H^{\prime}, \mathcal{H}^{\prime}, s^{\prime}, \xi^{\prime}\right)$ are equivalent endoscopic data if there exists $g \in \hat{G}$ such that $g \xi(\mathcal{H}) g^{-1}=\xi^{\prime}\left(\mathcal{H}^{\prime}\right)$ and $\mathrm{gsg}^{-1} \in s^{\prime} \cdot Z_{\hat{G}}$. We fix an $F$-splitting $\left(B_{H, 0}, T_{H, 0},\left\{Y_{\alpha}\right\}\right)$ of $H$ and a $\Gamma$-splitting $\left(\mathcal{B}_{H}, \mathcal{T}_{H},\left\{\mathcal{Y}_{\check{\alpha}}\right\}\right)$ of $\hat{H}$. We identify $\mathcal{T}_{H}$ with $\hat{T}_{H, 0}$. By replacing the endoscopic data by equivalent data, we may assume that $s \in \mathcal{T}, \xi\left(\mathcal{T}_{H}\right)=\mathcal{T}$ and $\xi\left(\mathcal{B}_{H}\right) \subset \mathcal{B}$. Let

$$
i_{0}^{*}: T_{H, 0} \longrightarrow T_{0}^{*}
$$

be the dual homomorphism of

$$
\xi^{-1}: \hat{T}_{0}^{*} \cong \mathcal{T} \longrightarrow \mathcal{T}_{H} \cong \hat{T}_{H, 0} .
$$

We also write $i_{0}^{*}$ for the corresponding morphism $R\left(H, T_{H, 0}\right) \longrightarrow R\left(G, T_{0}^{*}\right)$. We denote by $A_{H, 0}$ the split component of $T_{H, 0}$.

Lemma 4.1. There exists $\omega \in \Omega\left(G^{*}, T_{0}^{*}\right)$ such that $\omega \circ i_{0}^{*}\left(A_{H, 0}\right)$ is a standard subtorus of $A_{0}^{*}$ and that

$$
\omega \circ i_{0}^{*}\left(R^{+}\left(H, T_{H, 0}\right)\right) \subset R^{+}\left(G^{*}, T_{0}^{*}\right) .
$$

Proof. Choose an admissible embedding Int $g \circ i_{0}^{*}: T_{H, 0} \longrightarrow T^{*}=\operatorname{Int} g\left(T_{0}^{*}\right)$ of $T_{H, 0}$, where $g \in$ $G^{*}(\bar{F})$. We may assume that $A_{T^{*}}$ is a standard subtorus of $A_{0}^{*}$. Put $M^{*}=M_{A_{T^{*}}}$. Then there exists $m \in M^{*}(\bar{F})$ such that $m g \in \operatorname{Norm}\left(T_{0}^{*}, G^{*}\right)$. Let $\omega^{\prime} \in \Omega\left(G^{*}, T_{0}^{*}\right)$ be the image of $m g$. Put

$$
R^{\prime+}\left(H, T_{H, 0}\right)=\left\{\alpha \in R\left(H, T_{H, 0}\right) \mid \omega^{\prime} \circ i_{0}^{*}(\alpha) \in R^{+}\left(G^{*}, T_{0}^{*}\right)\right\} .
$$

Then $R^{\prime+}\left(H, T_{H, 0}\right)$ is preserved by $\Gamma$. Therefore, there exists an element $\omega_{H} \in \Omega\left(H, T_{H, 0}\right)_{F}$ such that $\omega_{H}\left(R^{+}\left(H, T_{H, 0}\right)\right)=R^{\prime+}\left(H, T_{H, 0}\right)$. Since $i_{0}^{*} \circ \Omega\left(H, T_{H, 0}\right) \circ i_{0}^{*-1} \subset \Omega\left(G^{*}, T_{0}^{*}\right)$, we have $\omega^{\prime} \cdot\left(i_{0}^{*} \circ\right.$ $\left.\omega_{H} \circ i_{0}^{*-1}\right) \in \Omega\left(G^{*}, T_{0}^{*}\right)$. Put $\omega=\omega^{\prime} \cdot\left(i_{0}^{*} \circ \omega_{H} \circ i_{0}^{*-1}\right)$. Then $\omega$ satisfies the required properties.

We identify $\Omega\left(G^{*}, T_{0}^{*}\right)$ with $\Omega(\hat{G}, \mathcal{T})$. We also identify $\Omega\left(H, T_{H}\right)$ with $\Omega\left(\hat{H}, \mathcal{T}_{H}\right)$. Let $\hat{n}_{\omega} \in$ $\operatorname{Norm}(\mathcal{T}, \hat{G})$ be a representative for $\omega \in \Omega(\hat{G}, \mathcal{T})$. Since $(H, \mathcal{H}, s, \xi)$ and $\left(H, \mathcal{H}, \operatorname{Int} \hat{n}_{\omega}(s)\right.$, Int $\left.\hat{n}_{\omega} \circ \xi\right)$ are equivalent endoscopic data, we may assume that $i_{0}^{*}\left(A_{H, 0}\right)$ is a standard subtorus of $A_{0}^{*}$ and that

$$
\begin{gathered}
s \in \mathcal{T}, \\
\xi\left(\mathcal{T}_{H}\right)=\mathcal{T}, \\
\xi\left(\mathcal{B}_{H}\right) \subset \mathcal{B} .
\end{gathered}
$$

We put $A^{H}=i_{0}^{*}\left(A_{H, 0}\right)$ and $M^{H}=\operatorname{Cent}\left(A^{H}, G^{*}\right)$. Then $M^{H} \in \mathcal{L}^{G^{*}}$.

Lemma 4.2. Let $A_{1}$ and $A_{2}$ be subtori of $A_{0}^{*}$. If $g \in G^{*}(\bar{F})$ satisfies $\operatorname{Int} g\left(A_{1}\right)=A_{2}$, then there exists $\omega \in \Omega\left(G^{*}, T_{0}^{*}\right)_{F}$ such that $\omega\left(A_{1}\right)=A_{2}$ and $\operatorname{res}_{A_{1}}\left(\omega^{-1} \circ \operatorname{Int} g\right)=\operatorname{id}_{A_{1}}$.

Proof. Put $M_{2}^{*}=\operatorname{Cent}\left(A_{2}, G^{*}\right)$. Then $M_{2}^{*}$ is quasi-split and $T_{0}^{*}$ is a maximally split torus of $M_{2}^{*}$. Since $A_{1}$ and $A_{2}$ are split tori, we have $\operatorname{res}_{A_{2}}\left(\operatorname{Int} \sigma(g) g^{-1}\right)=\operatorname{id}_{A_{2}}$ for any $\sigma \in \Gamma$. This implies 


\section{K. HiRAGA}

that $\sigma(g) g^{-1} \in M_{2}^{*}(\bar{F})$. Hence, by [Kot82, Corollary 2.2], there exists $m \in M_{2}^{*}(\bar{F})$ such that the torus $T^{*}=\operatorname{Int} m g\left(T_{0}^{*}\right)$ is defined over $F$ and that the homomorphism Int $m g: T_{0}^{*} \longrightarrow T^{*}$ is defined over $F$. This implies that $T^{*}$ is also a maximally split torus of $M_{2}^{*}$. Therefore, we may assume that $T^{*}=T_{0}^{*}$. Then $m g \in \operatorname{Norm}\left(T_{0}^{*}, G^{*}\right)$. Let $\omega \in \Omega\left(G^{*}, T_{0}^{*}\right)_{F}$ be the image of $m g$, then it is immediate that $\omega\left(A_{1}\right)=A_{2}$ and $\operatorname{res}_{A_{1}}\left(\omega^{-1} \circ \operatorname{Int} g\right)=\operatorname{id}_{A_{1}}$.

Lemma 4.3. Let $\omega \in \Omega\left(H, A_{H, 0}\right)$, then there exists a unique $\omega_{G} \in \Omega\left(G^{*}, A_{0}^{*}\right)$ satisfying the following conditions:

1) $\omega_{G}\left(A^{H}\right)=A^{H}$

2) $\operatorname{res}_{A_{H, 0}}\left(i_{0}^{*-1} \circ \omega_{G} \circ i_{0}^{*}\right)=\omega$;

3) $\omega_{G}\left(R^{+}\left(M^{H}, T_{0}^{*}\right)\right) \subset R^{+}\left(G^{*}, T_{0}^{*}\right)$.

Proof. Let $\omega^{\prime}=i_{0}^{*} \circ \omega \circ i_{0}^{*-1}$, then we have $\omega^{\prime} \in \Omega\left(G^{*}, T_{0}^{*}\right)$. Put $A_{1}=A_{2}=A^{H}$ and $g=n_{\omega^{\prime}}$. Then apply Lemma 4.2.

We define a homomorphism

$$
i^{*}: \Omega\left(H, A_{H, 0}\right) \longrightarrow \Omega\left(G^{*}, A_{0}^{*}\right)
$$

by putting $i^{*}(\omega)=\omega_{G}$.

Let $R\left(G^{*}, A^{H}\right)_{\text {sym }}$ be the subset of $\operatorname{res}_{H}\left(R\left(G^{*}, A_{0}^{*}\right)\right)-\{0\}$ consisting of the $R(G)$-symmetric roots on $A^{H}$. By applying Lemma 4.3 to the reflection $s_{\alpha}$ of $\alpha \in R\left(H, A_{H, 0}\right)$, we have the following corollary.

Corollary 4.4. We have

$$
i_{0}^{*}\left(R\left(H, A_{H, 0}\right)\right) \subset R\left(G^{*}, A^{H}\right)_{\mathrm{sym}} .
$$

For the sake of brevity, we assume that $\mathcal{H}={ }^{L} H$ until the end of this section. We denote by $H_{G \text {-reg }}$ the set of strongly $G$-regular elements in $H(F)$. For $\gamma^{H} \in H_{G \text {-reg }}$ and $\gamma^{G} \in G_{\text {reg }}$, let $\Delta_{G, H}\left(\gamma^{H}, \gamma^{G}\right)$ be the Langlands-Shelstad transfer factor. Since we normalized the orbital integral, we define $\Delta_{G, H}$ by the product of $\Delta_{\mathrm{I}}, \Delta_{\mathrm{II}}$ and $\Delta_{\mathrm{III}}$ in this paper. Because we have to define the transfer of virtual characters, we assume the fundamental lemma for groups [Art96, Hypothesis 3.1] and for Lie algebras [Wa197, Conjecture 1.3] in this paper. Then [Wal97, Corollary 1.7] asserts that for each $f^{G} \in C_{c}^{\infty}(G)$, there exists $f^{H} \in C_{c}^{\infty}(H)$ such that

$$
I^{\mathrm{st}}\left(\gamma^{H}, f^{H}\right)=\sum_{\gamma \in \Gamma(G)} \Delta_{G, H}\left(\gamma^{H}, \gamma\right) I\left(\gamma, f^{G}\right),
$$

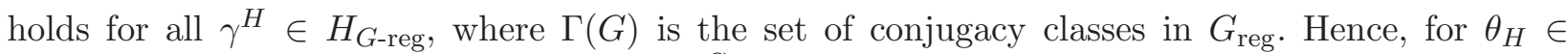
$\mathbb{C}[\Pi(H)]^{\text {st }}$, we can define a linear form $\operatorname{Tran}_{H}^{G}\left(\theta_{H}\right)$ on $C_{c}^{\infty}(G)$ by the relation

$$
\operatorname{Tran}_{H}^{G}\left(\theta_{H}\right)\left(f^{G}\right)=\theta_{H}\left(f^{H}\right) .
$$

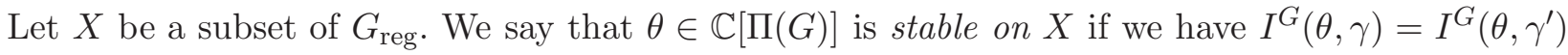
for all $\gamma, \gamma^{\prime} \in X$ that are stably conjugate.

Lemma 4.5. Let $L$ be a standard Levi subgroup of $H$. If $\theta_{L} \in \mathbb{C}[\Pi(L)]$ is invariant under the adjoint action of $\operatorname{Norm}(L, H(F))$ and if $i_{L}^{H}\left(\theta_{L}\right)$ is stable on $L_{\mathrm{ell}} \cap H_{\mathrm{reg}}$, then $\theta_{L}$ is stable on $L_{\mathrm{ell}}$.

Proof. Let $\gamma \in L_{\mathrm{ell}} \cap H_{\mathrm{reg}}$ and $T=\operatorname{Cent}(\gamma, H)$. Let $T^{\prime}$ be a maximal torus of $L$ such that $I_{\text {conj }}^{H, L}\left(T^{\prime}, T\right) \neq \emptyset$. Let $i \in I_{\text {conj }}^{H, L}\left(T^{\prime}, T\right)$, then there exists $h \in H(F)$ such that $i=\operatorname{Int} h$. Since $\gamma \in$ $L_{\text {ell }} \cap H_{\text {reg }}$, we have $L=\operatorname{Cent}\left(A_{T}, H\right)$. Therefore, $L$ is determined by $\gamma$. Since $i^{-1}(\gamma) \in L_{\text {ell }}, L$ is 


\section{ON FUNCTORIALITY OF ZELEVINSKI INVOLUTIONS}

also determined by $i^{-1}(\gamma)$. Hence, we have $\operatorname{Int} h(L)=L$. Thus $h \in \operatorname{Norm}(L, H(F))$. Therefore, by (3.1), we have

$$
I^{H}\left(i_{L}^{H}\left(\theta_{L}\right), \gamma\right)=\sum_{h \in \operatorname{Norm}(L, H(F)) / L(F)} I^{L}\left(\theta_{L}, \operatorname{Int} h^{-1}(\gamma)\right) .
$$

Since $\theta_{L}$ is $\operatorname{Norm}(L, H(F))$-invariant, this shows that

$$
I^{H}\left(i_{L}^{H}\left(\theta_{L}\right), \gamma\right)=n \cdot I^{L}\left(\theta_{L}, \gamma\right),
$$

where $n=\sharp \operatorname{Norm}(L, H(F)) / L(F)$. Since $i_{L}^{H}\left(\theta_{L}\right)$ is stable on $L_{\text {ell }} \cap H_{\text {reg }}$, this implies that $\theta_{L}$ is stable on $L_{\text {ell }}$.

Proposition 4.6. Assume the fundamental lemma for groups and for Lie algebras. Then for $\theta_{H} \in$ $\mathbb{C}[\Pi(H)]^{\text {st }}$, we have

$$
\operatorname{Tran}_{H}^{G}\left(\theta_{H}\right) \in \mathbb{C}[\Pi(G)]
$$

Proof. For $L \in \mathcal{L}^{H}$, we write $\tilde{T}_{\text {ell }}(L)$ for the set of orbits of (essential) elliptic triplets as in [Art96, p. 530]. We also define $\tilde{T}_{\text {ell }}(L)_{\mathbb{C}}$ as in $\left[\operatorname{Art96}\right.$, p. 530]. We denote by $\mathbb{C}\left[\tilde{T}_{\text {ell }}(L)\right]$ the subspace of $\mathbb{C}[\Pi(L)]$ generated by the virtual characters attached to $\tilde{T}_{\text {ell }}(L)$ and by $\mathbb{C}\left[\tilde{T}_{\text {ell }}(L)_{\mathbb{C}}\right]$ the subspace of $\mathbb{C}[\Pi(L)]$ generated by the virtual characters attached to $\tilde{T}_{\text {ell }}(L)_{\mathbb{C}}$. Choose a subset $\tilde{\mathcal{L}^{H}}$ of $\mathcal{L}^{H}$ so that each standard Levi subgroup of $H$ has one and only one associate standard Levi subgroup in $\tilde{\mathcal{L}}^{H}$. Since $\theta_{H}$ is a virtual character, we can write $\theta_{H}$ as $\theta_{H}=\sum_{L \in \tilde{\mathcal{L}}^{H}} i_{L}^{H}\left(\sigma_{L}\right)$ with $\sigma_{L} \in \mathbb{C}\left[\tilde{T}_{\text {ell }}(L)_{\mathbb{C}}\right]$. Moreover, we may assume that $\sigma_{L}$ is invariant under the action of $\operatorname{Norm}(L, H(F))$. We claim that $\sigma_{L} \in \mathbb{C}[\Pi(L)]^{\text {st }}$. We prove this by induction on $r(H)-r(L)$. Let $L \in \tilde{\mathcal{L}}^{H}$. Then by the inductive assumption,

$$
\theta_{H}-\sum_{\substack{L^{\prime} \in \tilde{\mathcal{L}^{H}} \\ r\left(L^{\prime}\right)>r(L)}} i_{L^{\prime}}^{H}\left(\sigma_{L^{\prime}}\right)=\sum_{\substack{L^{\prime} \in \tilde{\mathcal{L}^{H}} \\ r\left(L^{\prime}\right) \leqslant r(L)}} i_{L^{\prime}}^{H}\left(\sigma_{L^{\prime}}\right)
$$

is a stable virtual character. We see that $i_{L}^{H}\left(\sigma_{L}\right)$ is the only term which is not zero on $L_{\text {ell }} \cap H_{\text {reg }}$ in the right-hand side. Thus $i_{L}^{H}\left(\sigma_{L}\right)$ is stable on $L_{\text {ell }} \cap H_{\text {reg. }}$. Therefore, Lemma 4.5 asserts that $\sigma_{L}$ is stable on $L_{\text {ell }}$. Since $\sigma_{L}$ is a finite linear combination of virtual characters attached to $\tilde{T}_{\text {ell }}(L)_{\mathbb{C}}$, [Art96, Theorem 6.1] implies that $\sigma_{L}$ is a stable virtual character. We have thus proved the claim. So, it is enough to show that $\operatorname{Tran}_{H}^{G} \circ i_{L}^{H}\left(\sigma_{L}\right) \in \mathbb{C}[\Pi(G)]$. Put $\mathbb{C}\left[\tilde{T}_{\text {ell }}(L)\right]^{\text {st }}=\mathbb{C}\left[\tilde{T}_{\text {ell }}(L)\right] \cap \mathbb{C}[\Pi(L)]^{\text {st }}$. It is not difficult to see that if $\operatorname{Tran}_{H}^{G} \circ i_{L}^{H}$ is not zero, then there exist a standard Levi subgroup $M$ of $G$, a set of elliptic endoscopic data $\left(L,{ }^{L} L, s_{L}, \xi_{L}\right)$ for $M$ and a non-zero constant $c$ such that

$$
\operatorname{Tran}_{H}^{G} \circ i_{L}^{H}=c \cdot i_{M}^{G} \circ \operatorname{Tran}_{L}^{M} .
$$

(Note that the transfer factor is defined up to a constant factor.) By applying [Art96, Theorem 6.2] to the set of endoscopic data $\left(L,{ }^{L} L, s_{L}, \xi_{L}\right)$, we can show that

$$
\operatorname{Tran}_{H}^{G} \circ i_{L}^{H}\left(\mathbb{C}\left[\tilde{T}_{\text {ell }}(L)\right]^{\text {st }}\right) \subset \mathbb{C}[\Pi(G)] .
$$

(By using [Art96, Lemma 5.2], we can show that the linear form $f \longrightarrow f_{g r}^{\prime}\left(\phi^{\prime}\right)$ in [Art96, Theorem 6.2] is a virtual character.) It is easy to extend this to $\mathbb{C}\left[\tilde{T}_{\text {ell }}(L)_{\mathbb{C}}\right]^{\text {st }}=\mathbb{C}\left[\tilde{T}_{\text {ell }}(L)_{\mathbb{C}}\right] \cap \mathbb{C}[\Pi(L)]^{\text {st }}$.

Let $T^{H}$ be a maximal torus of $H$ and $T^{G}$ a maximal torus of $G$. We say that an isomorphism $i: T^{H} \longrightarrow T^{G}$ is admissible if $i$ is defined over $F$ and if there exist $h \in H(\bar{F})$ and $g \in G(\bar{F})$ such that $\operatorname{Int} g\left(T_{0}\right)=T^{G}$, Int $h\left(T_{H, 0}\right)=T^{H}$ and

$$
i=\operatorname{Int} g \circ \varphi^{-1} \circ i_{0}^{*} \circ \operatorname{Int} h^{-1} .
$$

We denote by $I^{G, H}\left(T^{H}, T^{G}\right)$ the set of admissible isomorphisms from $T^{H}$ to $T^{G}$. Then $\Omega\left(H, T^{H}\right)_{F}$ and $\Omega\left(G, T^{G}\right)_{F}$ act on $I^{G, H}\left(T^{H}, T^{G}\right)$ from the right and left, respectively. Let $\gamma^{H} \in T^{H}(F)$ be a 


\section{K. HiRAGA}

strongly $G$-regular element and $\gamma^{G} \in T^{G}(F)$ a strongly regular element. Then we say that $\gamma^{H}$ is an image (or a $(G, H)$-image) of $\gamma^{G}$ if there exists an admissible isomorphism $i \in I^{G, H}\left(T^{H}, T^{G}\right.$ ) such that $i\left(\gamma^{H}\right)=\gamma^{G}$. In this paper, we also say that $T^{H}$ is an image of $T^{G}$ if there exists an admissible isomorphism $T^{H} \longrightarrow T^{G}$. Let $\mathcal{T}^{H}$ be a system of representatives for the stable conjugacy classes of maximal tori of $H$. Since $\operatorname{Tran}_{H}^{G}\left(\theta_{H}\right)$ is a virtual character, we can consider a function $I^{G}\left(\operatorname{Tran}_{H}^{G}\left(\theta_{H}\right)\right)$ on $G_{\text {reg }}$. By a routine calculation, we can prove the following formula from the definition of $\operatorname{Tran}_{H}^{G}$.

Lemma 4.7. For $\gamma \in G_{\text {reg }}$ and $\theta_{H} \in \mathbb{C}[\Pi(H)]^{\text {st }}$, we have

$$
I^{G}\left(\operatorname{Tran}_{H}^{G}\left(\theta_{H}\right), \gamma\right)=\sum_{T^{H} \in \mathcal{T}^{H}} \sum_{i \in I^{G, H}\left(T^{H}, G_{\gamma}\right) / \Omega\left(H, T^{H}\right)_{F}} \Delta_{G, H}\left(i^{-1}(\gamma), \gamma\right) I^{H}\left(\theta_{H}, i^{-1}(\gamma)\right) .
$$

\section{Analogue of the formula of Bernstein-Zelevinski}

In this section, we fix $M \in \mathcal{L}^{G}$. We put $M^{*}=\varphi(M)$. By Corollary 4.4 , we have $i_{0}^{*}\left(R\left(H, A_{H, 0}\right)\right) \subset$ $R\left(G^{*}, A^{H}\right)_{\text {sym }}$. Therefore, we can define

$$
D_{M^{*}, H} \subset \Omega\left(G^{*}, A_{0}^{*}\right)
$$

as in $\S 2$. We put $D_{M, H}=D_{M^{*}, H}$. Recall that we defined a homomorphism

$$
i^{*}: \Omega\left(H, A_{H, 0}\right) \longrightarrow \Omega\left(G^{*}, A_{0}^{*}\right)
$$

by putting $i^{*}(\omega)=\omega_{G}$, where $\omega_{G}$ is the element in Lemma 4.3. Thus $i^{*}\left(\Omega\left(H, A_{H, 0}\right)\right)$ is the subgroup $\Omega(H)$ in $\S 2$ corresponding to the root system $R(H)=i_{0}^{*}\left(R\left(H, A_{H, 0}\right)\right)$. Therefore, Proposition 2.2 asserts that $D_{M, H}$ is a system of representatives for

$$
\Omega\left(M^{*}, A_{0}^{*}\right) \backslash \Omega\left(G^{*}, A_{0}^{*}\right)_{M, H} / i^{*}\left(\Omega\left(H, A_{H, 0}\right)\right),
$$

where

$$
\Omega\left(G^{*}, A_{0}^{*}\right)_{M, H}=\left\{\omega \in \Omega\left(G^{*}, A_{0}^{*}\right) \mid \omega \circ i_{0}^{*}\left(A_{H, 0}\right) \supset A_{M^{*}}\right\} .
$$

For $\omega \in D_{M, H}$, we put

$$
M_{\omega}=\operatorname{Cent}\left(\left(\omega \circ i_{0}^{*}\right)^{-1}\left(A_{M^{*}}\right), H\right) .
$$

Then it is easy to see that

$$
i_{0}^{*}\left(R\left(M_{\omega}, A_{H, 0}\right)\right)=i_{0}^{*}\left(R\left(H, A_{H, 0}\right)\right) \cap \operatorname{res}_{H} \omega^{-1}\left(R\left(M^{*}, A_{0}^{*}\right)\right) .
$$

Thus Lemma 2.3 asserts that $M_{\omega}$ is a standard Levi subgroup of $H$. It is also easy to see that

$$
\Omega\left(M_{\omega}, T_{H, 0}\right)=\Omega\left(H, T_{H, 0}\right) \cap\left(\omega \circ i_{0}^{*}\right)^{-1} \circ \Omega\left(M^{*}, T_{0}^{*}\right) \circ\left(\omega \circ i_{0}^{*}\right) .
$$

Let $\hat{M}_{\omega}$ be the dual group of $M_{\omega}$. Since $M_{\omega}$ is a standard Levi subgroup, we can regard $\hat{M}_{\omega}$ as a standard Levi subgroup of $\hat{H}$. Let $\hat{n}_{\omega} \in \operatorname{Norm}(\mathcal{T}, \hat{G})$ be a representative for $\omega \in \Omega\left(G^{*}, T_{0}^{*}\right)=$ $\Omega(\hat{G}, \mathcal{T})$. We put

$$
\begin{aligned}
\xi_{\omega} & =\operatorname{Int} \hat{n}_{\omega} \circ \xi, \\
s_{\omega}^{\prime} & =\operatorname{Int} \hat{n}_{\omega}(s) .
\end{aligned}
$$

By using $\omega \circ i_{0}^{*}\left(R\left(M_{\omega}, T_{H, 0}\right)\right)=\omega \circ i_{0}^{*}\left(R\left(H, T_{H, 0}\right)\right) \cap R\left(M^{*}, T_{0}^{*}\right)$, we have $\xi_{\omega}\left(\hat{M}_{\omega}\right)=\operatorname{Cent}\left(s_{\omega}^{\prime}, \hat{M}\right)^{0}$, where ${ }^{0}$ denotes the identity component. We can choose $a_{\omega} \in\left(Z_{\hat{M}}^{\Gamma}\right)^{0}$ such that $\operatorname{Cent}\left(a_{\omega} s_{\omega}^{\prime}, \hat{G}\right)^{0}=\hat{M}_{\omega}$. We put $s_{\omega}=a_{\omega} s_{\omega}^{\prime}$. Let $c: W_{F} \longrightarrow \mathcal{H}$ be a continuous splitting of

$$
1 \longrightarrow \hat{H} \longrightarrow \mathcal{H} \longrightarrow W_{F} \longrightarrow 1 \text {. }
$$

By the proof of $\left[\mathrm{KS} 99\right.$, Lemma 2.2.A], we may assume that $\operatorname{Int} c\left(W_{F}\right)$ preserves the pair $\left(\mathcal{B}_{H}, \mathcal{T}_{H}\right)$. We put $\mathcal{M}_{\omega}=\hat{M}_{\omega} \cdot c\left(W_{F}\right)$. Then $\mathcal{M}_{\omega}$ is a subgroup of $\mathcal{H}$. 


\section{ON FUNCTORIALITY OF ZELEVINSKI INVOLUTIONS}

Lemma 5.1. The sets $\left(M_{\omega}, \mathcal{M}_{\omega}, s_{\omega}^{\prime}, \xi_{\omega}\right)$ and $\left(M_{\omega}, \mathcal{M}_{\omega}, s_{\omega}, \xi_{\omega}\right)$ are equivalent endoscopic data for $M$, and $\left(M_{\omega}, \mathcal{M}_{\omega}, s_{\omega}, \xi_{\omega}\right)$ is a set of endoscopic data for $G$.

Proof. Let $w \in W_{F}$ and let $\sigma \in \Gamma$ be the image of $w$. We denote by $\sigma_{H}$ the action of $\sigma$ on $T_{H, 0}$ and by $\sigma_{G^{*}}$ the action of $\sigma$ on $T_{0}^{*}$. Then there exists $\omega^{\prime} \in \Omega\left(G^{*}, T_{0}^{*}\right)$ such that $i_{0}^{*} \circ \sigma_{H} \circ i_{0}^{*-1}=\omega^{\prime} \circ \sigma_{G^{*}}$. Put $\omega^{\prime \prime}=\omega \omega^{\prime} \omega^{-1}$. Then we have $\left(\omega \circ i_{0}^{*}\right) \circ \sigma_{H} \circ\left(\omega \circ i_{0}^{*}\right)^{-1}=\omega^{\prime \prime} \circ \sigma_{G^{*}}$. By $\omega \in D_{M, H}$, we have $\omega \circ i_{0}^{*}\left(A_{H, 0}\right) \supset A_{M^{*}}$. Since $A_{H, 0}$ and $A_{0}^{*}$ are split tori, this shows that the action of $\omega^{\prime \prime}$ on $A_{M^{*}}$ is trivial. Thus $\omega^{\prime \prime} \in \Omega\left(M^{*}, T_{0}^{*}\right)$. Since $\operatorname{Int} c(w)$ preserves the pair $\left(\mathcal{B}_{H}, \mathcal{T}_{H}\right)$, the action of Int $c(w)$ on $\mathcal{T}_{H}$ is equal to the action of $\sigma_{H}$ on $\mathcal{T}_{H} \cong \hat{T}_{H, 0}$. Therefore, we have

$$
\xi_{\omega}(c(w)) \in \operatorname{Norm}(\mathcal{T}, \hat{M}) \rtimes \sigma \subset^{L} M .
$$

Hence, $\xi_{\omega}\left(\mathcal{M}_{\omega}\right) \subset{ }^{L} M$. The other parts of the proof are easily verified.

It is easy to see that the equivalence classes of the endoscopic data do not depend on the choice of $\hat{n}_{\omega}$ and $c$. We put $\mathcal{B}_{M_{\omega}}=\mathcal{B}_{H} \cap \hat{M}_{\omega}$. Then the restriction $\left(\mathcal{B}_{M_{\omega}}, \mathcal{T}_{H},\left\{\mathcal{Y}_{\check{\alpha}}\right\}\right)$ of the $\Gamma$-splitting of $H$ is a $\Gamma$-splitting of $\hat{M}_{\omega}$. We have $s_{\omega} \in \mathcal{T}$ and $\xi_{\omega}\left(\mathcal{T}_{H}\right)=\mathcal{T}$. Moreover, since $\omega \in D_{M, H}$, we have $\omega \circ i_{0}^{*}\left(R^{+}\left(H, T_{H, 0}\right)\right) \subset R^{+}\left(G^{*}, T_{0}^{*}\right)$. This implies that $\xi_{\omega}\left(\mathcal{B}_{M_{\omega}}\right) \subset \mathcal{B}$. Let $\left(H_{1}, \xi_{H_{1}}\right)$ be a $z$-pair (see $[K S 99, \S 2.2])$. Let $\left(\mathcal{B}_{H_{1}}, \mathcal{T}_{H_{1}},\left\{\mathcal{Y}_{\check{\alpha}}\right\}\right)$ be the $\Gamma$-splitting of $\hat{H}_{1}$ obtained from the $\Gamma$-splitting $\left(\mathcal{B}_{H}, \mathcal{T}_{H},\left\{\mathcal{Y}_{\check{\alpha}}\right\}\right)$. Then $\xi_{H_{1}} \circ c\left(W_{F}\right)$ preserves the pair $\left(\mathcal{B}_{H_{1}}, \mathcal{T}_{H_{1}}\right)$. Let $M_{\omega, 1}$ be the standard Levi subgroup of $H_{1}$ corresponding to $M_{\omega}$. Then the restriction of the $L$-group data for $H_{1}$ defines an $L$-group data for $M_{\omega, 1}$. Let ${ }^{L} M_{\omega, 1} \hookrightarrow{ }^{L} H_{1}$ be the natural embedding. Then it is easy to see that $\xi_{H_{1}}\left(\mathcal{M}_{\omega}\right)$ is contained in ${ }^{L} M_{\omega, 1} \subset{ }^{L} H_{1}$. Therefore, $\left(M_{\omega, 1}, \xi_{H_{1}}\right)$ is a $z$-pair for $\mathcal{M}_{\omega}$.

For the sake of brevity, we assume that $\mathcal{H}={ }^{L} H$ until the end of $\S 6$. Then $\mathcal{M}_{\omega}$ is the image of the natural embedding ${ }^{L} M_{\omega} \hookrightarrow{ }^{L} H$. We identify $\mathcal{M}_{\omega}$ with ${ }^{L} M_{\omega}$. Let $\left(M^{*} \cap B_{0}^{*}, T_{0}^{*},\left\{X_{\alpha}\right\}\right)$ be the restriction of the $F$-splitting $\left(B_{0}^{*}, T_{0}^{*},\left\{X_{\alpha}\right\}\right)$ to $M^{*}$ and let $\left(M_{\omega} \cap B_{H, 0}, T_{H, 0},\left\{Y_{\alpha}\right\}\right)$ be the restriction of the $F$-splitting $\left(B_{H, 0}, T_{H, 0},\left\{Y_{\alpha}\right\}\right)$ to $M_{\omega}$. We write $i_{0}^{M_{\omega}}$ for $\omega \circ i_{0}^{*}$. Then $i_{0}^{M_{\omega}}$ is the dual of $\xi_{\omega}^{-1}$. We put

$$
\Gamma\left(M_{\omega}, M\right)=\left\{\left(\gamma^{M_{\omega}}, \gamma^{M}\right) \in M_{\omega, G \text {-reg }} \times M_{G \text {-reg }} \mid \gamma^{M_{\omega}} \text { is an }\left(M, M_{\omega}\right) \text {-image of } \gamma^{M}\right\} .
$$

We denote by $\Delta_{M, M_{\omega}}$ the Langlands-Shelstad transfer factor of $M_{\omega}$. Note that $\Delta_{M, M_{\omega}}$ is defined up to a constant factor.

Lemma 5.2. There exists $c \in \mathbb{C}^{\times}$such that

$$
\Delta_{G, H}\left(\gamma^{M_{\omega}}, \gamma^{M}\right)=c \cdot \Delta_{M, M_{\omega}}\left(\gamma^{M_{\omega}}, \gamma^{M}\right)
$$

for all $\left(\gamma^{M_{\omega}}, \gamma^{M}\right) \in \Gamma\left(M_{\omega}, M\right)$.

Proof. We write $\Delta_{G, H}^{\prime}$ for the Langlands-Shelstad transfer factor of the set of endoscopic data $\left(H,{ }^{L} H, s_{\omega}^{\prime}, \xi_{\omega}\right)$. Since the relative transfer factor of $\left(H,{ }^{L} H, s_{\omega}^{\prime}, \xi_{\omega}\right)$ is equal to the relative transfer factor of $\left(H,{ }^{L} H, s, \xi\right)$, it is enough to show that

$$
\Delta_{G, H}^{\prime}\left(\gamma^{M_{\omega}}, \gamma^{M} ; \bar{\gamma}^{M_{\omega}}, \bar{\gamma}^{M}\right)=\Delta_{M, M_{\omega}}\left(\gamma^{M_{\omega}}, \gamma^{M} ; \bar{\gamma}^{M_{\omega}}, \bar{\gamma}^{M}\right)
$$

for all $\left(\gamma^{M_{\omega}}, \gamma^{M}\right),\left(\bar{\gamma}^{M_{\omega}}, \bar{\gamma}^{M}\right) \in \Gamma\left(M_{\omega}, M\right)$. We remark that $\xi_{\omega}\left(\mathcal{B}_{H}\right) \subset \mathcal{B}$. Put $T^{M_{\omega}}=\operatorname{Cent}\left(\gamma^{M_{\omega}}, M_{\omega}\right)$ and $T^{M}=\operatorname{Cent}\left(\gamma^{M}, M\right)$. Fix a maximal torus $T^{*} \subset M^{*}$ such that $T^{M_{\omega}}$ is an image of $T^{*}$ and fix $i \in I^{M^{*}, M_{\omega}}\left(T^{M_{\omega}}, T^{*}\right)$. We regard $i$ as an admissible embedding of $T^{M_{\omega}} \subset H$ into $G^{*}$. Choose $h \in M_{\omega}(\bar{F})$ and $m \in M^{*}(\bar{F})$ such that $\operatorname{Int} h\left(B_{H, 0}, T_{H, 0}\right)$ and $\operatorname{Int} m\left(B_{0}^{*}, T_{0}^{*}\right)$ determine $i$. Then $\operatorname{Int} h\left(M_{\omega} \cap B_{H, 0}, T_{H, 0}\right)$ and $\operatorname{Int} m\left(M^{*} \cap B_{0}^{*}, T_{0}^{*}\right)$ also determine $i$. Choose $a$-data $\left\{a_{\alpha}\right\}$ for $R\left(G^{*}, T^{*}\right)$. Define $a$-data for $R\left(M^{*}, T^{*}\right)$ by the restriction of $\left\{a_{\alpha}\right\}$ to $R\left(M^{*}, T^{*}\right)$. Let $\left\{\chi_{\alpha}\right\}$ be $\chi$-data for $R\left(G^{*}, T^{*}\right)$. If $\alpha \in R\left(G^{*}, T^{*}\right)-R\left(M^{*}, T^{*}\right)$, then $\alpha$ is asymmetric. Therefore, we can choose $\left\{\chi_{\alpha}\right\}$ such that $\chi_{\alpha} \equiv 1$ for all $\alpha \in R\left(G^{*}, T^{*}\right)-R\left(M^{*}, T^{*}\right)$ (see [Art96, p. 521]). Define $\chi$-data for $R\left(M^{*}, T^{*}\right)$ by the restriction of $\left\{\chi_{\alpha}\right\}$ to $R\left(M^{*}, T^{*}\right)$. We also fix data for $\bar{\gamma}^{M_{\omega}}$ and $\bar{\gamma}^{M}$ similarly. 


\section{K. HiRAGA}

Now, by using a similar argument to the proof of [Wal97, Lemma 6.5], we can show the required identities for $\Delta_{\mathrm{I}}, \Delta_{\mathbb{I}}$ and $\Delta_{\mathbb{I I}_{1}}$. Therefore, it remains to show the identity for $\Delta_{\mathbb{I I}_{2}}$. Define $\mathbf{a}^{G} \in$ $H^{1}\left(W_{F}, \hat{T}^{*}\right)$ for $\left(H,{ }^{L} H, s_{\omega}^{\prime}, \xi_{\omega}\right)$ and $\mathbf{a}^{M} \in H^{1}\left(W_{F}, \hat{T}^{*}\right)$ for $\left(M_{\omega},{ }^{L} M_{\omega}, s_{\omega}^{\prime}, \xi_{\omega}\right)$ as in [LS87, $\left.\S 3.5\right]$. By the choice of splittings, $\chi$-data, pairs and $i$, we can easily show that $\mathbf{a}^{G}=\mathbf{a}^{M}$. Let $\Delta_{\mathrm{II}_{2}}^{G}$ be the $\Delta_{\mathbb{I}_{2}}$ factor of $\left(H,{ }^{L} H, s_{\omega}^{\prime}, \xi_{\omega}\right)$ and $\Delta_{\mathbb{I I}_{2}}^{M}$ the $\Delta_{\mathbb{I}_{2}}$ factor of $\left(M_{\omega},{ }^{L} M_{\omega}, s_{\omega}^{\prime}, \xi_{\omega}\right)$, then the above identity implies that $\Delta_{\mathrm{II}_{2}}^{G}\left(\gamma^{M_{\omega}}, \gamma^{M}\right)=\Delta_{\mathrm{II}_{2}}^{M}\left(\gamma^{M_{\omega}}, \gamma^{M}\right)$. This completes the proof.

We replace $\Delta_{M, M_{\omega}}$ by $c \cdot \Delta_{M, M_{\omega}}$. Then we have

$$
\Delta_{G, H}\left(\gamma^{M_{\omega}}, \gamma^{M}\right)=\Delta_{M, M_{\omega}}\left(\gamma^{M_{\omega}}, \gamma^{M}\right)
$$

for all $\left(\gamma^{M_{\omega}}, \gamma^{M}\right) \in \Gamma\left(M_{\omega}, M\right)$.

Fix a maximal torus $T^{G}$ of $M$. Assume that $A_{T^{G}}$ is a standard subtorus of $A_{0}$. Let $\left\{T_{1}^{H}, \ldots, T_{r}^{H}\right\}$ be a system of representatives for the stable conjugacy classes of maximal tori of $H$ that are images of $T^{G}$. We may assume that $A_{T_{1}^{H}}, \ldots, A_{T_{r}^{H}}$ are standard subtori of $A_{H, 0}$. For $i=1, \ldots, r$ and $\omega \in D_{M, H}$, let $\left\{T_{i 1}^{\omega}, \ldots, T_{i r_{\omega, i}}^{\omega}\right\}$ be a system of representatives for the stable conjugacy classes of maximal tori of $M_{\omega}$ that are stably $H$-conjugate to $T_{i}^{H}$. For each $T_{i j}^{\omega}$, fix $z_{i j}^{\omega} \in H(\bar{F})$ such that Int $z_{i j}^{\omega}\left(T_{i}^{H}\right)=T_{i j}^{\omega}$ and that

$$
\operatorname{Int} z_{i j}^{\omega}: T_{i}^{H} \longrightarrow T_{i j}^{\omega}
$$

is defined over $F$. We put

$$
Y=\bigcup_{i} I^{H, G}\left(T_{i}^{H}, T^{G}\right) / \Omega\left(H, T_{i}^{H}\right)_{F}
$$

Then we may regard $Y$ as the set

$$
\left\{\left(T_{i}^{H}, \tilde{\imath}^{H}\right) \mid 1 \leqslant i \leqslant r, \tilde{\imath}^{H} \in I^{H, G}\left(T_{i}^{H}, T^{G}\right) / \Omega\left(H, T_{i}^{H}\right)_{F}\right\} .
$$

For $\omega \in D_{M, H}$, we put

$$
\tilde{Y}_{\omega}=\bigcup_{i, j} I^{M_{\omega}, M}\left(T_{i j}^{\omega}, T^{G}\right) / \Omega\left(M_{\omega}, T_{i j}^{\omega}\right)_{F}
$$

We also put

$$
\tilde{Y}=\bigcup_{\omega \in D_{M, H}} \tilde{Y}_{\omega} .
$$

Then $\tilde{Y}$ can be regarded as the set of $\left(\omega, T_{i j}^{\omega}, \tilde{\imath}^{\omega}\right)$, where $\omega \in D_{M, H}, 1 \leqslant i \leqslant r, 1 \leqslant j \leqslant r_{\omega, i}$ and $\tilde{\imath}^{\omega} \in I^{M_{\omega}, M}\left(T_{i j}^{\omega}, T^{G}\right) / \Omega\left(M_{\omega}, T_{i j}^{\omega}\right)_{F}$. It is easy to see that $T_{i}^{H}$ is stably $H$-conjugate to $T_{i^{\prime} j}^{\omega}$ if and only if $i=i^{\prime}$. Moreover, if $(i, j) \neq\left(i^{\prime}, j^{\prime}\right)$, then $T_{i j}^{\omega}$ is not stably $M_{\omega}$-conjugate to $T_{i^{\prime} j^{\prime}}^{\omega}$. We say that $\left(T_{i^{\prime}}^{H}, \tilde{\imath}^{H}\right) \in Y$ corresponds to $\left(\omega, T_{i j}^{\omega}, \tilde{\imath}^{\omega}\right) \in \tilde{Y}$ if $i^{\prime}=i$ and if there exist a representative $i^{H}$ for $\tilde{\imath}^{H}$, a representative $i^{\omega}$ for $\tilde{\imath}^{\omega}$ and $\omega_{H} \in \Omega\left(H, T_{i^{\prime}}^{H}\right)_{F}$ such that

$$
i^{H}=i^{\omega} \circ \operatorname{Int} z_{i j}^{\omega} \circ \omega_{H} \text {. }
$$

Proposition 5.3. The above correspondence is a one-to-one correspondence between $Y$ and $\tilde{Y}$.

It is enough to prove the one-to-one correspondence for each $i=1, \ldots, r$. Thus we fix $i \in$ $\{1, \ldots, r\}$. Put $T^{H}=T_{i}^{H}, T_{j}^{\omega}=T_{i j}^{\omega}, z_{j}^{\omega}=z_{i j}^{\omega}$ and $r_{\omega}=r_{\omega, i}$. Put $M_{T^{H}}=\operatorname{Cent}\left(A_{T^{H}}, H\right)$ and $M_{T^{G}}=$ $\operatorname{Cent}\left(A_{T^{G}}, G\right)$. Then, by $T^{G} \subset M$, we have $M_{T^{G}} \subset M$. Since $A_{T^{H}}$ is a standard subtorus of $A_{H, 0}$, we have $T_{H, 0} \subset M_{T^{H}}$. Let $i^{H} \in I^{H, G}\left(T^{H}, T^{G}\right)$, then there exist $g \in G(\bar{F})$ and $h \in M_{T^{H}}(\bar{F}) \subset H(\bar{F})$ such that $\operatorname{Int} h\left(T_{H, 0}\right)=T^{H}$, Int $g\left(T_{0}\right)=T^{G}$ and

$$
i^{H}=\operatorname{Int} g \circ \varphi^{-1} \circ i_{0}^{*} \circ \operatorname{Int} h^{-1},
$$




\section{ON FUNCTORIALITY OF ZELEVINSKI INVOLUTIONS}

since $A_{T^{H}}$ is a standard subtorus of $A_{H, 0}$. Fix $m \in M_{T^{G}}(\bar{F})$ such that $T^{G}=\operatorname{Int} m\left(T_{0}\right)$. Then we have $A_{T^{G}} \subset \operatorname{Int} m\left(A_{0}\right)$.

Lemma 5.4. There exists $\omega^{\prime} \in \Omega\left(G^{*}, A_{0}^{*}\right)$ such that

$$
\left.i^{H}\right|_{A_{T H}}=\left.\operatorname{Int} m \circ \varphi^{-1} \circ \omega^{\prime} \circ i_{0}^{*} \circ \operatorname{Int} h^{-1}\right|_{A_{T H}}
$$

and

$$
\omega^{\prime} \circ i_{0}^{*}\left(A_{H, 0}\right) \supset A_{M^{*}}
$$

Proof. Put

$$
\phi=\varphi \circ \operatorname{Int} m^{-1} \circ i^{H} \circ \operatorname{Int} h \circ i_{0}^{*-1}: T_{0}^{*} \longrightarrow T_{0}^{*} .
$$

Since $\phi \circ i_{0}^{*}\left(A_{T^{H}}\right)=\varphi\left(A_{T^{G}}\right)$, we have $\phi \circ i_{0}^{*}\left(A_{T^{H}}\right) \subset A_{0}^{*}$. Put $A_{1}=i_{0}^{*}\left(A_{T^{H}}\right) \subset A_{0}^{*}$ and $A_{2}=$ $\phi \circ i_{0}^{*}\left(A_{T^{H}}\right) \subset A_{0}^{*}$. Since $\phi=\operatorname{Int}\left(\varphi\left(m^{-1} g\right)\right)$, Lemma 4.2 asserts that there exists $\omega^{\prime} \in \Omega\left(G^{*}, A_{0}^{*}\right)$ such that $\omega^{\prime}\left(A_{1}\right)=A_{2}$ and $\left.\omega^{\prime}\right|_{A_{1}}=\left.\phi\right|_{A_{1}}$. This implies the first relation. Since

$$
A_{M^{*}} \subset \varphi\left(A_{T^{G}}\right)=A_{2}=\phi \circ i_{0}^{*}\left(A_{T^{H}}\right)=\omega^{\prime} \circ i_{0}^{*}\left(A_{T^{H}}\right),
$$

$\omega^{\prime}$ satisfies the second property.

Proposition 2.2 asserts that the intersection of $D_{M, H}$ and $\Omega\left(M^{*}, A_{0}^{*}\right) \cdot \omega^{\prime} \cdot i^{*}\left(\Omega\left(H, A_{H, 0}\right)\right)$ consists of a single element $\omega$. We have $\omega \Omega\left(M^{H}, T_{0}^{*}\right) \omega^{-1} \subset \Omega\left(M^{*}, T_{0}^{*}\right)$. Recall that we have

$$
i^{*}\left(\omega_{H}\right) \circ i_{0}^{*} \circ \omega_{H}^{-1} \circ i_{0}^{*-1} \in \Omega\left(M^{H}, T_{0}^{*}\right)
$$

for any $\omega_{H} \in \Omega\left(H, A_{H, 0}\right)=\Omega\left(H, T_{H, 0}\right)_{F}$.

Lemma 5.5. There exist $\omega_{H} \in \Omega\left(H, T_{H, 0}\right)_{F}$ and $\omega_{M^{*}} \in \Omega\left(M^{*}, T_{0}^{*}\right)$ such that

$$
i^{H}=\operatorname{Int} m \circ \varphi^{-1} \circ \omega_{M^{*}} \cdot \omega \circ i_{0}^{*} \circ \omega_{H} \circ \operatorname{Int} h^{-1} .
$$

Proof. It is easy to see that there exists $g^{\prime} \in \operatorname{Norm}\left(T^{G}, G(\bar{F})\right)$ such that

$$
i^{H} \circ\left(\operatorname{Int} m \circ \varphi^{-1} \circ \omega^{\prime} \circ i_{0}^{*} \circ \operatorname{Int} h^{-1}\right)^{-1}=\operatorname{Int} g^{\prime} .
$$

Since $\left.i^{H}\right|_{A_{T^{H}}}=\left.\left(\operatorname{Int} m \circ \varphi^{-1} \circ \omega^{\prime} \circ i_{0}^{*} \circ \operatorname{Int} h^{-1}\right)\right|_{A_{T^{H}}}$, we have $g^{\prime} \in \operatorname{Cent}\left(A_{T^{G}}, G(\bar{F})\right)$. This implies that

$$
\varphi\left(m^{-1} g^{\prime} m\right) \in \operatorname{Norm}\left(T_{0}^{*}, M^{*}(\bar{F})\right) .
$$

Let $\omega_{M^{*}}^{\prime} \in \Omega\left(M^{*}, T_{0}^{*}\right)$ be the image of $\varphi\left(m^{-1} g^{\prime} m\right)$, then

$$
i^{H}=\operatorname{Int} m \circ \varphi^{-1} \circ \omega_{M^{*}}^{\prime} \cdot \omega^{\prime} \circ i_{0}^{*} \circ \operatorname{Int} h^{-1} .
$$

Now, Proposition 2.2 asserts that there exists $\omega_{H} \in \Omega\left(H, T_{H, 0}\right)_{F}$ such that

$$
\omega_{M^{*}}^{\prime} \cdot \omega^{\prime} \in \Omega\left(M^{*}, A_{0}^{*}\right) \cdot \omega \cdot i^{*}\left(\omega_{H}\right) .
$$

Since $\Omega\left(M^{*}, A_{0}^{*}\right) \cdot \omega \cdot i^{*}\left(\omega_{H}\right)=\Omega\left(M^{*}, T_{0}^{*}\right) \omega \circ i_{0}^{*} \circ \omega_{H} \circ i_{0}^{*-1}$, there exists $\omega_{M^{*}} \in \Omega\left(M^{*}, T_{0}^{*}\right)$ such that

$$
\omega_{M^{*}}^{\prime} \cdot \omega^{\prime}=\omega_{M^{*}} \cdot \omega \circ i_{0}^{*} \circ \omega_{H} \circ i_{0}^{*-1} \text {. }
$$

Put

$$
\phi=\omega_{H} \circ \operatorname{Int} h^{-1} \circ i^{H^{-1}}=i_{0}^{*-1} \circ \omega^{-1} \omega_{M^{*}}^{-1} \circ \varphi \circ \operatorname{Int} m^{-1} .
$$

Then $\phi$ is a homomorphism from $T^{G}$ to $T_{H, 0}$. By $\phi=\omega_{H} \circ \operatorname{Int} h^{-1} \circ i^{H^{-1}}$, we have

$$
\sigma(\phi) \circ \phi^{-1}=\omega_{H} \circ \operatorname{Int} \sigma(h)^{-1} h \circ \omega_{H}^{-1}
$$




\section{K. HiRAGA}

for any $\sigma \in \Gamma$. Thus

$$
\sigma(\phi) \circ \phi^{-1} \in \Omega\left(H, T_{H, 0}\right) .
$$

On the other hand, by using $\phi=i_{0}^{*-1} \circ \omega^{-1} \omega_{M^{*}}^{-1} \circ \varphi$ Int $m^{-1}$, we have $\phi\left(A_{M}\right)=i_{0}^{*-1} \circ \omega^{-1}\left(A_{M^{*}}\right) \subset$ $A_{H, 0}$. Since $A_{M}$ and $A_{H, 0}$ are split tori, the restriction of $\sigma(\phi) \circ \phi^{-1}$ to $i_{0}^{*-1} \circ \omega^{-1}\left(A_{M^{*}}\right)$ is identity. Thus

Consequently, we have

$$
\sigma(\phi) \circ \phi^{-1} \in\left(\omega \circ i_{0}^{*}\right)^{-1} \circ \Omega\left(M^{*}, T_{0}^{*}\right) \circ\left(\omega \circ i_{0}^{*}\right) .
$$

$$
\sigma(\phi) \circ \phi^{-1} \in \Omega\left(M_{\omega}, T_{H, 0}\right) .
$$

Therefore [Kot82, Corollary 2.2] asserts that there exists $z \in M_{\omega}(\bar{F})$ such that the homomorphism Int $z \circ \phi$ from $T^{G}$ to $\operatorname{Int} z\left(T_{H, 0}\right)$ is defined over $F$. Since this implies that $T_{H}$ is stably $H$-conjugate to Int $z\left(T_{H, 0}\right)$, we may assume that $\operatorname{Int} z\left(T_{H, 0}\right)$ is equal to $T_{j}^{\omega} \in\left\{T_{1}^{\omega}, \ldots, T_{r_{\omega}}^{\omega}\right\}$. Now, put

$$
i^{\omega}=\phi^{-1} \circ \operatorname{Int} z^{-1} \text {. }
$$

Then we have

$$
i^{\omega}=\operatorname{Int} m \circ \varphi^{-1} \circ \omega_{M^{*}} \omega \circ i_{0}^{*} \circ \operatorname{Int} z^{-1}=\operatorname{Int} m \circ \varphi^{-1}\left(\omega_{M^{*}}\right) \circ \varphi^{-1} \circ i_{0}^{M_{\omega}} \circ \operatorname{Int} z^{-1} .
$$

This implies that $i^{\omega} \in I^{M_{\omega}, M}\left(T_{j}^{\omega}, T^{G}\right)$. Since

$$
i^{\omega-1} \circ i^{H}=\operatorname{Int} z \circ \omega_{H} \circ \operatorname{Int} h^{-1} \in \operatorname{Int} z_{j}^{\omega} \circ \Omega\left(H, T^{H}\right)_{F},
$$

we conclude that $\left(T^{H}, \tilde{\imath}^{H}\right) \in Y$ corresponds to $\left(\omega, T_{j}^{\omega}, \tilde{\imath}^{\omega}\right) \in \tilde{Y}_{\omega}$. Conversely, let $\left(\omega, T_{j}^{\omega}, \tilde{\imath}^{\omega}\right)$ be an element of $\tilde{Y}$ and $i^{\omega}$ a representative for $\tilde{\imath}^{\omega}$. If we put $i^{H}=i^{\omega} \circ \operatorname{Int} z_{j}^{\omega}$, then $\left(T^{H}, \tilde{\imath}^{H}\right) \in Y$ corresponds to $\left(\omega, T_{j}^{\omega}, \tilde{\imath}^{\omega}\right)$.

So, it remains to show that for each element in $Y$, there exists only one element in $\tilde{Y}$ that corresponds to it and vice-versa. Suppose that $\left(T^{H}, \tilde{\imath}^{H}\right) \in Y$ and $\left(T^{H}, \tilde{\imath}^{H}\right) \in Y$ correspond to the same $\left(\omega, T_{j}^{\omega}, \tilde{\imath}^{\omega}\right) \in \tilde{Y}$. Then it is easy to see that $\tilde{\imath}^{H}=\tilde{\imath}^{H}$. Conversely, suppose that $\left(\omega, T_{j}^{\omega}, \tilde{\imath}^{\omega}\right) \in \tilde{Y}$ and $\left(\omega^{\prime}, T_{j^{\prime}}^{\omega^{\prime}}, \tilde{\imath}^{\omega^{\prime}}\right) \in \tilde{Y}$ correspond to the same $\left(T^{H}, \tilde{\imath}^{H}\right) \in Y$. Let $i^{\omega}$ be a representative for $\tilde{\imath}^{\omega}$ and $i^{H}$ a representative for $\tilde{\imath}^{H}$, then there exist $m \in M(\bar{F})$ and $h \in M_{\omega}(\bar{F})$ such that $\operatorname{Int} h\left(T_{H, 0}\right)=T_{j}^{\omega}$, Int $m\left(T_{0}\right)=T^{G}$ and

$$
i^{\omega}=\operatorname{Int} m \circ \varphi^{-1} \circ \omega \circ i_{0}^{*} \circ \operatorname{Int} h^{-1},
$$

and there exists $\omega_{H} \in \Omega\left(H, T^{H}\right)_{F}$ such that

$$
i^{H}=i^{\omega} \circ \operatorname{Int} z_{j}^{\omega} \circ \omega_{H} .
$$

Choose $i^{\prime \omega^{\prime}}, m^{\prime}, h^{\prime}$ and $\omega_{H}^{\prime}$ similarly. Then we have

$$
i_{0}^{*-1} \circ \omega^{\prime-1} \circ \varphi \circ \operatorname{Int} m^{\prime-1} m \circ \varphi^{-1} \circ \omega \circ i_{0}^{*}=\operatorname{Int} h^{\prime-1} z_{j^{\prime}}^{\omega^{\prime}} \circ \omega_{H}^{\prime} \omega_{H}^{-1} \circ \operatorname{Int} z_{j}^{\omega-1} h .
$$

Therefore,

$$
\text { Int } h^{\prime-1} z_{j^{\prime}}^{\omega^{\prime}} \circ \omega_{H}^{\prime} \omega_{H}^{-1} \circ \operatorname{Int} z_{j}^{\omega-1} h \circ\left(\omega \circ i_{0}^{*}\right)^{-1}\left(A_{M^{*}}\right)=\left(\omega^{\prime} \circ i_{0}^{*}\right)^{-1}\left(A_{M^{*}}\right) \text {. }
$$

Put $A_{1}=\left(\omega \circ i_{0}^{*}\right)^{-1}\left(A_{M^{*}}\right)$ and $A_{2}=\left(\omega^{\prime} \circ i_{0}^{*}\right)^{-1}\left(A_{M^{*}}\right)$. Then $\omega, \omega^{\prime} \in D_{M, H}$ implies that $A_{1}, A_{2} \subset$ $A_{H, 0}$. Since $H$ is quasi-split and since

$$
\operatorname{Int} h^{\prime-1} z_{j^{\prime}}^{\omega^{\prime}} \circ \omega_{H}^{\prime} \omega_{H}^{-1} \circ \operatorname{Int} z_{j}^{\omega-1} h \in \operatorname{Int} H(\bar{F}),
$$

Lemma 4.2 asserts that there exists $\omega_{H}^{\prime \prime} \in \Omega\left(H, A_{H, 0}\right)$ such that

$$
\left.\operatorname{Int} h^{\prime-1} z_{j^{\prime}}^{\omega^{\prime}} \circ \omega_{H}^{\prime} \omega_{H}^{-1} \circ \operatorname{Int} z_{j}^{\omega-1} h\right|_{A_{1}}=\left.\omega_{H}^{\prime \prime}\right|_{A_{1}} .
$$

Then

$$
\left.i_{0}^{*-1} \circ \omega^{\prime-1} \circ \varphi \circ \operatorname{Int} m^{\prime-1} m \circ \varphi^{-1} \circ \omega \circ i_{0}^{*}\right|_{A_{1}}=\left.\omega_{H}^{\prime \prime}\right|_{A_{1}} .
$$




\section{ON FUNCTORIALITY OF ZELEVINSKI INVOLUTIONS}

Since $m, m^{\prime} \in M(\bar{F})$, this shows that $\left.i_{0}^{*-1} \circ \omega^{\prime-1} \omega \circ i_{0}^{*}\right|_{A_{1}}=\left.\omega_{H}^{\prime \prime}\right|_{A_{1}}$. Therefore, we have $i_{0}^{*} \circ \omega_{H}^{\prime \prime} \circ$ $\left.i_{0}^{*-1}\right|_{\omega^{-1}\left(A_{M^{*}}\right)}=\left.\omega^{-1} \omega\right|_{\omega^{-1}\left(A_{M^{*}}\right)}$. Now, by using $\omega^{-1}\left(A_{M^{*}}\right) \subset i_{0}^{*}\left(A_{H, 0}\right)$ and $\left.i^{*}\left(\omega_{H}^{\prime \prime}\right)\right|_{i_{0}^{*}\left(A_{H, 0}\right)}=i_{0}^{*} \circ$ $\left.\omega_{H}^{\prime \prime} \circ i_{0}^{*-1}\right|_{i_{0}^{*}\left(A_{H, 0}\right)}$, we have

$$
\left.i^{*}\left(\omega_{H}^{\prime \prime}\right)\right|_{\omega^{-1}\left(A_{M^{*}}\right)}=\left.i_{0}^{*} \circ \omega_{H}^{\prime \prime} \circ i_{0}^{*-1}\right|_{\omega^{-1}\left(A_{M^{*}}\right)} .
$$

Hence, $\left.\omega^{\prime} \cdot i^{*}\left(\omega_{H}^{\prime \prime}\right) \cdot \omega^{-1}\right|_{A_{M^{*}}}=\operatorname{id}_{A_{M^{*}}}$. This implies that

$$
\omega^{\prime} \cdot i^{*}\left(\omega_{H}^{\prime \prime}\right) \cdot \omega^{-1} \in \Omega\left(M^{*}, A_{0}^{*}\right) .
$$

Therefore, Proposition 2.2 asserts that $\omega=\omega^{\prime}$. Now, by (5.2), we have

$$
\operatorname{Int} h^{\prime-1} z_{j^{\prime}}^{\omega^{\prime}} \circ \omega_{H}^{\prime} \omega_{H}^{-1} \circ \operatorname{Int} z_{j}^{\omega-1} h \in\left(\omega \circ i_{0}^{*}\right)^{-1} \circ \Omega\left(M^{*}, T_{0}^{*}\right) \circ\left(\omega \circ i_{0}^{*}\right) .
$$

On the other hand, we have Int $h^{\prime-1} z_{j^{\prime}}^{\omega^{\prime}} \circ \omega_{H}^{\prime} \omega_{H}^{-1} \circ \operatorname{Int} z_{j}^{\omega-1} h \in \Omega\left(H, T_{H, 0}\right)$. Therefore, we have $\operatorname{Int} h^{\prime-1} z_{j^{\prime}}^{\omega^{\prime}} \circ \omega_{H}^{\prime} \omega_{H}^{-1} \circ \operatorname{Int} z_{j}^{\omega-1} h \in \Omega\left(M_{\omega}, T_{H, 0}\right)$. This implies that

$$
\operatorname{Int} z_{j^{\prime}}^{\omega^{\prime}} \circ \omega_{H}^{\prime} \omega_{H}^{-1} \circ \operatorname{Int} z_{j}^{\omega-1} \in \operatorname{Int} M_{\omega}(\bar{F}) \text {. }
$$

Since Int $z_{j^{\prime}}^{\omega^{\prime}} \circ \omega_{H}^{\prime} \omega_{H}^{-1} \circ \operatorname{Int} z_{j}^{\omega-1}$ is an isomorphism from $T_{j}^{\omega}$ to $T_{j^{\prime}}^{\omega}$ defined over $F$, this shows

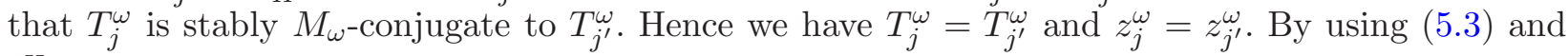
$i^{H}=i^{\omega} \circ \operatorname{Int} z_{j}^{\omega} \circ \omega_{H}=i^{\prime \omega} \circ \operatorname{Int} z_{j}^{\omega} \circ \omega_{H}^{\prime}$, we have

$$
i^{\prime \omega-1} \circ i^{\omega} \in \Omega\left(H, T_{j}^{\omega}\right)_{F} \cap \operatorname{Int} M_{\omega}(\bar{F})=\Omega\left(M_{\omega}, T_{j}^{\omega}\right)_{F} .
$$

This implies that $\tilde{\imath}^{\omega}=\tilde{\imath}^{\omega}$. Thus we have shown that

$$
\left(\omega, T_{j}^{\omega}, \widetilde{\imath}^{\omega}\right)=\left(\omega^{\prime}, T_{j^{\prime}}^{\omega^{\prime}}, \tilde{\imath}^{\omega^{\prime}}\right) .
$$

This completes the proof of Proposition 5.3.

The following theorem is an analogue of [BZ77, Lemma 2.12].

Theorem 5.6. Assume the fundamental lemma for groups and for Lie algebras. Then we have

$$
r_{M}^{G} \circ \operatorname{Tran}_{H}^{G}=\sum_{\omega \in D_{M, H}} \operatorname{Tran}_{M_{\omega}}^{M} \circ r_{M_{\omega}}^{H} .
$$

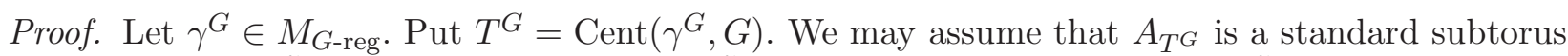
of $A_{0}$. Let $Y$ and $\tilde{Y}$ be as above. Then $Y$ and $\tilde{Y}$ are finite sets. Let $\left(\omega, T_{i j}^{\omega}, \tau^{\omega}\right) \in \tilde{Y}$, then (5.1) asserts that $\Delta_{G, H}\left(i^{\omega-1}\left(\gamma^{G}\right), \gamma^{G}\right)=\Delta_{M, M_{\omega}}\left(i^{\omega-1}\left(\gamma^{G}\right), \gamma^{G}\right)$, where $i^{\omega}$ is a representative for $\tilde{\imath}^{\omega}$. On the other hand, if $\left(T_{i}^{H}, \tilde{\imath}^{H}\right) \in Y$ corresponds to $\left(\omega, T_{i j}^{\omega}, \imath^{\omega}\right)$, then $i^{\omega-1}\left(\gamma^{G}\right)$ is stably $H$-conjugate to $i^{H^{-1}}\left(\gamma^{G}\right)$, where $i^{H}$ is a representative for $\tilde{\imath}^{H}$. Therefore,

$$
\Delta_{G, H}\left(i^{H^{-1}}\left(\gamma^{G}\right), \gamma^{G}\right)=\Delta_{M, M_{\omega}}\left(i^{\omega-1}\left(\gamma^{G}\right), \gamma^{G}\right) .
$$

Fix $a \in A_{M}^{-}$. Put $a^{*}=\varphi(a)$. Then $a^{*} \in A_{M^{*}}^{-}$. For $\omega \in D_{M, H}$, we have $i^{\omega-1}(a)=\left(\omega \circ i_{0}^{*}\right)^{-1}\left(a^{*}\right) \in$ $A_{M_{\omega}}^{-}$. Let $\theta_{H} \in \mathbb{C}[\Pi(H)]^{\text {st }}$, then by Lemmas 3.1 and 4.7 , we have

$$
\begin{aligned}
I^{M}\left(\operatorname{Tran}_{M_{\omega}}^{M} \circ r_{M_{\omega}}^{H}\left(\theta_{H}\right), a^{n} \gamma^{G}\right) & =\sum_{\left(T_{i j}^{\omega}, \tau^{\omega}\right) \in \tilde{Y}_{\omega}} \Delta_{M, M_{\omega}}\left(i^{\omega-1}\left(a^{n} \gamma^{G}\right), a^{n} \gamma^{G}\right) \cdot I^{M_{\omega}}\left(r_{M_{\omega}}^{H}\left(\theta_{H}\right), i^{\omega-1}\left(a^{n} \gamma^{G}\right)\right) \\
& =\sum_{\left(T_{i j}^{\omega}, \tau^{\omega}\right) \in \tilde{Y}_{\omega}} \Delta_{M, M_{\omega}}\left(i^{\omega-1}\left(a^{n} \gamma^{G}\right), a^{n} \gamma^{G}\right) \cdot I^{H}\left(\theta_{H}, i^{\omega-1}\left(a^{n} \gamma^{G}\right)\right)
\end{aligned}
$$

for sufficiently large $n$. Since $i^{\omega-1}\left(a^{n} \gamma^{G}\right)$ is stably $H$-conjugate to $i^{H^{-1}}\left(a^{n} \gamma^{G}\right)$, we have

$$
I^{H}\left(\theta_{H}, i^{\omega-1}\left(a^{n} \gamma^{G}\right)\right)=I^{H}\left(\theta_{H}, i^{H^{-1}}\left(a^{n} \gamma^{G}\right)\right) .
$$




\section{K. HiRAGA}

Therefore, by Lemmas 3.1 and 4.7 and Proposition 5.3, we have

$$
\begin{aligned}
I^{M}\left(r_{M}^{G} \circ \operatorname{Tran}_{H}^{G}\left(\theta_{H}\right), a^{n} \gamma^{G}\right) & =I^{G}\left(\operatorname{Tran}_{H}^{G}\left(\theta_{H}\right), a^{n} \gamma^{G}\right), \\
& =\sum_{\left(T_{i}^{H}, \tilde{\imath}^{H}\right) \in Y} \Delta_{G, H}\left(i^{H^{-1}}\left(a^{n} \gamma^{G}\right), a^{n} \gamma^{G}\right) \cdot I^{H}\left(\theta_{H}, i^{H-1}\left(a^{n} \gamma^{G}\right)\right), \\
& =\sum_{\omega \in D_{M, H}} \sum_{\left(T_{i j}^{\omega}, \tilde{\imath}^{\omega}\right) \in \tilde{Y}_{\omega}} \Delta_{M, M_{\omega}}\left(i^{\omega-1}\left(a^{n} \gamma^{G}\right), a^{n} \gamma^{G}\right) \cdot I^{H}\left(\theta_{H}, i^{\omega-1}\left(a^{n} \gamma^{G}\right)\right), \\
& =\sum_{\omega \in D_{M, H}} I^{M}\left(\operatorname{Tran}_{M_{\omega}}^{M} \circ r_{M_{\omega}}^{H}\left(\theta_{H}\right), a^{n} \gamma^{G}\right),
\end{aligned}
$$

for sufficiently large $n$. It is then immediate from Lemma 3.2 that

$$
I^{M}\left(r_{M}^{G} \circ \operatorname{Tran}_{H}^{G}\left(\theta_{H}\right), \gamma^{G}\right)=\sum_{\omega \in D_{M, H}} I^{M}\left(\operatorname{Tran}_{M_{\omega}}^{M} \circ r_{M_{\omega}}^{H}\left(\theta_{H}\right), \gamma^{G}\right)
$$

\section{Commutativity}

By Corollary 3.4 and Proposition 4.6, we can define $\mathbf{D}_{G} \circ \operatorname{Tran}_{H}^{G}$ and $\operatorname{Tran}_{H}^{G} \circ \mathbf{D}_{H}$. By Lemma 5.1, we may regard $\left(M_{\omega}, \mathcal{M}_{\omega}, s_{\omega}, \xi_{\omega}\right)$ as a set of endoscopic data for $G$. Let $\Delta_{G, M_{\omega}}$ be the LanglandsShelstad transfer factor. If $\Gamma\left(M_{\omega}, M\right)=\emptyset$, then we can show that $\Delta_{G, M_{\omega}} \equiv 0$. If $\Gamma\left(M_{\omega}, M\right) \neq \emptyset$, then we normalize $\Delta_{G, M_{\omega}}$ so that

$$
\Delta_{G, M_{\omega}}\left(\gamma^{M_{\omega}}, \gamma^{M}\right)=\Delta_{M, M_{\omega}}\left(\gamma^{M_{\omega}}, \gamma^{M}\right),
$$

for all $\left(\gamma^{M_{\omega}}, \gamma^{M}\right) \in \Gamma\left(M_{\omega}, M\right)$. (As in Lemma 5.2, we can show that the relative transfer factors $\Delta_{G, M_{\omega}}$ and $\Delta_{M, M_{\omega}}$ are equal on $\Gamma\left(M_{\omega}, M\right)$.) Then it is not difficult to show that

$$
i_{M}^{G} \circ \operatorname{Tran}_{M_{\omega}}^{M}=\operatorname{Tran}_{M_{\omega}}^{G}
$$

and that

$$
\operatorname{Tran}_{H}^{G} \circ i_{M_{\omega}}^{H}=\operatorname{Tran}_{M_{\omega}}^{G}
$$

Thus we have the following lemma.

LEMma 6.1. We have

$$
i_{M}^{G} \circ \operatorname{Tran}_{M_{\omega}}^{M}=\operatorname{Tran}_{H}^{G} \circ i_{M_{\omega}}^{H}
$$

Recall that $M_{0}$ is a minimal Levi subgroup of $G$. We put $M_{0}^{*}=\varphi\left(M_{0}\right) \in \mathcal{L}^{G^{*}}$.

Lemma 6.2. The standard Levi subgroup $M_{0}^{*}$ itself is the only associate standard Levi subgroup of $M_{0}^{*}$.

Proof. Put $X_{*}\left(A_{M_{0}^{*}}\right)=\operatorname{Hom}\left(\mathbb{G}_{m}, A_{M_{0}^{*}}\right)$ and $\mathfrak{a}_{M_{0}^{*}}=X_{*}\left(A_{M_{0}^{*}}\right) \otimes_{\mathbb{Z}} \mathbb{R}$. Let $\omega \in \Omega\left(G, A_{0}\right)$, then Lemma 4.2 asserts that there exists $\omega^{*} \in \Omega\left(G^{*}, A_{0}^{*}\right)$ such that $\omega=\operatorname{res}_{A_{0}}\left(\varphi^{-1} \circ \omega^{*} \circ \varphi\right)$. This implies that for each chamber $C$ of $\mathfrak{a}_{M_{0}^{*}}$, there exists

$$
\omega^{*} \in \Omega\left(G^{*}, A_{0}^{*}\right)_{\mathfrak{a}_{M_{0}^{*}}}=\left\{\omega^{\prime} \in \Omega\left(G^{*}, A_{0}^{*}\right) \mid \omega^{\prime}\left(\mathfrak{a}_{M_{0}^{*}}\right)=\mathfrak{a}_{M_{0}^{*}}\right\}
$$

such that $\omega^{*}(C)=C_{M_{0}^{*}}^{+}$and $\omega^{*}\left(S\left(M_{0}^{*}\right)\right)=S\left(M_{0}^{*}\right)$. Hence Lemma 2 shows that $M_{0}^{*}$ itself is the only associate standard Levi subgroup of $M_{0}^{*}$.

Therefore, Lemma 2.5 implies the following lemma. 


\section{ON FUNCTORIALITY OF ZELEVINSKI INVOLUTIONS}

Lemma 6.3. If $M^{*} \in \mathcal{L}^{G^{*}}$ satisfies $\omega\left(A_{M^{*}}\right) \subset A_{M_{0}^{*}}$ for some $\omega \in \Omega\left(G^{*}, A_{0}^{*}\right)$, then we have

$$
M^{*} \supset M_{0}^{*} \text {. }
$$

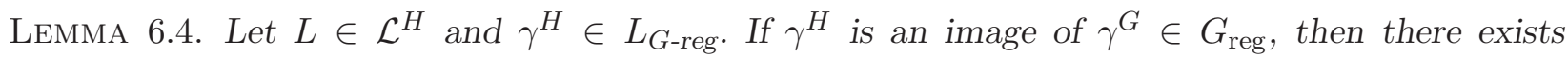
$\omega \in \Omega\left(G^{*}, A_{0}^{*}\right)$ such that

$$
\omega \circ i_{0}^{*}\left(A_{L}\right) \subset \varphi\left(A_{0}\right)=A_{M_{0}^{*}} .
$$

Proof. Let $T^{H}=\operatorname{Cent}\left(\gamma^{H}, H\right)$ and $T^{G}=\operatorname{Cent}\left(\gamma^{G}, G\right)$. We may assume that $A_{T^{H}}$ is a standard subtorus of $A_{H, 0}$ and $A_{T^{G}}$ is a standard subtorus of $A_{0}$. Put $M_{T^{H}}=\operatorname{Cent}\left(A_{T^{H}}, H\right) \in \mathcal{L}^{H}$ and $M=M_{T^{G}}=\operatorname{Cent}\left(A_{T^{G}}, G\right) \in \mathcal{L}^{G}$. Since $\gamma^{H}$ is an image of $\gamma^{G}$, there exists $i^{H} \in I^{G, H}\left(T^{H}, T^{G}\right)$ such that $i^{H}\left(\gamma^{H}\right)=\gamma^{G}$. Then Lemma 5.5 asserts that there exist $\omega_{H} \in \Omega\left(H, T_{H, 0}\right)_{F}, \omega \in D_{M, H}$, $\omega_{M} \in \Omega\left(M, T_{0}\right), m \in M(\bar{F})$ and $h \in M_{T^{H}}(\bar{F})$ such that $\operatorname{Int} m\left(T_{0}\right)=T^{G}$, Int $h\left(T_{H, 0}\right)=T^{H}$ and

$$
i^{H}=\operatorname{Int} m \circ \omega_{M} \circ \varphi^{-1} \circ \omega \circ i_{0}^{*} \circ \omega_{H} \circ \operatorname{Int} h^{-1} .
$$

Hence, we have $A_{T^{G}}=i^{H}\left(A_{T^{H}}\right)=\varphi^{-1} \circ \omega \circ i_{0}^{*} \circ \omega_{H}\left(A_{T^{H}}\right)$. By using $i_{0}^{*} \circ \omega_{H} \circ i_{0}^{*-1} \in i^{*}\left(\omega_{H}\right)$. $\Omega\left(M^{H}, T_{0}^{*}\right)$ and $i_{0}^{*}\left(A_{T^{H}}\right) \subset i_{0}^{*}\left(A_{H, 0}\right)$, we have $\varphi^{-1} \circ \omega \circ i_{0}^{*} \circ \omega_{H}\left(A_{T^{H}}\right)=\varphi^{-1} \circ \omega \cdot i^{*}\left(\omega_{H}\right) \circ i_{0}^{*}\left(A_{T^{H}}\right)$. Thus

$$
\omega \cdot i^{*}\left(\omega_{H}\right) \circ i_{0}^{*}\left(A_{L}\right) \subset \omega \cdot i^{*}\left(\omega_{H}\right) \circ i_{0}^{*}\left(A_{T^{H}}\right)=\varphi\left(A_{T^{G}}\right) \subset \varphi\left(A_{0}\right) .
$$

For $M^{*} \in \mathcal{L}^{G^{*}}$ and $L \in \mathcal{L}^{H}$, we define $D_{M^{*}, H, L}$ as in $\oint 2$.

Corollary 6.5. Let $M^{*} \in \mathcal{L}^{G^{*}}$. If $D_{M^{*}, H, L} \neq \emptyset$ and if there exists $\gamma^{H} \in L_{G \text {-reg }}$ that is an image of an element $\gamma^{G} \in G_{\text {reg }}$, then we have

$$
M^{*} \supset M_{0}^{*} .
$$

Proof. Let $\omega \in D_{M^{*}, H, L}$, then we have $\omega^{-1}\left(A_{M^{*}}\right) \subset i_{0}^{*}\left(A_{L}\right)$. On the other hand, Lemma 6.4 asserts that there exists $\omega^{\prime} \in \Omega\left(G^{*}, A_{0}^{*}\right)$ such that $\omega^{\prime} \circ i_{0}^{*}\left(A_{L}\right) \subset A_{M_{0}^{*}}$. Thus we have $\omega^{\prime} \omega^{-1}\left(A_{M^{*}}\right) \subset A_{M_{0}^{*}}$. It is then immediate from Lemma 6.3 that $M^{*} \supset M_{0}^{*}$.

We put $a(G)=\operatorname{dim} A_{0}, a\left(G^{*}\right)=\operatorname{dim} A_{0}^{*}$ and $a(H)=\operatorname{dim} A_{H, 0}$. Then for $M \in \mathcal{L}^{G}$, we have $r(\varphi(M))-r(M)=a\left(G^{*}\right)-a(G)$. We put

$$
a_{M^{*}, H, L}=\sharp D_{M^{*}, H, L}
$$

as in $\S 2$.

Theorem 6.6. Assume the fundamental lemma for groups and for Lie algebras. Then we have

$$
\mathbf{D}_{G} \circ \operatorname{Tran}_{H}^{G}=(-1)^{a(G)-a(H)} \operatorname{Tran}_{H}^{G} \circ \mathbf{D}_{H} .
$$

Proof. By Theorem 5.6, we have

$$
\begin{aligned}
\mathbf{D}_{G} \circ \operatorname{Tran}_{H}^{G} & =\sum_{M \in \mathcal{L}^{G}}(-1)^{r(M)} i_{M}^{G} \circ r_{M}^{G} \circ \operatorname{Tran}_{H}^{G} \\
& =\sum_{M \in \mathcal{L}^{G}} \sum_{\omega \in D_{M, H}}(-1)^{r(M)} i_{M}^{G} \circ \operatorname{Tran}_{M_{\omega}}^{M} \circ r_{M_{\omega}}^{H} .
\end{aligned}
$$




\section{K. HiRAGA}

Since $i_{M}^{G} \circ \operatorname{Tran}_{M_{\omega}}^{M}=\operatorname{Tran}_{H}^{G} \circ i_{M_{\omega}}^{H}$, this is equal to

$$
\begin{aligned}
\sum_{M \in \mathcal{L}^{G}} & \sum_{\omega \in D_{M, H}}(-1)^{r(M)} \operatorname{Tran}_{H}^{G} \circ i_{M_{\omega}}^{H} \circ r_{M_{\omega}}^{H}, \\
= & (-1)^{a(G)-a\left(G^{*}\right)} \sum_{\substack{M^{*} \in \mathcal{L}^{G^{*}} \\
M^{*} \supset M_{0}^{*}}}(-1)^{r\left(M^{*}\right)} \sum_{L \in \mathcal{L}^{H}} \sum_{\omega \in D_{M^{*}, H, L}} \operatorname{Tran}_{H}^{G} \circ i_{L}^{H} \circ r_{L}^{H}, \\
= & (-1)^{a(G)-a\left(G^{*}\right)} \sum_{\substack{M^{*} \in \mathcal{L}^{G^{*}} \\
M^{*} \supset M_{0}^{*}}}(-1)^{r\left(M^{*}\right)} \sum_{L \in \mathcal{L}^{H}} a_{M^{*}, H, L} \operatorname{Tran}_{H}^{G} \circ i_{L}^{H} \circ r_{L}^{H} .
\end{aligned}
$$

By using Corollary 6.5 and Theorem 2.4, we can show that this is equal to

$$
\begin{aligned}
& (-1)^{a(G)-a\left(G^{*}\right)} \sum_{L \in \mathcal{L}^{H}} \sum_{M^{*} \in \mathcal{L}^{G^{*}}}(-1)^{r\left(M^{*}\right)} a_{M^{*}, H, L} \operatorname{Tran}_{H}^{G} \circ i_{L}^{H} \circ r_{L}^{H} \\
& =(-1)^{a(G)-a\left(G^{*}\right)}(-1)^{a\left(G^{*}\right)-a(H)} \sum_{L \in \mathcal{L}^{H}}(-1)^{r(L)} \operatorname{Tran}_{H}^{G} \circ i_{L}^{H} \circ r_{L}^{H} \\
& =(-1)^{a(G)-a(H)} \operatorname{Tran}_{H}^{G} \circ \mathbf{D}_{H} .
\end{aligned}
$$

Now, we treat the general case. Let $\left(H_{1}, \xi_{H_{1}}\right)$ be a $z$-pair for the set of endoscopic data $(H, \mathcal{H}, s, \xi)$ as in $\S 5$. Then for $\omega \in D_{M, H}$, we can define a $z$-pair $\left(M_{\omega, 1}, \xi_{H_{1}}\right)$ for $\left(M_{\omega}, \mathcal{M}_{\omega}, s_{\omega}, \xi_{\omega}\right)$ as in $\S 5$. Let $Z_{1}$ be the kernel of the morphism $H_{1} \longrightarrow H$ and $\lambda_{H_{1}}$ the quasi-character of $Z_{1}(F)$ defined in [KS99, $\S 2.2]$. Let $\Pi\left(H_{1}, \lambda_{H_{1}}\right)$ be the set of equivalence classes of irreducible admissible representations of $H_{1}(F)$ whose central characters on $Z_{1}(F)$ are equal to $\lambda_{H_{1}}$. We denote by $\mathbb{C}\left[\Pi\left(H_{1}, \lambda_{H_{1}}\right)\right]$ the subspace of $\mathbb{C}\left[\Pi\left(H_{1}\right)\right]$ generated by $\Pi\left(H_{1}, \lambda_{H_{1}}\right)$. We put $\mathbb{C}\left[\Pi\left(H_{1}, \lambda_{H_{1}}\right)\right]^{\mathrm{st}}=\mathbb{C}\left[\Pi\left(H_{1}, \lambda_{H_{1}}\right)\right] \cap \mathbb{C}\left[\Pi\left(H_{1}\right)\right]^{\mathrm{st}}$. Then we have

$$
\operatorname{Tran}_{H_{1}}^{G}\left(\mathbb{C}\left[\Pi\left(H_{1}, \lambda_{H_{1}}\right)\right]^{\mathrm{st}}\right) \subset \mathbb{C}[\Pi(G)],
$$

as in Proposition 4.6. On the other hand, by [KS99, Lemma 5.1.C] we have

$$
\Delta_{G, H_{1}}\left(z \gamma^{H_{1}}, \gamma^{G}\right) \cdot I^{H_{1}}\left(\theta_{H_{1}}, z \gamma^{H_{1}}\right)=\Delta_{G, H_{1}}\left(\gamma^{H_{1}}, \gamma^{G}\right) \cdot I^{H_{1}}\left(\theta_{H_{1}}, \gamma^{H_{1}}\right),
$$

for all $z \in Z_{1}(F)$ and $\theta_{H_{1}} \in \mathbb{C}\left[\Pi\left(H_{1}, \lambda_{H_{1}}\right)\right]^{\text {st }}$. Therefore, by arguments similar to the proofs of Theorems 5.6 and 6.6, we have the following theorem.

Theorem 6.7. Assume the fundamental lemma for groups and for Lie algebras. Then we have

$$
\begin{gathered}
r_{M}^{G} \circ \operatorname{Tran}_{H_{1}}^{G}=\sum_{\omega \in D_{M, H}} \operatorname{Tran}_{M_{\omega, 1}}^{M} \circ r_{M_{\omega, 1}}^{H_{1}}, \\
\mathbf{D}_{G} \circ \operatorname{Tran}_{H_{1}}^{G}=(-1)^{a(G)-a(H)} \operatorname{Tran}_{H_{1}}^{G} \circ \mathbf{D}_{H_{1}} .
\end{gathered}
$$

\section{Functoriality}

In this section, we discuss the relation between the Zelevinski involutions and the Arthur conjecture [Art89, Conjecture 6.1]. So we assume [Art89, Conjecture 6.1], Hypothesis 1.1 and the fundamental lemma for groups and for Lie algebras in this section. Let

$$
\psi: W_{F} \times S U_{2}(\mathbb{C}) \times S L_{2}(\mathbb{C}) \longrightarrow{ }^{L} G
$$

be an Arthur parameter and let $\Pi_{\psi}(G)$ be the $A$-packet of $\psi$. We denote the second factor by $S U_{2}(\mathbb{C})$ in order to distinguish this from the third factor $S L_{2}(\mathbb{C})$, which is used to define the Arthur 


\section{ON FUNCTORIALITY OF ZELEVINSKI INVOLUTIONS}

parameters in [Art89]. We put

$$
\begin{aligned}
& S_{\psi}=\operatorname{Cent}(\psi, \hat{G}), \\
& \mathbb{S}_{\psi}=S_{\psi} / S_{\psi}^{0} .
\end{aligned}
$$

Let $s_{\psi}=\psi(1 \times 1 \times(-1)) \in S_{\psi}$. We denote by $\mathbb{C}\left[\Pi_{\psi}(G)\right]$ the subspace generated by $\Pi_{\psi}(G)$. We put $\mathbb{C}\left[\Pi_{\psi}(G)\right]^{\text {st }}=\mathbb{C}\left[\Pi_{\psi}(G)\right] \cap \mathbb{C}[\Pi(G)]^{\text {st }}$. For the set of endoscopic data $(H, \mathcal{H}, s, \xi)$ corresponding to a semisimple element $s \in S_{\psi}$, we choose a $z$-pair $\left(H_{1}, \xi_{H_{1}}\right)$. Then $\xi_{H_{1}} \circ \psi$ is an Arthur parameter on $H_{1}$, which we also denote by $\psi$. Let $\theta_{\psi}^{H_{1}} \in \mathbb{C}\left[\Pi_{\psi}\left(H_{1}\right)\right]^{\text {st }}$ be the distribution $\psi_{H}$ in [Art89, Conjecture 6.1]. As in [Art89, Conjecture 6.1], we define the function $\delta$. Then

$$
\operatorname{Tran}_{H_{1}}^{G}\left(\theta_{\psi}^{H_{1}}\right)=\sum_{\pi \in \Pi_{\psi}(G)} \delta\left(s_{\psi} s, \pi\right) \cdot \pi
$$

where $H$ is the endoscopic group corresponding to $s \in S_{\psi}$. Let $\rho=\rho_{\psi}$ be the normalizing function in [Art89, Conjecture 6.1]. Then

$$
\langle\bar{s}, \pi \mid \rho\rangle=\delta(s, \pi) \rho(s)^{-1},
$$

where $\bar{s} \in \mathbb{S}_{\psi}$ is the image of $s \in S_{\psi}$. We identify $S U_{2}(\mathbb{C})$ with $S L_{2}(\mathbb{C})$. We define $d(\psi)$ by

$$
d(\psi)(w \times t \times u)=\psi(w \times u \times t), \quad w \times t \times u \in W_{F} \times S U_{2}(\mathbb{C}) \times S L_{2}(\mathbb{C}) .
$$

Then $d(\psi)$ is the Arthur parameter constructed from $\psi$ by interchanging the role of $S U_{2}(\mathbb{C})$ and $S L_{2}(\mathbb{C})$. We have $S_{\psi}=S_{d(\psi)}$ and $\mathbb{S}_{\psi}=\mathbb{S}_{d(\psi)}$. If $G$ is quasi-split, then we put $G^{*}=G$ and

$$
\mathbb{S}_{\psi}^{*}=S_{\psi} / S_{\psi}^{0} \cdot Z_{\hat{G}}^{\Gamma} \text {. }
$$

We fix Whittaker data $\chi$ for $G^{*}$ (see $[$ KS99, $\S 5.3]$ ). Let $\phi_{\psi}$ be the corresponding Langlands parameter on $G^{*}$ and $\Pi_{\phi_{\psi}}\left(G^{*}\right)$ the $L$-packet of $\phi_{\psi}$. We determine the base point $\pi_{\chi} \in \Pi_{\phi_{\psi}}\left(G^{*}\right)$ as in $[$ Art89, $\S 6]$. Let $(H, \mathcal{H}, s, \xi)$ be the set of endoscopic data corresponding to $s \in S_{\psi}$. If $z \in Z_{\hat{G}}^{\Gamma}$, then $(H, \mathcal{H}, s, \xi)$ and $(H, \mathcal{H}, s z, \xi)$ are equivalent endoscopic data. Therefore, Hypothesis 1.1 implies that for $\pi \in \Pi_{\psi}\left(G^{*}\right)$,

$$
\left\langle\bar{s}, \pi \mid \pi_{\chi}\right\rangle=\delta(s, \pi) \delta\left(s, \pi_{\chi}\right)^{-1}
$$

depends only on the image of $s$ in $\mathbb{S}_{\psi}^{*}$. (We also write $\bar{s}$ for the image of $s$ in $\mathbb{S}_{\psi}^{*}$.) Thus we may regard $\left\langle\cdot, \pi \mid \pi_{\chi}\right\rangle$ as an irreducible character of $\mathbb{S}_{\psi}^{*}$.

Let $\{M\}$ be the set of associate standard Levi subgroups of $M$. We say that $\pi \in \Pi(G)$ is of type $\{M\}$ if $r_{M}^{G}(\pi)$ is a non-zero linear combination of supercuspidal representations of $M(F)$. If $\pi$ is of type $\{M\}$, then we put $r_{\pi}=r(M)$. For $\pi \in \Pi(G)$, we define $\mathbf{d}_{G}(\pi)$ by

$$
\mathbf{d}_{G}(\pi)=(-1)^{r_{\pi}} \mathbf{D}_{G}(\pi) .
$$

Then we have $r_{\pi}=r_{\mathbf{d}_{G}(\pi)}$. The following proposition, which is conjectured by Kato [Kat93], is proved by Aubert [Aub95, Aub96].

Proposition 7.1. For $\pi \in \Pi(G)$, we have $\mathbf{d}_{G}(\pi) \in \Pi(G)$.

Lemma 7.2. Conjecture 1.4 implies Conjecture 1.2.

Proof. First, we treat the case that $G$ is quasi-split. Put $G=G^{*}$. We prove

$$
\mathbf{d}_{G^{*}}\left(\Pi_{\psi}\left(G^{*}\right)\right)=\Pi_{d(\psi)}\left(G^{*}\right)
$$

by induction on $r\left(G^{*}\right)$. If $\mathbb{S}_{\psi}^{*}=\{1\}$, then there is nothing to prove. Suppose that $\mathbb{S}_{\psi}^{*} \neq\{1\}$. Let $\pi \in \Pi_{\psi}\left(G^{*}\right)$, then since $\left\langle\cdot, \pi \mid \pi_{\chi}\right\rangle$ is an irreducible character, there exists $\bar{s} \in \mathbb{S}_{\psi}^{*}$ such that $\bar{s} \neq \overline{1}$ and $\left\langle\bar{s}, \pi \mid \pi_{\chi}\right\rangle \neq 0$. Let $(H, \mathcal{H}, s, \xi)$ be the set of endoscopic data corresponding to $s \in S_{\psi}$. Choose a $z$-pair $\left(H_{1}, \xi_{H_{1}}\right)$. Then the coefficient of $\pi$ in $\operatorname{Tran}_{H_{1}}^{G^{*}}\left(\theta_{\psi}^{H_{1}}\right)$ is not zero. On the other 


\section{K. HiragA}

hand, by the inductive assumption, we have $\mathbf{d}_{H_{1}}\left(\Pi_{\psi}\left(H_{1}\right)\right)=\Pi_{d(\psi)}\left(H_{1}\right)$. Hence, Corollary 3.4 asserts that $\mathbf{D}_{H_{1}}\left(\theta_{\psi}^{H_{1}}\right) \in \mathbb{C}\left[\Pi_{d(\psi)}\left(H_{1}\right)\right]^{\text {st }}$. Therefore, Hypothesis 1.1 implies that there exists $c_{\psi}^{H_{1}} \in \mathbb{C}^{\times}$ such that

By Theorem 6.7, we have

$$
\mathbf{D}_{H_{1}}\left(\theta_{\psi}^{H_{1}}\right)=c_{\psi}^{H_{1}} \theta_{d(\psi)}^{H_{1}}
$$

$$
\mathbf{D}_{G^{*}} \circ \operatorname{Tran}_{H_{1}}^{G^{*}}\left(\theta_{\psi}^{H_{1}}\right)=(-1)^{a\left(G^{*}\right)-a(H)} c_{\psi}^{H_{1}} \operatorname{Tran}_{H_{1}}^{G^{*}}\left(\theta_{d(\psi)}^{H_{1}}\right) .
$$

Since the coefficient of $\pi$ in $\operatorname{Tran}_{H_{1}}^{G^{*}}\left(\theta_{\psi}^{H_{1}}\right)$ is not zero, this shows that the coefficient of $\mathbf{d}_{G^{*}}(\pi)$ in $\operatorname{Tran}_{H_{1}}^{G^{*}}\left(\theta_{d(\psi)}^{H_{1}}\right)$ is not zero. Therefore, $\mathbf{d}_{G^{*}}(\pi) \in \Pi_{d(\psi)}\left(G^{*}\right)$. Thus we have $\mathbf{d}_{G^{*}}\left(\Pi_{\psi}\left(G^{*}\right)\right)=\Pi_{d(\psi)}\left(G^{*}\right)$.

Now, we turn to the general case. Let $\pi \in \Pi(G)$, then [Art89, Conjecture 6.1(iii)] shows that we have $\pi \in \Pi_{\psi}(G)$ if and only if the coefficient of $\pi$ in $\operatorname{Tran}_{G^{*}}^{G}\left(\theta_{\psi}^{G^{*}}\right)$ is not zero. By Theorem 6.6, we have

$$
\mathbf{D}_{G} \circ \operatorname{Tran}_{G^{*}}^{G}\left(\theta_{\psi}^{G^{*}}\right)=(-1)^{a\left(G^{*}\right)-a(G)} c_{\psi}^{G^{*}} \operatorname{Tran}_{G^{*}}^{G}\left(\theta_{d(\psi)}^{G^{*}}\right) .
$$

Therefore, $\mathbf{d}_{G}\left(\Pi_{\psi}(G)\right)=\Pi_{d(\psi)}(G)$.

Now, we assume Conjecture 1.4. By comparing the coefficient of $\mathbf{d}_{G}(\pi)$ in

$$
\mathbf{D}_{G} \circ \operatorname{Tran}_{H_{1}}^{G}\left(\theta_{\psi}^{H_{1}}\right)=(-1)^{a(G)-a(H)} c_{\psi}^{H_{1}} \operatorname{Tran}_{H_{1}}^{G}\left(\theta_{d(\psi)}^{H_{1}}\right),
$$

we have

$$
\delta\left(s_{\psi} s, \pi\right)(-1)^{r_{\pi}}=(-1)^{a(G)-a(H)} c_{\psi}^{H_{1}} \delta_{d}\left(s_{d(\psi)} s, \mathbf{d}_{G}(\pi)\right),
$$

where $\delta_{d}=\delta_{d(\psi)}$. We assume that $G=G^{*}$. By dividing the formula (7.1) by that of $\pi_{\chi}$, we have

$$
\left\langle\bar{s}_{\psi} \bar{s}, \pi \mid \pi_{\chi}\right\rangle(-1)^{r_{\pi}-r_{\pi_{\chi}}}=\left\langle\bar{s}_{d(\psi)} \bar{s}, \mathbf{d}_{G^{*}}(\pi) \mid \pi_{d, \chi}\right\rangle\left\langle\bar{s}_{d(\psi)} \bar{s}, \mathbf{d}_{G^{*}}\left(\pi_{\chi}\right) \mid \pi_{d, \chi}\right\rangle^{-1},
$$

where $\pi_{d, \chi}$ is the base point in $\Pi_{d(\psi)}\left(G^{*}\right)$. Since $\delta\left(s_{\psi} s, \pi_{\chi}\right) \neq 0$, we have $\delta_{d}\left(s_{d(\psi)} s, \mathbf{d}_{G^{*}}\left(\pi_{\chi}\right)\right) \neq 0$ and

$$
\left\langle\bar{s}_{d(\psi)} \bar{s}, \mathbf{d}_{G^{*}}\left(\pi_{\chi}\right) \mid \pi_{d, \chi}\right\rangle \neq 0
$$

for all $s \in S_{d(\psi)}$. If we put $\bar{s}=\bar{s}_{\psi}$ and $\pi=\mathbf{d}_{G^{*}}\left(\pi_{d, \chi}\right)$, then we have

$$
\left\langle\overline{1}, \mathbf{d}_{G^{*}}\left(\pi_{d, \chi}\right) \mid \pi_{\chi}\right\rangle(-1)^{r_{\pi_{d, \chi}}-r_{\pi_{\chi}}}=\left\langle\bar{s}_{d(\psi)} \bar{s}_{\psi}, \mathbf{d}_{G^{*}}\left(\pi_{\chi}\right) \mid \pi_{d, \chi}\right\rangle^{-1} .
$$

Since $\bar{s}_{d(\psi)} \bar{s}_{\psi}$ is an element of order 1 or 2 contained in the center of $\mathbb{S}_{d(\psi)}$, this implies that

$$
\left\langle\overline{1}, \mathbf{d}_{G^{*}}\left(\pi_{d, \chi}\right) \mid \pi_{\chi}\right\rangle=\left\langle\overline{1}, \mathbf{d}_{G^{*}}\left(\pi_{\chi}\right) \mid \pi_{d, \chi}\right\rangle=1 .
$$

Thus we have the following lemma.

Lemma 7.3. The character $\left\langle\cdot, \mathbf{d}_{G^{*}}\left(\pi_{\chi}\right) \mid \pi_{d, \chi}\right\rangle$ is a one-dimensional character of $\mathbb{S}_{d(\psi)}$.

Moreover, since

$$
\left\langle\bar{s}, \pi \mid \pi_{\chi}\right\rangle= \pm\left\langle\bar{s}, \mathbf{d}_{G^{*}}(\pi) \mid \pi_{d, \chi}\right\rangle\left\langle\bar{s}, \mathbf{d}_{G^{*}}\left(\pi_{\chi}\right) \mid \pi_{d, \chi}\right\rangle^{-1}
$$

we have the following proposition.

Proposition 7.4. Let $G=G^{*}$. Assume the fundamental lemma for groups and for Lie algebras, the Arthur conjecture [Art89, Conjecture 6.1], Hypothesis 1.1 and Conjecture 1.4. Then

$$
\left\langle\bar{s}, \pi \mid \pi_{\chi}\right\rangle=\left\langle\bar{s}, \mathbf{d}_{G^{*}}(\pi) \mid \pi_{d, \chi}\right\rangle\left\langle\bar{s}, \mathbf{d}_{G^{*}}\left(\pi_{\chi}\right) \mid \pi_{d, \chi}\right\rangle^{-1} .
$$

This is the formula in Conjecture 1.3. By an easy calculation, we have

$$
\mathbf{D}_{G^{*}}\left(\sum_{\pi \in \Pi_{\psi}\left(G^{*}\right)}\left\langle\bar{s}_{\psi}, \pi \mid \pi_{\chi}\right\rangle \cdot \pi\right)=(-1)^{r_{\pi_{\chi}}}\left\langle\bar{s}_{d(\psi)}, \mathbf{d}_{G^{*}}\left(\pi_{\chi}\right) \mid \pi_{d, \chi}\right\rangle^{-1}\left(\sum_{\pi \in \Pi_{d(\psi)}\left(G^{*}\right)}\left\langle\bar{s}_{d(\psi)}, \pi \mid \pi_{d, \chi}\right\rangle \cdot \pi\right) .
$$


Since $\theta_{\psi}^{G^{*}}=\sum_{\pi \in \Pi_{\psi}\left(G^{*}\right)} \delta\left(s_{\psi}, \pi\right) \cdot \pi$, the relation $\mathbf{D}_{G^{*}}\left(\theta_{\psi}^{G^{*}}\right)=c_{\psi}^{G^{*}} \theta_{d(\psi)}^{G^{*}}$ implies that

$$
c_{\psi}^{G^{*}}=(-1)^{r_{\pi_{\chi}}} \delta\left(s_{\psi}, \pi_{\chi}\right) \delta_{d}\left(s_{d(\psi)}, \pi_{d, \chi}\right)^{-1}\left\langle\bar{s}_{d(\psi)}, \mathbf{d}_{G^{*}}\left(\pi_{\chi}\right) \mid \pi_{d, \chi}\right\rangle^{-1} .
$$

Therefore, by using $\left|\delta\left(s_{\psi}, \pi_{\chi}\right)\right|=1$ and $\left|\delta_{d}\left(s_{d(\psi)}, \pi_{d, \chi}\right)\right|=1$ (see [Art89, Conjecture 6.1 (iv)]), we have $\left|c_{\psi}^{G^{*}}\right|=1$.

By dividing (7.1) by $\rho\left(s_{\psi} s\right)$, we have

$$
\left\langle\bar{s}_{\psi} \bar{s}, \pi \mid \rho\right\rangle(-1)^{r_{\pi}}=(-1)^{a(G)-a(H)} c_{\psi}^{H_{1}} \rho\left(s_{\psi} s\right)^{-1} \rho_{d}\left(s_{d(\psi)} s\right)\left\langle\bar{s}_{d(\psi)} \bar{s}, \mathbf{d}_{G}(\pi) \mid \rho_{d}\right\rangle .
$$

Hence, by $\left|c_{\psi}^{H_{1}}\right|=1$ and [Art89, Conjecture 6.1 (iii)], we have

$$
\langle\overline{1}, \pi \mid \rho\rangle=\left\langle\overline{1}, \mathbf{d}_{G}(\pi) \mid \rho_{d}\right\rangle .
$$

\section{Appendix. Proofs of the results in $\S 2$}

In this section, we prove the results in $\S 2$. We keep the notation in $\S 2$. We begin with the following lemma.

Lemma A.1. If $\alpha \in R^{+}\left(G ; \mathfrak{a}_{M}\right)$ is $R(G)$-symmetric, then there exist

$$
\tilde{\alpha}_{1}, \ldots, \tilde{\alpha}_{r} \in R^{+}(G)
$$

satisfying the following conditions:

1) $\left(\tilde{\alpha}_{i}, \tilde{\alpha}_{j}\right)=0$, if $i \neq j$;

2) $\operatorname{res}_{\mathfrak{a}_{M}}\left(\tilde{\alpha}_{i}\right) \in \mathbb{R}_{+}^{\times} \alpha, \quad i=1, \ldots, r$;

3) $s_{\tilde{\alpha}_{1}} \cdots s_{\tilde{\alpha}_{r}} \in \Omega(G)_{\mathfrak{a}_{M}}$;

4) $s_{\alpha}=\operatorname{res}_{\mathfrak{a}_{M}}\left(s_{\tilde{\alpha}_{1}} \cdots s_{\tilde{\alpha}_{r}}\right)$.

Before proving Lemma 1, we prepare some lemmas. For $S\left(M^{\prime}\right) \subset S(G)$, we put

$$
C_{M^{\prime}}^{+}=\left\{a \in \mathfrak{a}_{M^{\prime}} \mid \tilde{\alpha}(a)>0 \text { for all } \tilde{\alpha} \in S(G)-S\left(M^{\prime}\right)\right\} .
$$

We denote by $\overline{C_{M^{\prime}}^{+}}$the closure of $C_{M^{\prime}}^{+}$. For $S\left(M^{\prime}\right), S\left(M^{\prime \prime}\right) \subset S(G)$, we put

$$
\Omega\left(M^{\prime}, M^{\prime \prime}\right)=\left\{\omega \in \Omega(G) \mid \omega\left(S\left(M^{\prime \prime}\right)\right)=S\left(M^{\prime}\right)\right\} .
$$

Each connected component of

$$
\mathfrak{a}_{M^{\prime}}-\left(\bigcup_{\alpha \in R(G)-R\left(M^{\prime}\right)} \operatorname{ker} \alpha\right)
$$

is called a chamber of $\mathfrak{a}_{M^{\prime}}$. The following lemma is [Cas74, Proposition 1.2.2].

Lemma A.2. If $C$ is a chamber of $\mathfrak{a}_{M^{\prime}}$, then there exist unique $S\left(M^{\prime \prime}\right)$ and $\omega \in \Omega\left(M^{\prime}, M^{\prime \prime}\right)$ such that

$$
\omega\left(C_{M^{\prime \prime}}^{+}\right)=C
$$

Lemma A.3. Let $\alpha \in R^{+}\left(G ; \mathfrak{a}_{M}\right)$. If $\operatorname{ker} \alpha \cap \overline{C_{M}^{+}}$contains a non-empty open subset of ker $\alpha$, then there exists a simple root $\tilde{\alpha} \in S(G)$ such that

$$
\operatorname{res}_{\mathfrak{a}_{M}}(\tilde{\alpha}) \in \mathbb{R}_{+}^{\times} \alpha .
$$

Proof. Put $S\left(M^{\prime}\right)=\left\{\tilde{\beta} \in S(G) \mid \tilde{\beta}(a)=0\right.$ for all $\left.a \in C_{\alpha}\right\}$, where $C_{\alpha}=\operatorname{ker} \alpha \cap \overline{C_{M}^{+}}$. Since $C_{\alpha} \subset \overline{C_{M}^{+}}$, we have $\tilde{\beta}(a) \geqslant 0$ for all $a \in C_{\alpha}$ and $\tilde{\beta} \in S(G)$. Thus

$$
R\left(M^{\prime}\right)=\left\{\tilde{\beta} \in R(G) \mid \tilde{\beta}(a)=0 \text { for all } a \in C_{\alpha}\right\} .
$$




\section{K. HiRAGA}

Since this shows that a root $\tilde{\alpha}^{\prime}$ satisfying $\operatorname{res}_{\mathfrak{a}_{M}}\left(\tilde{\alpha}^{\prime}\right)=\alpha$ is contained in $R\left(M^{\prime}\right)$, we have $R\left(M^{\prime}\right) \supsetneq$ $R(M)$. Thus $S\left(M^{\prime}\right) \supsetneq S(M)$. Take a simple root $\tilde{\alpha} \in S\left(M^{\prime}\right)-S(M)$. Then $\tilde{\alpha}$ satisfies $\operatorname{res}_{\mathfrak{a}_{M}}(\tilde{\alpha}) \in$ $\mathbb{R}_{+}^{\times} \alpha$.

Let $C$ be a chamber of $\mathfrak{a}_{M}$ whose closure $\bar{C}$ contains a non-empty open subset of ker $\alpha$. Then Lemma 2 asserts that there exist unique $S\left(M^{\prime}\right)$ and $\omega \in \Omega\left(M, M^{\prime}\right)$ such that $\omega\left(C_{M^{\prime}}^{+}\right)=C$. By Lemma 3, we can choose a simple root $\tilde{\alpha}$ such that

$$
\operatorname{res}_{\mathfrak{a}_{M^{\prime}}}(\tilde{\alpha})=\mathbb{R}_{+}^{\times} \omega^{-1} \alpha .
$$

Since we may replace $S(M)$ by $S\left(M^{\prime}\right)$ and $\alpha$ by $\omega^{-1} \alpha$, it is enough to prove Lemma 1 under the condition $\alpha \in \operatorname{res}_{\mathfrak{a}_{M}}(S(G))$. Therefore we assume that there exists a simple root $\tilde{\alpha}$ such that $\alpha=\operatorname{res}_{\mathfrak{a}_{M}}(\tilde{\alpha})$. Put $S\left(M_{\alpha}\right)=S(M) \cup\{\tilde{\alpha}\}$. We denote by $\omega_{-}^{\alpha}$ the longest element of $\Omega\left(M_{\alpha}\right)$.

Lemma A.4. Let $\alpha$ and $\tilde{\alpha}$ be as above, then $\alpha$ is $R(G)$-symmetric if and only if

$$
\omega_{-}^{\alpha} \tilde{\alpha}=-\tilde{\alpha} .
$$

Moreover, if $\alpha$ is $R(G)$-symmetric, then we have

$$
\operatorname{res}_{\mathfrak{a}_{M}}\left(\omega_{-}^{\alpha}\right)=s_{\alpha}
$$

Proof. The condition for $\omega \in \Omega(G)$ to satisfy $\operatorname{res}_{\mathfrak{a}_{M}}(\omega)=s_{\alpha}$ is that $\omega(R(M))=R(M)$, $\omega \tilde{\alpha}<0$ and $\omega\left(R\left(M_{\alpha}\right)\right)=R\left(M_{\alpha}\right)$. It is easy to see that such an element $\omega$ exists if and only if we have $\omega_{-}^{\alpha} \tilde{\alpha}=-\tilde{\alpha}$. Moreover, if $\omega_{-}^{\alpha} \tilde{\alpha}=-\tilde{\alpha}$, then the condition for $\omega$ to satisfy $\operatorname{res}_{\mathfrak{a}_{M}}(\omega)=s_{\alpha}$ is that $\omega \in \omega_{-}^{\alpha} \cdot \Omega(M)$. So the lemma is proved.

Let $R$ be a root system and $\omega_{-}$the longest element of the Weyl group of $R$ with respect to a positive root system $R^{+}$. Then it is not hard to check that there exist mutually orthogonal roots $\tilde{\alpha}_{1}, \ldots, \tilde{\alpha}_{p} \in R^{+}$such that

$$
s_{\tilde{\alpha}_{1}} \cdots s_{\tilde{\alpha}_{p}}=\omega_{-} .
$$

Now, we prove Lemma 1. Suppose that $\alpha$ is $R(G)$-symmetric. By Lemma 4, we have

$$
\operatorname{res}_{\mathfrak{a}_{M}}\left(\omega_{-}^{\alpha}\right)=s_{\alpha}
$$

For $R=R\left(M_{\alpha}\right)$ and $\omega_{-}=\omega_{-}^{\alpha}$, take $\tilde{\alpha}_{1}, \ldots, \tilde{\alpha}_{p}$ as above. We may arrange the index so that $\tilde{\alpha}_{1}, \ldots, \tilde{\alpha}_{r}$ are not contained in $R^{+}(M)$ and that $\tilde{\alpha}_{r+1}, \ldots, \tilde{\alpha}_{p}$ are contained in $R^{+}(M)$. Then we have

$$
\begin{gathered}
\operatorname{res}_{\mathfrak{a}_{M}}\left(s_{\tilde{\alpha}_{1}} \cdots s_{\tilde{\alpha}_{r}}\right)=\operatorname{res}_{\mathfrak{a}_{M}}\left(\omega_{-}^{\alpha}\right)=s_{\alpha}, \\
\operatorname{res}_{\mathfrak{a}_{M}} \tilde{\alpha}_{1}, \ldots, \operatorname{res}_{\mathfrak{a}_{M}} \tilde{\alpha}_{r} \in \mathbb{R}_{+}^{\times} \alpha .
\end{gathered}
$$

This proves Lemma 1.

Until the end of this section, we fix $S\left(M^{H}\right), R(H)$ as in $\S 2$.

Lemma A.5 (Lemma 2.1). Let $\omega \in \tilde{D}_{M}^{-1}$ and $\omega^{\prime} \in \Omega(G)_{H}$. Let $\tilde{\alpha} \in R^{+}(G)$ be a positive root such that $\operatorname{res}_{H}(\tilde{\alpha}) \neq 0$. If $\omega \omega^{\prime} \tilde{\alpha}>0$, then for any $\tilde{\alpha}^{\prime} \in R^{+}(G)$ satisfying $\operatorname{res}_{H}\left(\tilde{\alpha}^{\prime}\right) \in \mathbb{R}_{+}^{\times} \operatorname{res}_{H}(\tilde{\alpha})$, we have $\omega \omega^{\prime} \tilde{\alpha}^{\prime}>0$ and if $\omega \omega^{\prime} \tilde{\alpha}<0$, then for any $\tilde{\alpha}^{\prime} \in R^{+}(G)$ satisfying $\operatorname{res}_{H}\left(\tilde{\alpha}^{\prime}\right) \in \mathbb{R}_{+}^{\times} \operatorname{res}_{H}(\tilde{\alpha})$, we have $\omega \omega^{\prime} \tilde{\alpha}^{\prime}<0$.

Proof. Take $a_{+} \in C_{M}^{+}$. Suppose that $\tilde{\alpha}^{\prime} \in R^{+}(G)$ satisfies $\operatorname{res}_{H}\left(\tilde{\alpha}^{\prime}\right)=c \cdot \operatorname{res}_{H}(\tilde{\alpha})$, where $c \in \mathbb{R}_{+}^{\times}$. Then we have $\left(c \cdot \tilde{\alpha}-\tilde{\alpha}^{\prime}\right)\left(\mathfrak{a}^{H}\right)=0$. By the definition of $\tilde{D}_{M}$, we have $\omega^{\prime-1} \omega^{-1}\left(\mathfrak{a}_{M}\right) \subset \mathfrak{a}^{H}$. Thus, $\omega \omega^{\prime}\left(c \cdot \tilde{\alpha}-\tilde{\alpha}^{\prime}\right)\left(\mathfrak{a}_{M}\right)=0$. Therefore,

$$
c \cdot \omega \omega^{\prime} \tilde{\alpha}\left(a_{+}\right)=\omega \omega^{\prime} \tilde{\alpha}^{\prime}\left(a_{+}\right) .
$$

Now, suppose that $\omega \omega^{\prime} \tilde{\alpha}>0$. If $\omega \omega^{\prime} \tilde{\alpha}\left(a_{+}\right)>0$, then $\omega \omega^{\prime} \tilde{\alpha}^{\prime}\left(a_{+}\right)>0$. This implies that $\omega \omega^{\prime} \tilde{\alpha}^{\prime}>0$. If $\omega \omega^{\prime} \tilde{\alpha}\left(a_{+}\right)=0$, then $\omega \omega^{\prime} \tilde{\alpha}^{\prime}\left(a_{+}\right)=0$. Thus $\omega \omega^{\prime} \tilde{\alpha}, \omega \omega^{\prime} \tilde{\alpha}^{\prime} \in R(M)$. Since $\omega \in \tilde{D}_{M}^{-1}$ and 


\section{ON FUNCTORIALITY OF ZELEVINSKI INVOLUTIONS}

since $\omega \omega^{\prime} \tilde{\alpha} \in R^{+}(M)$, we have $\omega^{\prime} \tilde{\alpha}>0$. This implies that $c^{-1} \cdot \operatorname{res}_{H}\left(\omega^{\prime} \tilde{\alpha}^{\prime}\right)=\operatorname{res}_{H}\left(\omega^{\prime} \tilde{\alpha}\right) \in R^{+}\left(G ; \mathfrak{a}^{H}\right)$. Therefore, $\omega^{\prime} \tilde{\alpha}^{\prime}>0$. Since $\omega \in \tilde{D}_{M}^{-1}$ and since $\omega \omega^{\prime} \tilde{\alpha}^{\prime} \in R(M)$, this shows that $\omega \omega^{\prime} \tilde{\alpha}^{\prime}>0$.

In the following, we prove Proposition 2.2. Obviously, we have $\omega \in \Omega(G)_{M, H}$ if and only if $\omega \Omega\left(M^{H}\right) \omega^{-1} \subset \Omega(M)$.

Lemma A.6. Let $\omega \in \Omega(G)_{M, H}$, then we have

$$
\Omega(M) \omega \Omega(H) \cap D_{M, H} \neq \emptyset .
$$

Proof. It is easy to see that $\tilde{D}_{M}^{-1}$ is a system of representatives for $\Omega(M) \backslash \Omega(G)_{M, H}$. Therefore, we may assume that $\omega \in \tilde{D}_{M}^{-1}$. Put $\tilde{R}^{+}(H)=\left\{\tilde{\alpha} \in R(G) \mid \operatorname{res}_{H}(\tilde{\alpha}) \in R^{+}(H)\right\}$. By Lemma 2.1, we can choose $\omega_{0} \in \omega \Omega(H)$ such that $\omega_{0}\left(\tilde{R}^{+}(H)\right)>0$. Now, we prove that $\Omega(M) \omega_{0} \cap D_{M, H} \neq \emptyset$ by induction on $l_{M}\left(\omega_{0}^{-1}\right)$ under the condition $\omega_{0}\left(\tilde{R}^{+}(H)\right)>0$. If $l_{M}\left(\omega_{0}^{-1}\right)=0$, then we have $\omega_{0} \in D_{M, H}$ and the lemma is proved. Suppose that $l_{M}\left(\omega_{0}^{-1}\right)>0$. Then there exists a simple root $\tilde{\alpha} \in S(M)$ such that $\omega_{0}^{-1} \tilde{\alpha}<0$. Since $\omega_{0}\left(\tilde{R}^{+}(H)\right)>0$, we have $\operatorname{res}_{H}\left(\omega_{0}^{-1} \tilde{\alpha}\right) \notin R(H)$. Therefore, $\omega_{0}\left(\tilde{R}^{+}(H)\right)>0$ implies that $s_{\tilde{\alpha}} \omega_{0}\left(\tilde{R}^{+}(H)\right)>0$. Since $l_{M}\left(\omega_{0}^{-1} s_{\tilde{\alpha}}\right)<l_{M}\left(\omega_{0}^{-1}\right)$, we have $\Omega(M) s_{\tilde{\alpha}} \omega_{0} \cap D_{M, H} \neq \emptyset$, by the inductive assumption. Because $s_{\tilde{\alpha}} \in \Omega(M)$, this shows that $\Omega(M) \omega_{0} \cap D_{M, H} \neq \emptyset$.

Proposition A.7 (Proposition 2.2). The subset $D_{M, H} \subset \Omega(G)_{M, H}$ is a system of representatives for $\Omega(M) \backslash \Omega(G)_{M, H} / \Omega(H)$.

Proof. It is enough to show that

$$
\sharp\left(\Omega(M) \omega_{0} \Omega(H) \cap D_{M, H}\right)=1
$$

for $\omega_{0} \in D_{M, H}$. Let $\omega \in \Omega(M) \omega_{0} \Omega(H) \cap D_{M, H}$, then we can write $\omega$ as $\omega=\omega_{M} \omega_{0} \omega_{H}$ with $\omega_{M} \in$ $\Omega(M)$ and $\omega_{H} \in \Omega(H)$. We claim that $\omega \in \omega_{0} \Omega(H) \Omega\left(M^{H}\right)$. We prove this by induction on $l_{M}\left(\omega_{M}^{-1}\right)$. If $l_{M}\left(\omega_{M}^{-1}\right)=0$, then $\omega_{M}=1$. Thus $\omega \in \omega_{0} \Omega(H)$. Suppose that $l_{M}\left(\omega_{M}^{-1}\right)>0$ and $l_{H}\left(\omega_{H}\right)=0$, then we have $\operatorname{res}_{H}\left(\omega_{H}\right)=1$. Thus, $\omega_{0}\left(\mathfrak{a}^{H}\right) \supset \mathfrak{a}_{M}$ implies that $\omega_{0} \omega_{H} \omega_{0}^{-1} \in \Omega(M)$. Combining this with $l_{M}\left(\omega_{0}^{-1}\right)=l_{M}\left(\omega^{-1}\right)=0$ and $\omega=\omega_{M} \cdot\left(\omega_{0} \omega_{H} \omega_{0}^{-1}\right) \cdot \omega_{0}$, we have $\omega_{M} \cdot\left(\omega_{0} \omega_{H} \omega_{0}^{-1}\right)=1$. Thus, $\omega=\omega_{0}$. Suppose that $l_{M}\left(\omega_{M}^{-1}\right)>0$ and $l_{H}\left(\omega_{H}\right)>0$. Then there exists a positive root $\alpha \in R^{+}(H)$ such that $\omega_{H} \alpha<0$. Then, by Lemma 2.1, we have $\omega_{0} \omega_{H} \tilde{\alpha}<0$ for any $\tilde{\alpha} \in R^{+}(G)$ satisfying $\operatorname{res}_{H} \tilde{\alpha} \in \mathbb{R}_{+}^{\times} \alpha$. On the other hand, since $\omega \in D_{M, H}$, we have $\omega_{M} \omega_{0} \omega_{H} \tilde{\alpha}>0$. Because $\omega_{M}$ changes the positivity of $\omega_{0} \omega_{H} \tilde{\alpha}$, we have $\omega_{0} \omega_{H} \tilde{\alpha} \in R(M)$. Put $\tilde{\alpha}^{\prime}=\omega_{H} \tilde{\alpha}$ and $\alpha^{\prime}=\omega_{H} \alpha$. Since $\operatorname{res}_{H}\left(\tilde{\alpha}^{\prime}\right)$ is $R(G)$-symmetric, we can choose $\tilde{\alpha}_{1}^{\prime}, \ldots, \tilde{\alpha}_{r}^{\prime} \in R^{+}(G)$ as in Lemma 1 . For $i=1, \ldots, r$, put $\tilde{\beta}_{i}=\omega_{0} \tilde{\alpha}_{i}^{\prime}$. Since $\operatorname{res}_{H}\left(\omega_{H}^{-1} \tilde{\alpha}_{i}^{\prime}\right) \in \mathbb{R}_{+}^{\times} \alpha$, the above argument shows that $\tilde{\beta}_{i} \in R^{-}(M)$ and that $\omega_{M} \tilde{\beta}_{i} \in R^{+}(M)$. Put $\tilde{s}_{\alpha^{\prime}}=s_{\tilde{\alpha}_{1}^{\prime}} \cdots s_{\tilde{\alpha}_{r}^{\prime}}=\omega_{0}^{-1} s_{\tilde{\beta}_{1}} \cdots s_{\tilde{\beta}_{r}} \omega_{0}$. Then we have $\tilde{s}_{\alpha^{\prime}} \in \Omega(H) \Omega\left(M^{H}\right)$ and

$$
\omega=\omega_{M} \omega_{0} \omega_{H}=\omega_{M} s_{\tilde{\beta}_{1}} \cdots s_{\tilde{\beta}_{r}} \cdot \omega_{0} \cdot \tilde{s}_{\alpha^{\prime}} \omega_{H} \cdot
$$

Since $l_{M}\left(s_{\tilde{\beta}_{r}}^{-1} \cdots s_{\tilde{\beta}_{1}}^{-1} \omega_{M}^{-1}\right)<l_{M}\left(\omega_{M}^{-1}\right)$, we have

$$
\omega \in \omega_{0} \Omega(H) \Omega\left(M^{H}\right)
$$

by the inductive assumption. Now, we can write $\omega$ as $\omega=\omega_{0} \omega_{H}^{\prime}$ with $\omega_{H}^{\prime} \in \Omega(H) \Omega\left(M^{H}\right)$. Since $l_{H}(\omega)=l_{H}\left(\omega_{0}\right)=0$, we have $\omega_{H}^{\prime} \in \Omega\left(M^{H}\right)$. Thus, $\omega_{0} \omega_{H}^{\prime} \omega_{0}^{-1} \in \Omega(M)$. Therefore, $l_{M}\left(\omega^{-1}\right)=$ $l_{M}\left(\omega_{0}^{-1}\right)=0$ shows that $\omega_{0} \omega_{H}^{\prime} \omega_{0}^{-1}=1$, since $\omega=\omega_{0} \omega_{H}^{\prime} \omega_{0}^{-1} \cdot \omega_{0}$. Hence, $\omega=\omega_{0}$.

We denote by $\mathbb{C}[\Omega(G)]$ the group $\operatorname{ring}$ of $\Omega(G)$. We define $\tilde{\xi}_{M} \in \mathbb{C}[\Omega(G)]$ by

$$
\tilde{\xi}_{M}=\sum_{\omega \in \tilde{D}_{M}} \omega
$$

In the following, we prove that

$$
\sum_{R(M) \in \mathcal{L}^{G}}(-1)^{r(M)} \tilde{\xi}_{M}=(-1)^{r\left(M^{H}\right)} \omega_{-}^{M^{H}} \omega_{-}^{G},
$$




\section{K. HiragA}

where $\omega_{-}^{G}$ is the longest element of $\Omega(G)$ and $\omega_{-}^{M^{H}}$ is the longest element of $\Omega\left(M^{H}\right)$. We have $\omega \in \tilde{D}_{M}$ if and only if $R^{+}\left(M^{H}\right) \subset \omega\left(R^{+}(M)\right) \subset R^{+}(G)$. Therefore, we have

$$
\begin{aligned}
\sum_{R(M) \in \mathcal{L}^{G}}(-1)^{r(M)} \tilde{\xi}_{M} & =\sum_{R(M) \in \mathcal{L}^{G}}(-1)^{r(M)}\left(\sum_{\substack{\omega \in \Omega(G) \\
R^{+}\left(M^{H}\right) \subset \omega\left(R^{+}(M)\right) \subset R^{+}(G)}} \omega\right) \\
& =\sum_{\omega \in \Omega(G)}\left(\sum_{\substack{R(M) \in \mathcal{L}^{G} \\
\omega^{-1}\left(R^{+}\left(M^{H}\right) \subset \subset R^{+}(M) \subset \omega^{-1}\left(R^{+}(G)\right)\right.}}(-1)^{r(M)}\right) \omega .
\end{aligned}
$$

For $\omega \in \Omega(G)$, put

$$
\begin{aligned}
& J_{\omega}=\left\{\tilde{\alpha} \in S(G) \mid \omega \tilde{\alpha}>0, \text { and } n_{\tilde{\beta} \tilde{\alpha}}=0 \text { for all } \tilde{\beta} \in S\left(M^{H}\right)\right\}, \\
& I_{\omega}=\left\{\tilde{\alpha} \in S(G) \mid n_{\tilde{\beta} \tilde{\alpha}} \neq 0 \text { for some } \tilde{\beta} \in S\left(M^{H}\right)\right\},
\end{aligned}
$$

where $n_{\tilde{\beta} \tilde{\alpha}}$ is defined by $\omega^{-1} \tilde{\beta}=\sum_{\tilde{\alpha} \in S(G)} n_{\tilde{\beta} \tilde{\alpha}} \cdot \tilde{\alpha}$.

Lemma A.8. Let $\omega \in \Omega(G)$, then we have

$$
I_{\omega}=\omega^{-1}\left(S\left(M^{H}\right)\right)
$$

if and only if we have

$$
\omega^{-1}\left(R^{+}\left(M^{H}\right)\right) \subset R^{+}(M) \subset \omega^{-1}\left(R^{+}(G)\right)
$$

for some $R(M) \in \mathcal{L}^{G}$.

Proof. Suppose that there exists $R(M) \in \mathcal{L}^{G}$ such that

$$
\omega^{-1}\left(R^{+}\left(M^{H}\right)\right) \subset R^{+}(M) \subset \omega^{-1}\left(R^{+}(G)\right) .
$$

Then by $\omega^{-1}\left(R^{+}\left(M^{H}\right)\right) \subset R^{+}(M)$, we have $I_{\omega} \subset S(M)$. Therefore, $R^{+}(M) \subset \omega^{-1}\left(R^{+}(G)\right)$ shows that $I_{\omega} \subset \omega^{-1}\left(R^{+}(G)\right)$. Thus, we have $\omega^{-1}\left(R^{+}\left(M^{H}\right)\right)>0$ and $\omega\left(I_{\omega}\right)>0$. Now, by considering the definition of $I_{\omega}$, we can easily show that $\omega^{-1}\left(S\left(M^{H}\right)\right) \subset S(G)$. Conversely, assume that $I_{\omega}=$ $\omega^{-1}\left(S\left(M^{H}\right)\right)$. Let $R\left(M_{I_{\omega}}\right)$ be the standard subroot system of $R(G)$ corresponding to $I_{\omega}$. Then we have

$$
\omega^{-1}\left(R^{+}\left(M^{H}\right)\right) \subset R^{+}\left(M_{I_{\omega}}\right) \subset \omega^{-1}\left(R^{+}(G)\right) .
$$

If $I_{\omega}=\omega^{-1}\left(S\left(M^{H}\right)\right)$, then we have $I_{\omega} \cup J_{\omega}=\{\tilde{\alpha} \in S(G) \mid \omega \tilde{\alpha}>0\}$.

Lemma A.9. If $\omega \in \Omega(G)$ satisfies $I_{\omega}=\omega^{-1}\left(S\left(M^{H}\right)\right)$, then we have

$$
\sum_{\substack{R(M) \in \mathcal{L}^{G} \\ \omega^{-1}\left(R^{+}\left(M^{H}\right)\right) \subset R^{+}(M) \subset \omega^{-1}\left(R^{+}(G)\right)}}(-1)^{r(M)}= \begin{cases}0, & \text { if } J_{\omega} \neq \emptyset, \\ (-1)^{r\left(M^{H}\right)}, & \text { if } J_{\omega}=\emptyset .\end{cases}
$$

Proof. Since $I_{\omega}$ is the set of simple roots of the smallest standard subroot system of $R(G)$ containing $\omega^{-1}\left(R^{+}\left(M^{H}\right)\right)$, the left-hand side of the formula is equal to

$$
\sum_{\substack{R(M) \in \mathcal{L}^{G} \\ \omega \subset S(M) \subset\left(I_{\omega} \cup J_{\omega}\right)}}(-1)^{r(M)}=\sum_{J \subset J_{\omega}}(-1)^{\sharp I_{\omega}}(-1)^{\sharp J} .
$$

The above argument shows that

$$
\sum_{R(M) \in \mathcal{L}^{G}}(-1)^{r(M)} \tilde{\xi}_{M}=(-1)^{r\left(M^{H}\right)} \sum \omega,
$$




\section{ON FUNCTORIALITY OF ZELEVINSKI INVOLUTIONS}

where the sum in the right-hand side runs over $\omega \in \Omega(G)$ satisfying $I_{\omega}=\omega^{-1}\left(S\left(M^{H}\right)\right)$ and $J_{\omega}=\emptyset$. We have $I_{\omega}=\omega^{-1}\left(S\left(M^{H}\right)\right)$ and $J_{\omega}=\emptyset$ if and only if $\omega\left(I_{\omega}\right)=S\left(M^{H}\right)$ and $\omega\left(S(G)-I_{\omega}\right)<0$. Now, it is easy to see that $\omega=\omega_{-}^{M^{H}} \omega_{-}^{G}$ is the only element that satisfies this condition. Thus, we have proved the following lemma.

Lemma A.10. We have

$$
\sum_{R(M) \in \mathcal{L}^{G}}(-1)^{r(M)} \tilde{\xi}_{M}=(-1)^{r\left(M^{H}\right)} \omega_{-}^{M^{H}} \omega_{-}^{G}
$$

For

$$
\xi=\sum_{\omega \in \Omega(G)} a_{\omega} \cdot \omega \in \mathbb{C}[\Omega(G)]
$$

we define $[\xi]_{H} \in \mathbb{C}\left[\Omega(G)_{H}\right]$ by

$$
[\xi]_{H}=\sum_{\omega \in \Omega(G)_{H}} a_{\omega} \cdot \omega .
$$

For $R(L) \in \mathcal{L}^{H}$, set $\tilde{R}(L)=\left\{\tilde{\alpha} \in R(G) \mid \operatorname{res}_{H}(\tilde{\alpha}) \in R(L)\right\}$ and $\tilde{R}^{+}(L)=\tilde{R}(L) \cap R^{+}\left(G ; \mathfrak{a}^{H}\right)$. We put

$$
\begin{gathered}
D_{L}=\left\{\omega \in \Omega(G) \mid \omega\left(\tilde{R}^{+}(L)\right)>0\right\}, \\
\xi_{L}=\sum_{\omega \in D_{L}} \omega .
\end{gathered}
$$

For $\omega \in \Omega(G)_{H}$, put $J_{\omega}^{H}=\{\alpha \in S(H) \mid \omega \alpha>0\}$. Then,

$$
\sum_{R(L) \in \mathcal{L}^{H}}(-1)^{r(L)}\left[\xi_{L}\right]_{H}=\sum_{\omega \in \Omega(G)_{H}} \sum_{\substack{R(L) \in \mathcal{L}^{H} \\ \omega(S(L))>0}}(-1)^{r(L)} \cdot \omega=\sum_{\omega \in \Omega(G)_{H}} \sum_{S(L) \subset J_{\omega}^{H}}(-1)^{r(L)} \cdot \omega=\sum_{\substack{\omega \in \Omega(G)_{H} \\ J_{\omega}^{H}=\emptyset}} \omega .
$$

Thus we have

$$
\sum_{R(L) \in \mathcal{L}^{H}}(-1)^{r(L)}\left[\xi_{L}\right]_{H}=\sum_{\substack{\omega \in \Omega(G)_{H} \\ \omega\left(R^{+}(H)\right)<0}} \omega
$$

Lemma A.11. We have

$$
\left[\omega_{-}^{M^{H}} \omega_{-}^{G} \xi_{H}\right]_{H}=\sum_{R(L) \in \mathcal{L}^{H}}(-1)^{r(L)}\left[\xi_{L}\right]_{H}
$$

Proof. We have

$$
\left[\omega_{-}^{G} \xi_{H}\right]_{H}=\sum_{\substack{\omega \in \Omega(G)_{H} \\ \omega\left(R^{+}(H)\right)<0}} \omega
$$

since $\omega \in \Omega(G)_{H}$ satisfies $\left(\omega_{-}^{G}\right)^{-1} \omega \in D_{H}$ if and only if $\omega\left(R^{+}(H)\right)<0$. On the other hand, the action of $\omega_{-}^{M^{H}} \in \Omega(G)_{H}$ on $\omega(R(H))$ is trivial. Hence we have

$$
\left[\omega_{-}^{M^{H}} \omega_{-}^{G} \xi_{H}\right]_{H}=\omega_{-}^{M^{H}}\left[\omega_{-}^{G} \xi_{H}\right]_{H}=\omega_{-}^{M^{H}} \cdot \sum_{\substack{\omega \in \Omega(G)_{H} \\ \omega\left(R^{+}(H)\right)<0}} \omega=\sum_{\substack{\omega \in \Omega(G)_{H} \\ \omega\left(R^{+}(H)\right)<0}} \omega .
$$

Lemma A.12 (Lemma 2.3). Let $\omega \in D_{M, H}$, then

$$
R(H) \cap \operatorname{res}_{H}\left(\omega^{-1}(R(M))\right) \in \mathcal{L}^{H} .
$$

Proof. For $\alpha_{i} \in S(H)$, fix $\tilde{\alpha}_{i} \in R^{+}(G)$ such that $\operatorname{res}_{H}\left(\tilde{\alpha}_{i}\right)=\alpha_{i}$. Let $\alpha \in R^{+}(H)$. Choose $\tilde{\alpha} \in R^{+}(G)$ such that $\operatorname{res}_{H}(\tilde{\alpha})=\alpha$. Write $\alpha=\sum_{\alpha_{i} \in S(H)} n_{i} \cdot \alpha_{i}$. Then $\operatorname{res}_{\omega\left(\mathfrak{a}^{H}\right)}(\omega \tilde{\alpha})=\sum_{\alpha_{i} \in S(H)} n_{i} \cdot \operatorname{res}_{\omega\left(\mathfrak{a}^{H}\right)}\left(\omega \tilde{\alpha}_{i}\right)$. 


\section{K. Hiraga}

Since $\omega \in D_{M, H}$, we have $\omega \alpha_{i}>0$. Thus, $\omega \tilde{\alpha}_{i}=\sum_{\tilde{\beta}_{j} \in S(G)} m_{i j} \cdot \tilde{\beta}_{j}$, where $m_{i j} \geqslant 0$. Hence, by $\omega\left(\mathfrak{a}^{H}\right) \supset \mathfrak{a}_{M}$, we have

$$
\operatorname{res}_{\mathfrak{a}_{M}}(\omega \tilde{\alpha})=\sum_{i} n_{i} \cdot \operatorname{res}_{\mathfrak{a}_{M}}\left(\omega \tilde{\alpha}_{i}\right)=\sum_{i, j} n_{i} m_{i j} \cdot \operatorname{res}_{\mathfrak{a}_{M}}\left(\tilde{\beta}_{j}\right) .
$$

Assume $\tilde{\alpha} \in \omega^{-1}(R(M))$, then we have $n_{i} m_{i j}=0$ for any $\tilde{\beta}_{j} \in S(G)-S(M)$ and $\alpha_{i} \in S(M)$. Therefore, for each $\tilde{\alpha}_{i}$ satisfying $n_{i} \neq 0$, we have $\tilde{\alpha}_{i} \in \tilde{R}(H) \cap \omega^{-1}(R(M))$. Hence, $R(H) \cap$ $\operatorname{res}_{H}\left(\omega^{-1}(R(M))\right)$ is standard.

In the following, we prove that

$$
\sum_{R(M) \in \mathcal{L}^{G}}(-1)^{r(M)} a_{M, H, L}=(-1)^{r\left(M^{H}\right)}(-1)^{r(L)} .
$$

We prove this by imitating the proof in [Car93, $\S 2.7]$. Let $\Omega(L) \subset \Omega(H)$ be the Weyl group of $R(L)$, which we regard as a subgroup of $\Omega(G)$. We put $\mathfrak{a}_{L}=\left\{a \in \mathfrak{a}^{H} \mid \alpha(a)=0\right.$ for all $\left.\alpha \in S(L)\right\}$. If $\omega \in D_{M, H, L}$, then we have

$$
\Omega(H) \cap \omega^{-1} \Omega(M) \omega=\Omega(L) .
$$

For $\alpha \in R^{+}(H)$, put $\operatorname{ext}(\alpha)=\left\{\tilde{\alpha} \in R^{+}(G) \mid \operatorname{res}_{H}(\tilde{\alpha})=\alpha\right\}$. Let $\omega \in \Omega(G)$. In the following lemma, we say that $l_{L}(\omega)$ can be defined if either $\omega(\operatorname{ext}(\alpha)) \subset R^{+}(G)$ or $\omega(\operatorname{ext}(\alpha)) \subset R^{-}(G)$ holds for each $\alpha \in R^{+}(L)$. We write $\omega \alpha>0$ if $\omega(\operatorname{ext}(\alpha)) \subset R^{+}(G)$ and $\omega \alpha<0$ if $\omega(\operatorname{ext}(\alpha)) \subset R^{-}(G)$. If $l_{L}(\omega)$ can be defined, then we put

$$
l_{L}(\omega)=\sharp\left\{\alpha \in R^{+}(L) \mid \omega \alpha<0\right\} .
$$

Since $l_{L}\left(\omega^{-1}\right)$ can be defined for any $\omega \in \Omega(H)$, we have

$$
\Omega(H) \cap D_{L}^{-1}=\left\{\omega \in \Omega(H) \mid l_{L}\left(\omega^{-1}\right)=0\right\} .
$$

Lemma A.13. For $\omega \in D_{M, H, L}$, we have

$$
\Omega(M) \omega \Omega(H)=\left\{\omega_{M} \omega \omega_{H} \mid \omega_{M} \in \Omega(M), \omega_{H} \in \Omega(H) \cap D_{L}^{-1}\right\} .
$$

Moreover, if $l_{H}\left(\omega_{M} \omega \omega_{H}\right)$ can be defined, then we have

$$
l_{H}\left(\omega_{M} \omega \omega_{H}\right) \geqslant l_{H}\left(\omega_{H}\right) .
$$

Proof. Let $\omega_{M} \in \Omega(M)$ and $\omega_{H} \in \Omega(H)$. By induction on $l_{L}\left(\omega_{H}^{-1}\right)$, we prove that

$$
\omega_{M} \omega \omega_{H} \in \Omega(M) \omega\left(\Omega(H) \cap D_{L}^{-1}\right) .
$$

If $l_{L}\left(\omega_{H}^{-1}\right)=0$, then nothing remains to be proved. Suppose that $l_{L}\left(\omega_{H}^{-1}\right)>0$. Then there exists $\alpha \in S(L)$ such that $\omega_{H}^{-1} \alpha<0$. Choose $\tilde{\alpha} \in \omega^{-1}(R(M))$ such that $\operatorname{res}_{H}(\tilde{\alpha})=\alpha$. Since $\alpha$ is $R(G)$ symmetric, we can take $\tilde{\alpha}_{1}, \ldots, \tilde{\alpha}_{r}$ as in Lemma 1 . Then for $i=1, \ldots, r$, we have $\operatorname{res}_{\omega^{-1}\left(\mathfrak{a}_{M}\right)}\left(\tilde{\alpha}_{i}\right) \in$ $\mathbb{R}_{+}^{\times} \operatorname{res}_{\omega^{-1}\left(\mathfrak{a}_{M}\right)}(\tilde{\alpha})$, since $\mathfrak{a}^{H} \supset \omega^{-1}\left(\mathfrak{a}_{M}\right)$. This implies that $\tilde{\alpha}_{i}\left(\omega^{-1}\left(\mathfrak{a}_{M}\right)\right)=\tilde{\alpha}\left(\omega^{-1}\left(\mathfrak{a}_{M}\right)\right)=0$. Thus $\tilde{\alpha}_{i} \in \omega^{-1}(R(M))$. Put $\tilde{s}_{\alpha}=s_{\tilde{\alpha}_{1}} \cdots s_{\tilde{\alpha}_{r}}$. Then we have $\omega \cdot \tilde{s}_{\alpha}=s_{\omega \tilde{\alpha}_{1}} \cdots s_{\omega \tilde{\alpha}_{r}} \cdot \omega$. Let $\omega^{\prime}=\tilde{s}_{\alpha} s_{\alpha}^{-1} \in$ $\Omega\left(M^{H}\right)$, then

$$
\omega_{M} \omega \omega_{H}=\omega_{M} \omega \tilde{s}_{\alpha} \tilde{s}_{\alpha} \omega_{H}=\omega_{M} s_{\omega \tilde{\alpha}_{1}} \cdots s_{\omega \tilde{\alpha}_{r}} \cdot \omega \omega^{\prime} \omega^{-1} \cdot \omega \cdot s_{\alpha} \omega_{H} .
$$

Since $l_{L}\left(\omega_{H}^{-1} s_{\alpha}^{-1}\right)=l_{L}\left(\omega_{H}^{-1}\right)-1$ and since $s_{\omega \tilde{\alpha}_{1}} \cdots s_{\omega \tilde{\alpha}_{r}}$ and $\omega \omega^{\prime} \omega^{-1}$ are contained in $\Omega(M)$, this shows that $\omega_{M} \omega \omega_{H} \in \Omega(M) \omega\left(\Omega(H) \cap D_{L}^{-1}\right)$ by the inductive assumption.

Next, suppose that $l_{H}\left(\omega_{M} \omega \omega_{H}\right)$ can be defined. Since $\omega_{H} \in \Omega(H) \cap D_{L}^{-1}$, we have

$$
l_{H}\left(\omega_{H}\right)=\left\{\alpha \in R^{+}(H)-\omega_{H}^{-1}\left(R^{+}(L)\right) \mid \omega_{H} \alpha<0\right\}
$$

and

$$
\omega_{H}\left(R^{+}(H) \cap \omega_{H}^{-1} \operatorname{res}_{H}\left(\omega^{-1}(R(M))\right)\right)=R^{+}(L) .
$$




\section{ON FUNCTORIALITY OF ZELEVINSKI INVOLUTIONS}

On the other hand, let $\alpha \in R^{+}(H)-\operatorname{res}_{H}\left(\omega^{-1}(R(M))\right)$. Then we have $\omega \alpha>0$. Since $\omega \alpha \notin$ $\operatorname{res}_{\omega\left(\mathfrak{a}^{H}\right)}(R(M))$, we have $\omega \tilde{\alpha} \notin R(M)$ for any $\tilde{\alpha} \in R(G)$ satisfying $\operatorname{res}_{H}(\tilde{\alpha})=\alpha$. This implies that $\omega_{M} \omega \alpha>0$, since $\omega_{M} \in \Omega(M)$. Therefore, we have shown that if $\alpha \in \omega_{H}\left(R^{+}(H)-\omega_{H}^{-1}\left(R^{+}(L)\right)\right)=$ $\omega_{H}\left(R^{+}(H)-\omega_{H}^{-1} \operatorname{res}_{H}\left(\omega^{-1}(R(M))\right)\right)$ is positive, then $\omega_{M} \omega \alpha$ is positive and if it is negative, then $\omega_{M} \omega \alpha$ is negative. Thus we have proved that

$$
l_{H}\left(\omega_{M} \omega \omega_{H}\right)=l_{H}\left(\omega_{H}\right)+\sharp\left\{\alpha \in R^{+}(L) \mid \omega_{M} \omega \alpha<0\right\} .
$$

Corollary A.14. If $\omega \in D_{M, H}$, then

$$
D_{H} \cap \Omega(M) \omega \Omega(H) \subset \Omega(M) \omega .
$$

Proof. Let $\omega \in D_{M, H, L}$ and $\omega_{M} \omega \omega_{H} \in D_{H} \cap \Omega(M) \omega \Omega(H)$, where $\omega_{M} \in \Omega(M)$ and $\omega_{H} \in \Omega(H) \cap$ $D_{L}^{-1}$. Since $\omega_{M} \omega \omega_{H} \in D_{H}, l_{H}\left(\omega_{M} \omega \omega_{H}\right)$ can be defined. Moreover, we have

$$
0=l_{H}\left(\omega_{M} \omega \omega_{H}\right) \geqslant l_{H}\left(\omega_{H}\right) .
$$

Thus, $l_{H}\left(\omega_{H}\right)=0$. Since $\omega_{H} \in \Omega(H)$, this shows that $\omega_{H}=1$.

Let $x \in \Omega(G)_{H}$ and $\omega \in D_{M, H}$. Since $x \omega^{-1} \in \Omega(G)_{M, H}^{-1}$, we can write $x \omega^{-1}$ as $x \omega^{-1}=d_{M} \omega_{M}$ with $d_{M} \in \tilde{D}_{M}$ and $\omega_{M} \in \Omega(M)$. Then $d_{M}$ and $\omega_{M}$ are uniquely determined by $x$ and $\omega$.

Lemma A.15. We have

$$
\tilde{D}_{M}^{-1} x \cap D_{H} \cap \Omega(M) \omega \Omega(H)= \begin{cases}\left\{d_{M}^{-1} x\right\}, & \text { if } d_{M}^{-1} x \in D_{H}, \\ \emptyset, & \text { otherwise. }\end{cases}
$$

Proof. Suppose that $d \in \tilde{D}_{M}$ satisfies $d^{-1} x \in D_{H} \cap \Omega(M) \omega \Omega(H)$. Then Corollary 14 asserts that there exists $\omega_{M}^{\prime} \in \Omega(M)$ such that $d^{-1} x=\omega_{M}^{\prime} \omega$. This implies that $x \omega^{-1}=d \omega_{M}^{\prime}$ and shows that $d=d_{M}$. Therefore, $\tilde{D}_{M}^{-1} x \cap D_{H} \cap \Omega(M) \omega \Omega(H)$ is equal to $\left\{d_{M}^{-1} x\right\}$ or $\emptyset$. Since $d_{M}^{-1} x=\omega_{M} \omega$, the element $d_{M}^{-1} x$ is contained in $\tilde{D}_{M}^{-1} x \cap D_{H} \cap \Omega(M) \omega \Omega(H)$ if and only if $d_{M}^{-1} x \in D_{H}$.

Put

$$
R(L)=R(H) \cap \operatorname{res}_{H}\left(\omega^{-1}(R(M))\right) .
$$

Then $\omega \in D_{M, H, L}$.

Lemma A.16. We have $d_{M}^{-1} x \in D_{H}$ if and only if $x \in D_{L}$.

Proof. Since $\mathfrak{a}^{H} \supset \omega^{-1}\left(\mathfrak{a}_{M}\right)$, we have $\tilde{R}^{+}(L)=\tilde{R}^{+}(H) \cap \omega^{-1}(R(M))$. Because $d_{M}^{-1} x \omega^{-1}=\omega_{M}$ preserves $R(M)$, this implies that

$$
d_{M}^{-1} x\left(\tilde{R}^{+}(L)\right)=d_{M}^{-1} x\left(\tilde{R}^{+}(H)\right) \cap R(M) .
$$

Now, suppose $d_{M}^{-1} x \in D_{H}$, then we have $d_{M}^{-1} x\left(\tilde{R}^{+}(L)\right) \subset R^{+}(M)$. Therefore, $x\left(\tilde{R}^{+}(L)\right) \subset R^{+}(G)$. Conversely, suppose that $x\left(\tilde{R}^{+}(L)\right) \subset R^{+}(G)$. Then we have $d_{M}^{-1} x\left(\tilde{R}^{+}(L)\right) \subset R^{+}(G)$. Now, we consider $d_{M}^{-1} x\left(\tilde{R}^{+}(H)-\tilde{R}^{+}(L)\right)$. Since $d_{M}^{-1} x=\omega_{M} \omega$, this is equal to $\omega_{M} \omega\left(\tilde{R}^{+}(H)-\tilde{R}^{+}(L)\right)$. Because $\omega \in D_{M, H, L}$, we have $\omega\left(\tilde{R}^{+}(H)-\tilde{R}^{+}(L)\right) \subset R^{+}(G)-R^{+}(M)$. Since $\omega_{M}$ preserves the positivities of the roots in $R^{+}(G)-R^{+}(M)$, this shows that $d_{M}^{-1} x\left(\tilde{R}^{+}(H)-\tilde{R}^{+}(L)\right) \subset R^{+}(G)$. Thus, $d_{M}^{-1} x \in$ $D_{H}$.

Lemma A.17. Let $x \in \Omega(G)_{H}$, then we have

$$
\sharp\left(\tilde{D}_{M}^{-1} x \cap D_{H}\right)=\sum a_{M, H, L},
$$

where the sum in the right-hand side runs over $R(L) \in \mathcal{L}^{H}$ satisfying $x \in D_{L}$. 


\section{K. HiRAGA}

Proof. If $d \in \tilde{D}_{M}$, then $d^{-1} x \in \Omega(G)_{M, H}$. Therefore, Proposition 2.2 asserts that there exists a unique $\omega \in D_{M, H}$ such that $d^{-1} x \in \Omega(M) \omega \Omega(H)$. Hence, by Lemma 15, we have

$$
\sharp\left(\tilde{D}_{M}^{-1} x \cap D_{H}\right)=\sharp\left\{\omega \in D_{M, H} \mid \tilde{D}_{M}^{-1} x \cap D_{H} \cap \Omega(M) \omega \Omega(H) \neq \emptyset\right\} .
$$

Since $D_{M, H}=\bigcup_{R(L) \in \mathcal{L}^{H}} D_{M, H, L}$, the required equation follows immediately from Lemma 16 .

Proposition A.18. We have

$$
\left[\tilde{\xi}_{M} \xi_{H}\right]_{H}=\sum_{R(L) \in \mathcal{L}^{H}} a_{M, H, L}\left[\xi_{L}\right]_{H}
$$

Proof. Let $x \in \Omega(G)_{H}$, then the coefficient of $x$ in $\left[\tilde{\xi}_{M} \xi_{H}\right]_{H}$ is equal to the number of the pair $\left(d_{M}, \omega_{H}\right) \in \tilde{D}_{M} \times D_{H}$ satisfying $x=d_{M} \omega_{H}$. Therefore, the coefficient of $x$ in $\left[\tilde{\xi}_{M} \xi_{H}\right]_{H}$ is $\sharp\left(\tilde{D}_{M}^{-1} x \cap\right.$ $D_{H}$ ), and Lemma 17 asserts that this is equal to the coefficient of $x$ in the right-hand side of the formula.

Theorem A.19 (Theorem 2.4). We have

$$
\sum_{R(M) \in \mathcal{L}^{G}}(-1)^{r(M)} a_{M, H, L}=(-1)^{r\left(M^{H}\right)} \cdot(-1)^{r(L)} .
$$

Proof. By Lemma 10, we have

$$
\omega_{-}^{M^{H}} \omega_{-}^{G}=(-1)^{r\left(M^{H}\right)} \sum_{R(M) \in \mathcal{L}^{G}}(-1)^{r(M)} \tilde{\xi}_{M} .
$$

Therefore, by using Lemma 11 and Proposition 18, we have

$$
\begin{aligned}
\sum_{R(L) \in \mathcal{L}^{H}}(-1)^{r(L)}\left[\xi_{L}\right]_{H} & =\left[\omega_{-}^{M^{H}} \omega_{-}^{G} \xi_{H}\right]_{H} \\
& =(-1)^{r\left(M^{H}\right)} \sum_{R(M) \in \mathcal{L}^{G}}(-1)^{r(M)}\left[\tilde{\xi}_{M} \xi_{H}\right]_{H} \\
& =(-1)^{r\left(M^{H}\right)} \sum_{R(M) \in \mathcal{L}^{G}}(-1)^{r(M)} \sum_{R(L) \in \mathcal{L}^{H}} a_{M, H, L}\left[\xi_{L}\right]_{H} .
\end{aligned}
$$

It is easy to see that $\left[\xi_{L}\right]_{H}$ are linearly independent. Hence, by comparing the coefficient of $\left[\xi_{L}\right]_{H}$, we get the required formula.

For $R(M), R\left(M^{\prime}\right) \in \mathcal{L}^{G}$, we put

$$
D_{M, M^{\prime}}=\left\{\omega \in \Omega(G) \mid l_{M}\left(\omega^{-1}\right)=l_{M^{\prime}}(\omega)=0\right\} .
$$

Then $D_{M, M^{\prime}}$ is a set of representatives for $\Omega(M) \backslash \Omega(G) / \Omega\left(M^{\prime}\right)$.

Lemma A.20 (Lemma 2.5). Let $R(M), R\left(M_{0}\right) \in \mathcal{L}^{G}$. Assume that $R\left(M_{0}\right)$ has no other associate standard subroot system than $R\left(M_{0}\right)$ itself. If $R(M)$ satisfies $\omega\left(\mathfrak{a}_{M}\right) \subset \mathfrak{a}_{M_{0}}$ for some $\omega \in \Omega(G)$, then we have

$$
R(M) \supset R\left(M_{0}\right) .
$$

Proof. Choose a chamber $C$ of $\mathfrak{a}_{M_{0}}$ such that the closure $\bar{C}$ of $C$ contains a non-empty open subset of $\omega\left(\mathfrak{a}_{M}\right)$. By Lemma 2 we can take $\omega^{\prime} \in \Omega(G)_{\mathfrak{a}_{M_{0}}}$ such that $\omega^{\prime}(C)=C_{M_{0}}^{+}$. Then $\omega^{\prime} \omega\left(\mathfrak{a}_{M}\right)$ is a standard subspace and there exists $S\left(M^{\prime}\right) \subset S(G)$ such that $\omega^{\prime} \omega(R(M))=R\left(M^{\prime}\right)$. Take $\omega_{0} \in$ $D_{M, M^{\prime}} \cap \Omega(M) \omega^{-1} \omega^{\prime-1} \Omega\left(M^{\prime}\right)$. Since $\omega_{0}\left(R\left(M^{\prime}\right)\right)=R(M)$, we have $\omega_{0}\left(S\left(M^{\prime}\right)\right)=S(M)$. Therefore,

$$
\omega_{0}\left(S\left(M_{0}\right)\right) \subset \omega_{0}\left(S\left(M^{\prime}\right)\right)=S(M) \subset S(G) .
$$

This implies that $\omega_{0}\left(R\left(M_{0}\right)\right)$ is an associate standard subroot system of $R\left(M_{0}\right)$. Hence $R\left(M_{0}\right)=$ $\omega_{0}\left(R\left(M_{0}\right)\right) \subset R(M)$. 


\section{ON FUNCTORIALITY OF ZELEVINSKI INVOLUTIONS}

\section{ACKNowledgements}

The author would like to express his gratitude to Professor Tamotsu Ikeda and Professor Takuya Konno. Conjecture 1.2 has arisen from discussions with them. The involution $d$ on the Arthur parameters was suggested by Takuya Konno. The author also expresses his gratitude to Professor Takuya Konno and Doctor Kazuko Konno for informing the author of their results. The author also expresses his gratitude to Professor Tamotsu Ikeda for his advice. Finally the author would like to express his gratitude to Professor Hiroyuki Yoshida for his advice and encouragement.

\section{REFERENCES}

Art89 J. Arthur, Unipotent automorphic representations: conjectures. Orbites unipotentes et représentations, II, Astérisque 171-172 (1989), 13-71.

Art93 J. Arthur, On elliptic tempered characters, Acta Math. 171 (1993), 73-138.

Art96 J. Arthur, On local character relations, Selecta Math. (N.S.) 2 (1996), 501-579.

Aub95 A.-M. Aubert, Dualité dans le groupe de Grothendieck de la catégorie des représentations lisses de longueur finie d'un groupe réductif p-adique, Trans. Amer. Math. Soc. 347 (1995), 2179-2189.

Aub96 A.-M. Aubert, Erratum: Duality in the Grothendieck group of the category of finite-length smooth representations of a p-adic reductive group, Trans. Amer. Math. Soc. 348 (1996), 4687-4690.

BZ77 I. N. Bernstein and A. V. Zelevinsky, Induced representations of reductive p-adic groups. I, Ann. Sci. École Norm. Sup. (4) 10 (1977), 441-472.

Car93 R. W. Carter, Finite groups of Lie type. Conjugacy classes and complex characters (Wiley, Chichester, 1993).

Cas74 W. Casselman, Introduction to the theory of admissible representations of p-adic reductive groups, Preprint (1974).

Cas77 W. Casselman, Characters and Jacquet modules, Math. Ann. 230 (1977), 101-105.

Har78 Harish-Chandra, Admissible invariant distributions on reductive p-adic groups, in Lie theories and their applications, Queen's Papers in Pure Appl. Math. 48 (1978), 281-347.

Kat93 S. Kato, Duality for representations of a Hecke algebra, Proc. Amer. Math. Soc. 119 (1993), 941-946.

Kon03 T. Konno and K. Konno, CAP automorphic representations of $U_{E / F}(4)$, I. Local A-packets, Preprint (2003).

KS99 R. E. Kottwitz and D. Shelstad, Foundations of twisted endoscopy, Astérisque 255 (1999).

Kot82 R. E. Kottwitz, Rational conjugacy classes in reductive groups, Duke Math. J. 49 (1982), 785-806.

Kot84 R. E. Kottwitz, Stable trace formula: cuspidal tempered terms, Duke Math. J. 51 (1984), 611-650.

Kot86 R. E. Kottwitz, Stable trace formula: elliptic singular terms, Math. Ann. 275 (1986), 365-399.

LL79 J.-P. Labesse and R. P. Langlands, L-indistinguishability for SL(2), Canad. J. Math. 31 (1979), $726-785$.

LS87 R. P. Langlands and D. Shelstad, On the definition of transfer factors, Math. Ann. 278 (1987), 219-271.

LS90 R. P. Langlands and D. Shelstad, Descent for transfer factors. The Grothendieck Festschrift, Vol. II, Progr. Math. 87 (1990), 485-563.

Lan79 R. P. Langlands, Stable conjugacy: definitions and lemmas, Canad. J. Math. 31 (1979), 700-725.

Lan83 R. P. Langlands, Les débuts d'une formule des traces stable, Publications mathématiques de l'Université Paris VII, vol. 13 (1983).

MW86 C. Moeglin and J.-L. Waldspurger, Sur l'involution de Zelevinski, J. reine angew. Math. 372 (1986), $136-177$.

Vog93 D. Vogan, The local Langlands conjecture, in Representation theory of groups and algebras, Contemp. Math. 145 (1993), 305-379. 


\section{ON FUNCTORIALITY OF ZELEVINSKI INVOLUTIONS}

Wal97 J.-L. Waldspurger, Le lemme fondamental implique le transfert, Compositio Math. 105 (1997), 153-236.

Zel80 A. V. Zelevinsky, Induced representations of reductive p-adic groups. II. On irreducible representations of $G L(n)$, Ann. Sci. École Norm. Sup. (4) 13 (1980), 165-210.

Zel81 A. V. Zelevinskiı, The p-adic analogue of the Kazhdan-Lusztig conjecture, Funktsional. Anal. i Prilozhen. 15 (1981), no. 2, 9-21, 96.

Kaoru Hiraga hiraga@math.kyoto-u.ac.jp

Department of Mathematics, Faculty of Science, Kyoto University, Kyoto 606-8502, Japan 